Robotics and Process Systems Division

\title{
BASIS FOR SELECTION OF A RESIDUAL WASTE RETRIEVAL SYSTEM FOR GUNITE AND ASSOCIATED TANK W-9 AT THE OAK RIDGE NATIONAL LABORATORY
}

\author{
B. E. Lewis \\ P. D. Lloyd \\ S. M. Killough \\ R. F. Lind \\ D. E. Rice
}

Oak Ridge National Laboratory

P.O. Box 2008

Oak Ridge, Tennessee 37831

M. A. Johnson

Tetra-Tech Inc.

800 Oak Ridge Turnpike

Oak Ridge, Tennessee 37830

O. D. Mullen

Pacific Northwest National Laboratory

Richland, Washington 99352

September 2000

Prepared by the

OAK RIDGE NATIONAL LABORATORY

Oak Ridge, Tennessee 37831

managed by

UT-BATTELLE, LLC

for the

U.S. DEPARTMENT OF ENERGY

under contract DE-AC05-OR22725

\footnotetext{
"The submitted manuscript has been authored by a contractor of the U.S. Government under contract No. DE-AC05-

OR22725. Accordingly, the U.S. Government retains a paid-

up, nonexclusive, irrevocable, worldwide license to publish or

reproduce the published form of this contribution, prepare

derivative works, distribute copies to the public, and perform

publicly and display publicly, or allow others to do so, for U.S.

Government purposes."
} 


\section{CONTENTS}

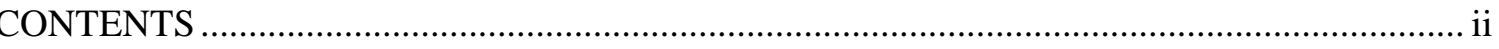

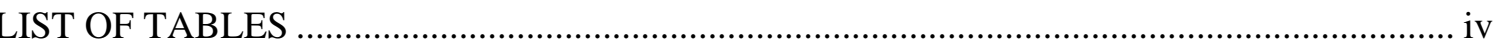

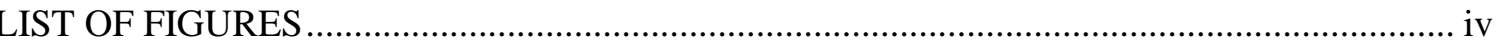

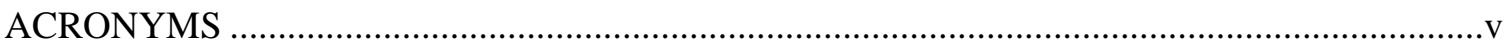

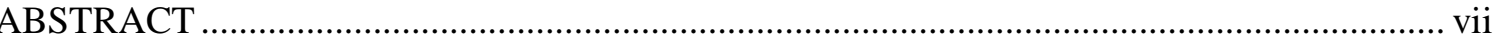

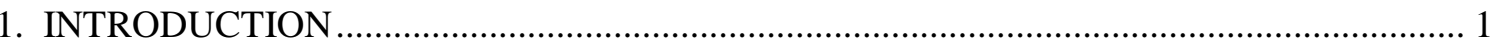

2. WASTE-CONSOLIDATION TANK DESCRIPTION AND WASTE-

CHARACTERIZATION DATA............................................................................ 10

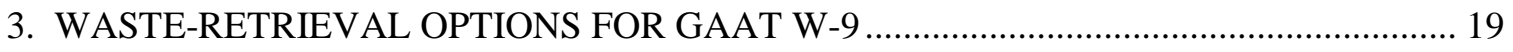

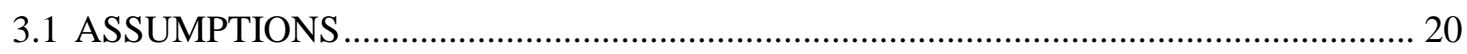

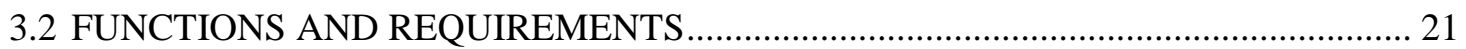

3.3 DESCRIPTION OF GAAT W-9 WASTE-RETRIEVAL AND -TRANSFER

SYSTEM OPTIONS FOR PUMPABLE WASTES ................................................... 21

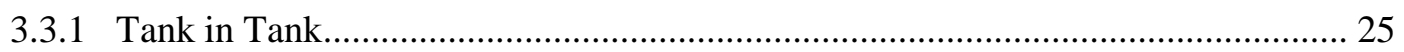

3.3.2 Additional Mixers (e.g., Russian Pulsating Mixer, Flygt, PulsAir) ................... 25

3.3.3 Waste Container/Cask and Truck Transport to MVSTs ........................................25

3.3.4 $\quad$ ESG TracPump for Retrieving and Transferring Waste to the BVESTs.............. 26

3.3.5 Pit Hog for Retrieving and Transferring Waste to the BVESTs....................... 26

3.3.6 ESG Centrifugal Pump with Grade-Level Transfer Line ................................ 26

3.3.7 Tank Waste-Retrieval System to Transfer Waste From W-9 to the

BVESTs and an AEAT Pulsed Jet Fluidic Mixing Technology and

Existing Transfer Pumps to Transfer the Waste to the MVSTs ..........................2 27

3.3.7.1 TWRS with Two Jet Pumps and Overhead Transfer Line for

Gravity Feed to the BVEST........................................................ 28

3.3.7.2 TWRS with Two Jet Pumps, SCS, and Overhead Transfer Line

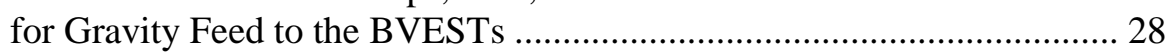

3.3.7.3 TWRS with One Jet Pump, Slurry Consolidation Tank,

DiscFlo Pump, SCS, and Overhead Transfer Line for Gravity

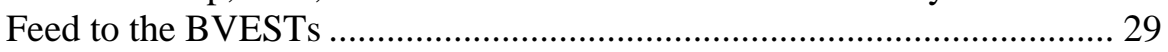

3.3.7.4 TWRS with One Jet Pump, Slurry Consolidation Tank,

DiscFlo Pump, SCS, and Grade-Level Transfer Line to the

BVESTs ................................................................................. 29

3.3.7.5 TWRS with One Jet Pump, Slurry Consolidation Tank, Booster

Pump, SCS, and Grade Level Transfer Line to the BVESTs ................... 30

3.4 DESCRIPTION OF GAAT W-9 WASTE-RETRIEVAL AND -TRANSFER SYSTEM

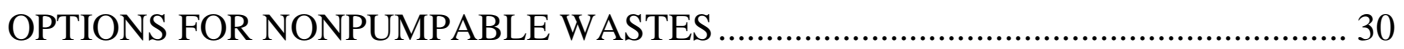

3.4 .1 RPS

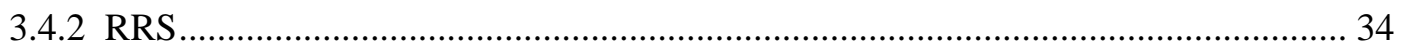

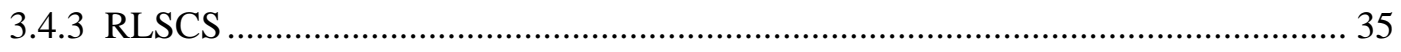

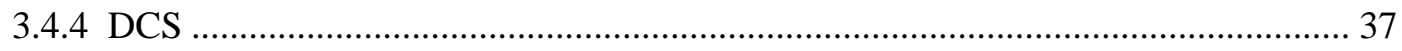

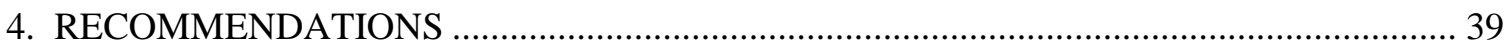




\section{CONTENTS (continued)}

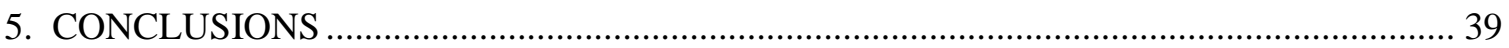

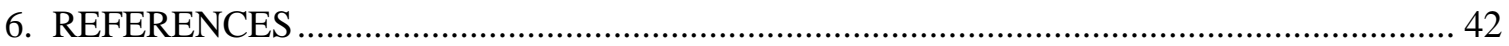

Appendix 1. ANALYSIS PLAN FOR GAAT W-9 CORE SAMPLES .................................... 44

Appendix 2. SAMPLE ANALYSIS DATA SUMMARY FOR ALL GAAT W-9

SLUDGE SAMPLES 


\section{LIST OF TABLES}

Table 1. Characteristics of the original wet sludge present in GAAT W-9 prior to consolidation .....

Table 2. Dissolution data for W-9 consolidated sludge sample 990825-014 .............................. 13

Table 3. Dissolution data for W-9 consolidated sludge solids sample 990825-015..................... 14

Table 4. Sample analysis data summary for GAAT W-9 transfers to the MVSTs....................... 15

Table 5. Sample analysis data collected during transfer operations from GAAT W-9

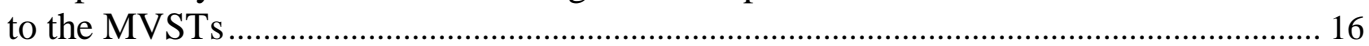

Table 6. Advantages and disadvantages of the primary waste-retrieval and transfer bptions for pumpable wastes.................................................................................. 23

Table 7. Advantages and disadvantages of the alternatives for the down selected waste retrieval and transfer option for GAAT W-9 pumpable wastes ....................................... 24

Table 8. Advantages and disadvantages of the options considered for nonpumpable

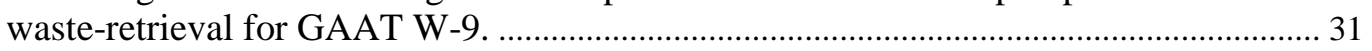

\section{LIST OF FIGURES}

Fig. 1. MLDUA holding the CSEE and operating in conjunction with the

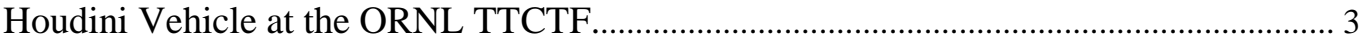

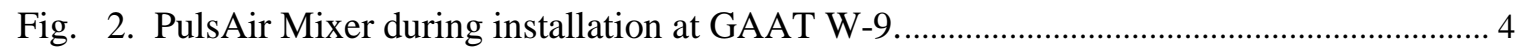

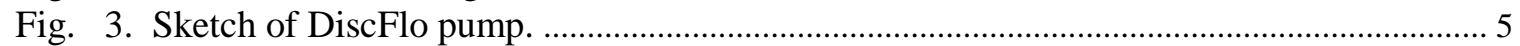

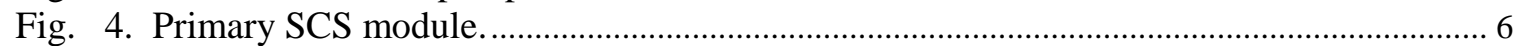

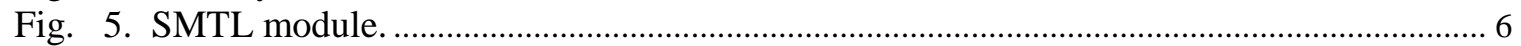

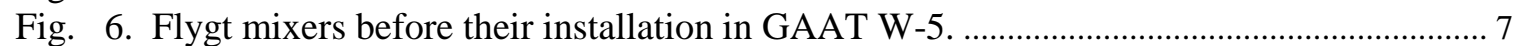

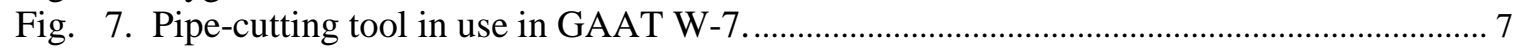

Fig. 8. Sequence of photographs of pipe-plugging tool in use inside GAAT W-6.................... 8

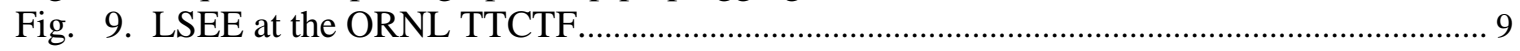

Fig. 10. W-9 supernatant and sludge samples (A) before acid digestion and

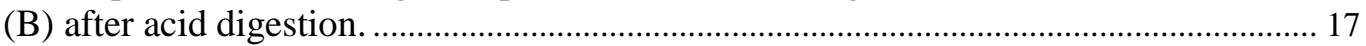

Fig. 11. W-9 supernatant and sludge samples (A) after acid digestion and settling and (B) after centrifugation. .................................................................................. 18

Fig. 12. Comparison of LasenTec and analytical data on the sludge samples from

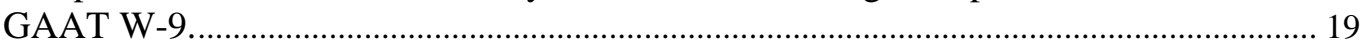

Fig. 13. Location of GAATs STF relative to the BVESTs................................................... 20

Fig. 14. Floor-cleaning end-effector by PNNL. ................................................................ 32

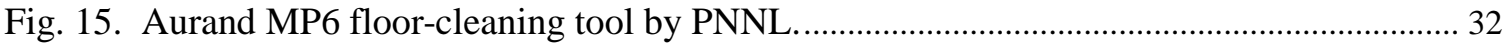

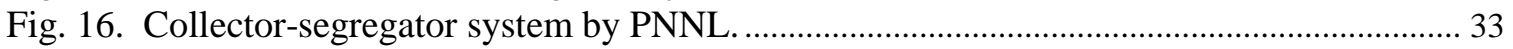

Fig. 17. Deployment and drum-handling system by PNNL ................................................. 34

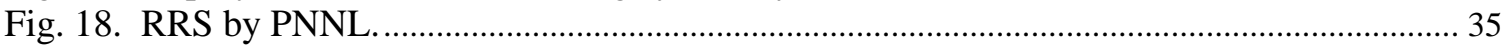

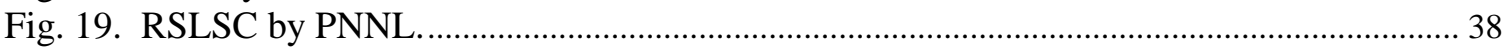

Fig. 20. WaRTS for GAAT W-9 at the ORNL TTCTF...................................................... 40

Fig. 21. Flow diagram for the residual waste-retrieval system for GAAT W-9 ......................... 41 


\section{ACRONYMS}

AEAT Atomic Energy Authority Technology

BVEST Bethel Valley Evaporator Service Tank

CSEE Confined Sluicing End-Effector

DCS Debris Consolidation System

D\&D Deactivation \& decommissioning

D.F. $\quad$ Degrees of freedom

DOE U.S. Department of Energy

ESG Environmental Specialties Group

FCE-CB Flow Control Equipment-Containment Box

FY Fiscal year

GAAT Gunite ${ }^{\mathrm{TM}}$ and Associated Tank

G-alpha Gross alpha radioactivity

G-beta Gross beta radioactivity

GSEE Gunite Scarifying End-Effector

HEPA High Efficiency Particulate Air

HMA Hose Management Arm

LLLW Liquid Low Level Waste

LSEE Linear Scarifying End-Effector

MLDUA Modified Light-Duty Utility Arm

MVST Melton Valley Storage Tank

$\mathrm{N} \quad$ North

ND No Data

NE Northeast

NTF North tank farm

OHF Old Hydrofracture Facility

ORNL Oak Ridge National Laboratory

ORO Oak Ridge Operations

OU Operable Unit

PCB Polychlorinated biphenyl

PCS Primary Conditioning System

PNNL Pacific Northwest National Laboratory

RCRA Resource Conservation and Recovery Act

RLSCS Residual Liquid and Solids Consolidation System

RPS Retrieval and Packaging System

RRS Retrieval and Reduction System 


\section{ACRONYMS (continued)}

$\begin{array}{ll}\text { SCS } & \text { Sludge Conditioning System } \\ \text { SMTL } & \text { Slurry Monitoring Test Loop } \\ \text { STF } & \text { South tank farm } \\ & \\ \text { TDS } & \text { Total dissolved solids } \\ \text { TIC } & \text { Total inorganic carbon } \\ \text { TOC } & \text { Total organic carbon } \\ \text { TRU } & \text { Transuranic } \\ \text { TS } & \text { Total solids } \\ \text { TSS } & \text { Total suspended solids } \\ \text { TSS50 } & \text { Total suspended solids greater than } 50 \mu \text { m diameter } \\ \text { TSS100 } & \text { Total suspended solids greater than } 100 \mu \text { m diameter } \\ \text { TTCTF } & \text { Tanks Technology Cold Test Facility } \\ \text { TWRS } & \text { Tank Waste Retrieval System } \\ & \\ \text { WAG } & \text { Waste Area Grouping } \\ \text { WAC } & \text { Waste Acceptance Criteria } \\ \text { WaRTS } & \text { Waste Retrieval and Transfer System } \\ \text { WD\&CS } & \text { Waste Dislodging and Conveyance System }\end{array}$




\begin{abstract}
Waste retrieval and transfer operations at the Gunite ${ }^{\mathrm{TM}}$ and Associated Tanks (GAATs) Remediation Project have been successfully accomplished using the Tank Waste Retrieval System. This system is composed of the Modified Light-Duty Utility Arm, Houdini Vehicle, Waste Dislodging and Conveyance System, Hose Management Arm, and Sludge Conditioning System. GAAT W-9 has been used as a waste-consolidation and batch-transfer tank during the retrieval of sludges and supernatants from the seven Gunite tanks in the North and South tank farms at Oak Ridge National Laboratory. tank W-9 was used as a staging tank for the transfers to the Melton Valley Storage Tanks (MVSTs). A total of 18 waste transfers from W-9 occurred between May 25, 1999, and March 30, 2000. Most of these transfers were accomplished using the PulsAir Mixer to mobilize and mix the slurry and a submersible retrieval-transfer pump to transfer the slurry through the Sludge Conditioning System and the $~ 1-m i l e ~ l o n g, ~ 2-i n .-d i a m$ waste-transfer line to the MVSTs. The transfers from W-9 have consisted of low-solids-content slurries with solids contents ranging from $\sim 2.8$ to $6.8 \mathrm{mg} / \mathrm{L}$. Of the initial $\sim 88,000$ gal of wet sludge estimated in the GAATs, a total of $\sim 60,451$ gal have been transferred to the MVSTs via tank W-9 as of March 30, 2000. Once the waste-consolidation operations and transfers from W-9 to the MVSTs are completed, the remaining material in W-9 will be mobilized and transferred to the active waste system, Bethel Valley Evaporator Service Tank W-23. Tank W-23 will serve as a batch tank for the final waste transfers from tank W-9 to the MVSTs. This report provides a summary of the requirements and recommendations for the final waste retrieval system for tank W-9, a compilation of the sample analysis data for the sludge in W-9, and brief descriptions of the various waste-retrieval system concepts that were considered for this task. The recommended residual waste retrieval system for cleanout of tank W-9 consists primarily of the existing Tank Waste Retrieval System, which, is used in conjunction with a small surge vessel placed in one of the tank risers and a positive displacement pump installed inside the Primary Conditioning System containment box. Final cleanout of tank W-9 was initiated in July and successfully completed in September 2000. The performance of the selected residual waste retrieval system will be described in a follow-on report.
\end{abstract}




\section{INTRODUCTION}

The Gunite and Associated Tanks (GAATs) are located at the Oak Ridge National Laboratory (ORNL) and are a part of Waste Area Grouping (WAG) 1. These tanks were constructed between 1943 and 1951 and were designed to contain the radioactive and chemical wastes generated by ORNL operations. A total of 12 Gunite tanks and 4 stainless-steel tanks (W-1A, $\mathrm{W}-13, \mathrm{~W}-14$, and W-15) were located in the GAATs Operable Unit (OU), which is situated in a high-traffic area in the middle of the ORNL site. About $90 \%$ of the sludge initially present in the 6 large Gunite tanks (W-5, W-6, W-7, W-8, W-9, and W-10) was retrieved during an 18-month sluicing operation from 1982 through January $1984 .{ }^{1}$ At the end of the sluicing operations in 1984, 88,000 gal of difficult-to-retrieve wet sludge remained in GAATs W-3, W-4, W-5, W-6, W-7, W-8, W-9, and W-10. Beginning in 1994, the GAATs Remediation Project began to focus on the retrieval of the remaining wastes from these tanks. Early on, it was decided that tank W-9 would be the waste-consolidation tank for the batch transfers to the Melton Valley Storage Tanks (MVSTs). Tank W-9 was used to contain the waste retrieved from the other GAATs and served as a batch-processing tank for the transfers to the MVSTs. The Sludge-Conditioning System (SCS) was used to recirculate the waste and monitor the solids content and particle size of the waste in tank W-9. Once the particle size of a batch of waste was shown to be $<100 \mu \mathrm{m}$ and the total suspended solids (TSS) content was $<5$ to $10 \mathrm{wt} \%$, the batch was allowed to be transferred to the MVSTs through the $\sim 1$ mile long, 2-in.-diam Melton Valley transfer line. The waste acceptance criteria (WAC) for the MVSTs transfer line allow higher TSS contents if data show that settling will not be a problem during the transfer operation.

A variety of waste-retrieval systems and tools were developed and used to retrieve residual wastes from the GAATs. These systems and tooling include the following:

1. Modified Light-Duty Utility Arm (MLDUA)-The MLDUA is an 8 degree of freedom (D.F.) robotic arm with a 200-lb payload and a 15-ft horizontal reach.

Figure 1 is a photograph of the MLDUA, Houdini Vehicle, and Waste Dislodging and Conveyance System (WD\&CS) during cold testing at the ORNL Tanks Technology Cold Test Facility (TTCTF).

2. Houdini Vehicle-Remotely operated vehicle with an integral 6 D.F. manipulator arm with a 240-lb capacity at full extension, on-board camera system, and plow blade. The Houdini Vehicle is a tethered vehicle, which weighs $\sim 1,000 \mathrm{lb}$ and is capable of entering a tank through a 24-in-diam riser.

3. WD\&CS-The WD\&CS includes a water-powered eductor, which is used to vacuum waste from the GAATs; a Confined Sluicing End-Effector (CSEE) for breaking up and retrieving the waste; and the Hose Management Arm (HMA) for support of end effectors and hoses.

a. CSEE-Rotating water-jet cutter and vacuum head for retrieving waste. Water jets supplied with 200-7,000-psi water are used to break up the sludge, which is then vacuumed from the tank.

b. HMA-The discharge conduit for the CSEE is a 4-D.F. pipe arm with a hose distal section. The HMA is designed to support the weight of the conduit arm and sludge as the sludge is transferred out of the tank. The HMA includes an axial-flow, jet-pump operating with 7,000-psi motive water to convey the sludge from the CSEE to the receiving tank (W-9).

4. SCS-The SCS is stationed at the waste consolidation tank (W-9) and is used to mobilize, retrieve, condition, and characterize the waste before its transfer to the MVSTs. The system uses PulsAir ${ }^{\mathrm{TM}}$ Mixers and a Flygt ${ }^{\mathrm{TM}}$ Mixer to mobilize the 
sludge, a submersible pump to transfer the slurry from the tank, a Primary Conditioning System (PCS) unit to filter and sample the slurry, a Solids Monitoring System to determine the solids content and approximate particle-size distribution for the slurry, and other process instrumentation for measuring pressure, temperature, flow rate, etc.

a. PulsAir Mixer-A total of 13 pulse plates $\sim 1 \mathrm{ft}$ in diam are positioned around the bottom of tank W-9 to agitate the solids in W-9 using pressurized air. Figure 2 is a photograph of one of the PulsAir Mixer units at the GAAT site prior to the installation of the unit.

b. Submersible Pump-A 125 -hp electric motor with an integral Discflo ${ }^{\mathrm{TM}}$ lowshear pump is used to transfer slurries from W-9. The mast-mounted pump is capable of generating in excess of 300 psi of discharge pressure. Figure 3 is a drawing of the DiscFlo pump.

c. PCS - The PCS (Fig. 4) includes a parallel roughing filter, three sample ports, high-efficiency particulate air (HEPA) filter air inlet, and containment box.

d. Slurry Monitoring Test Loop (SMTL) - The SMTL (Fig. 5) includes in-line instrumentation for measuring solids content and particle-size distribution and count. The SMTL also includes a sample port, HEPA filter air inlet, and containment box.

5. Flygt Mixers-These mixers have been used in tanks W-5 and W-9 to mobilize the solids for transfer. The mixers include a mast-mounted 15-hp submersible electric motor with a direct drive and an axial flow propeller to agitate the waste in the tank. Figure 6 is a photograph of the mixer before its installation in GAAT W-5.

6. Gunite Scarifying End Effector (GSEE) - The GSEE is used to scarify the contaminated Gunite from the tank walls using high-pressure water at pressures up to 22,000 psi. A high-pressure pump is used to feed clean water to the system.

7. Wall-Coring Tool-The wall-coring tool consists basically of a modified electric drill and collection system, which is used to remove core samples from the walls of the GAATs. The core samples are then analyzed to determine the degree of migration of radioactive components.

8. Wall-Scraping Tool-The wall-scraping tool is used to scrape and collect scale samples from the walls of the GAATs.

9. Pipe-Cutting Tool-The pipe-cutting tool (Fig. 7) consists of a modified electric band saw, which is used to cut piping obstructions inside the GAATs.

10. Pipe-Plugging Tool-The pipe-plugging tool (Fig. 8) is a device, which can be used to seal piping that protrudes inside the GAATs.

11. Linear Scarifying End-effector (LSEE) - The LSEE (Fig. 9) is a 10-ft-long tool, which can be deployed with the Houdini Vehicle system for performing a highpressure wash of a vertical strip of tank wall

The MLDUA and Houdini Vehicle are the primary platforms for placing and operating the various tools and end-effectors used in the remediation of the GAATs.

The last GAAT to be cleaned will be waste-consolidation tank W-9. A large fraction of the wastes transferred to W-9 has settled to the bottom of the tank on top of the original sludge layer present in the tank. These wastes may not be easily resuspended and mixed with supernatant using the PulsAir and Flygt mixers in the time frame allotted for completing waste-removal operations. Therefore, various options for retrieving and transferring wastes from W-9 have been evaluated, and a recommended path has been selected for completion of the GAATs Remediation Project. 
ORNL Photo-6336-2000

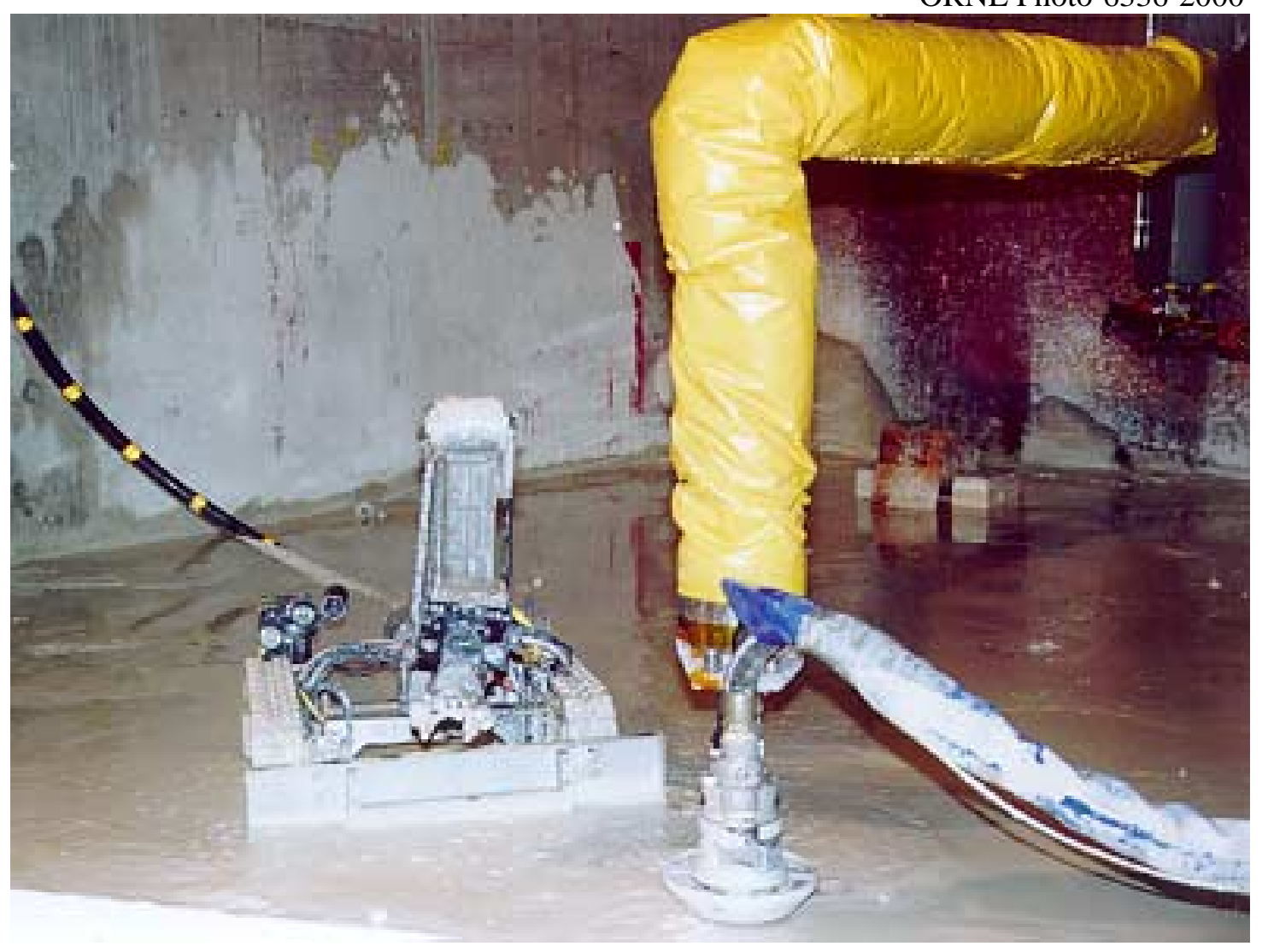

Fig. 1. MLDUA holding the CSEE and operating in conjunction with the Houdini Vehicle at the ORNL TTCTF. 


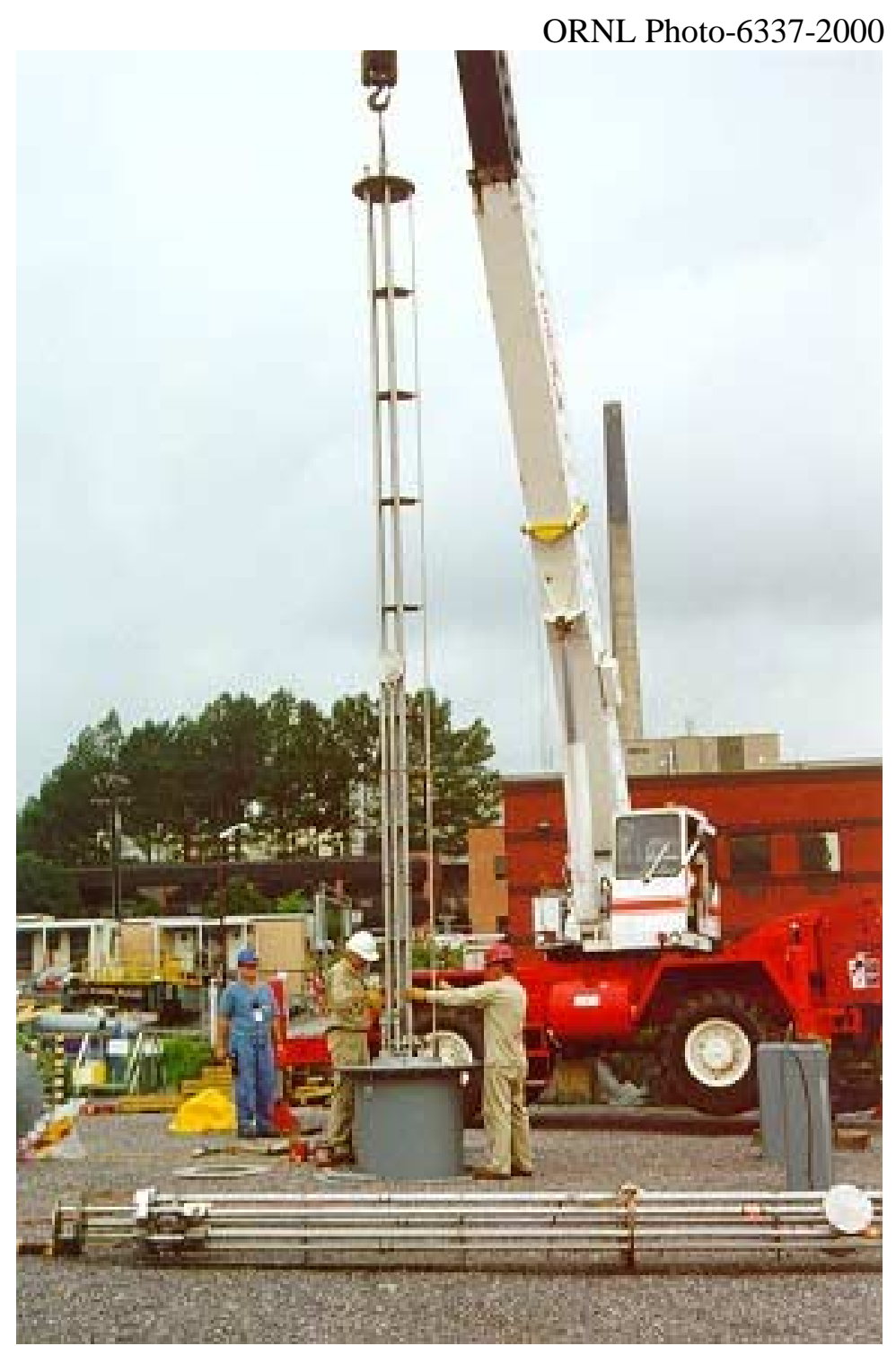

Fig. 2. PulsAir Mixer during installation at GAAT W-9. 
ORNL Dwg-2000-5332

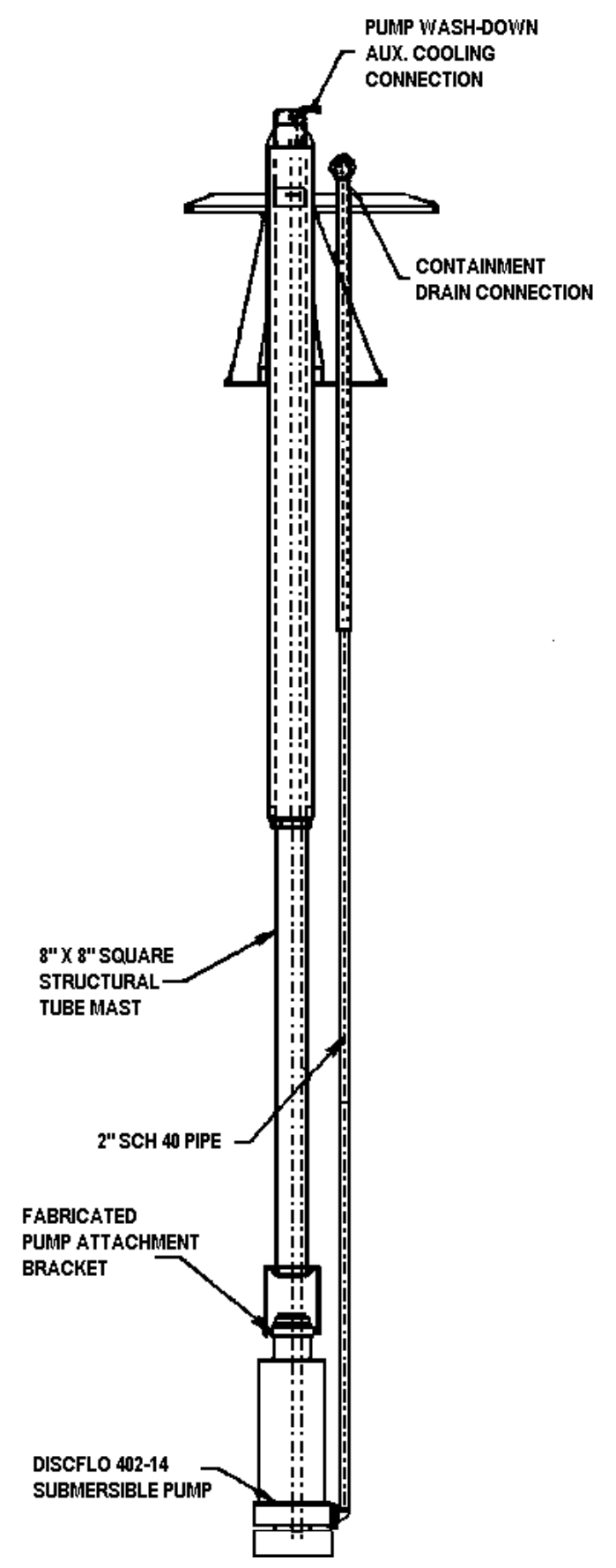

Fig. 3. Sketch of DiscFlo pump. 


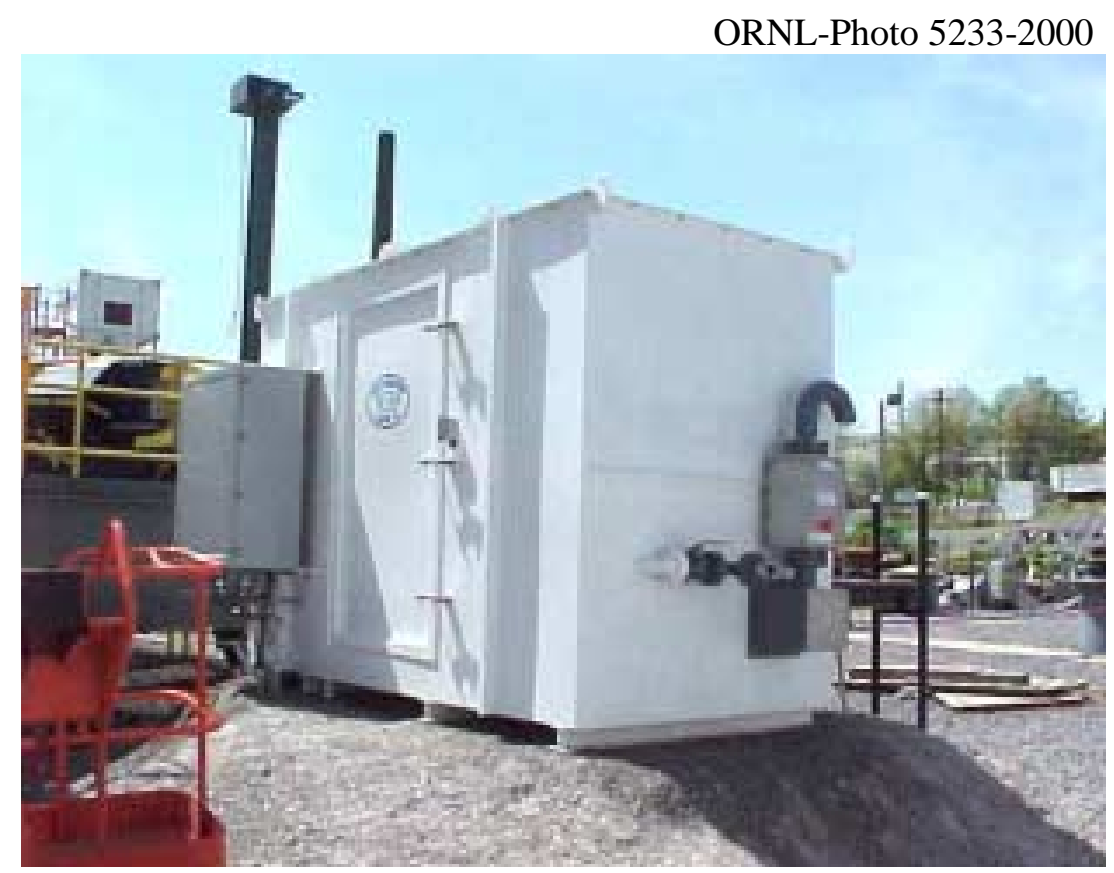

Fig. 4. Primary SCS module.

ORNL Photo-5232-2000

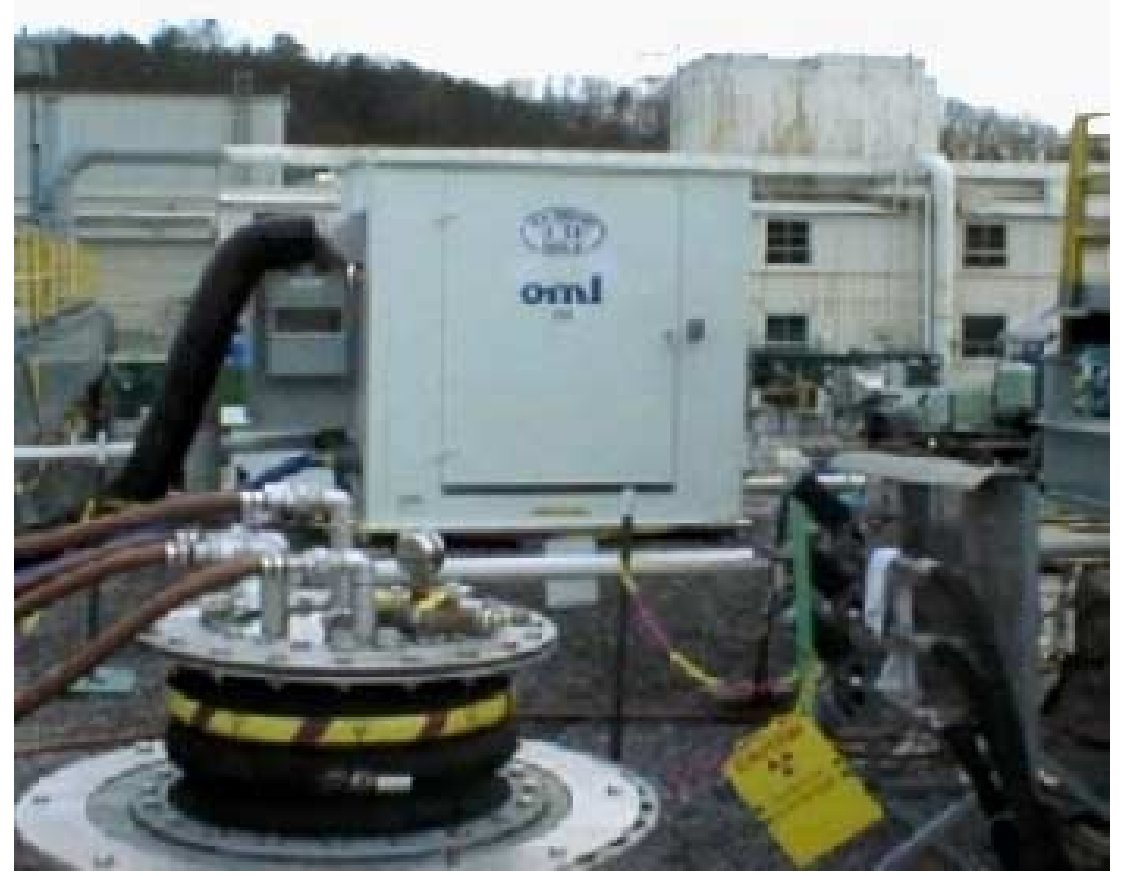

Fig. 5. SMTL module. 
ORNL Photo-6338-2000

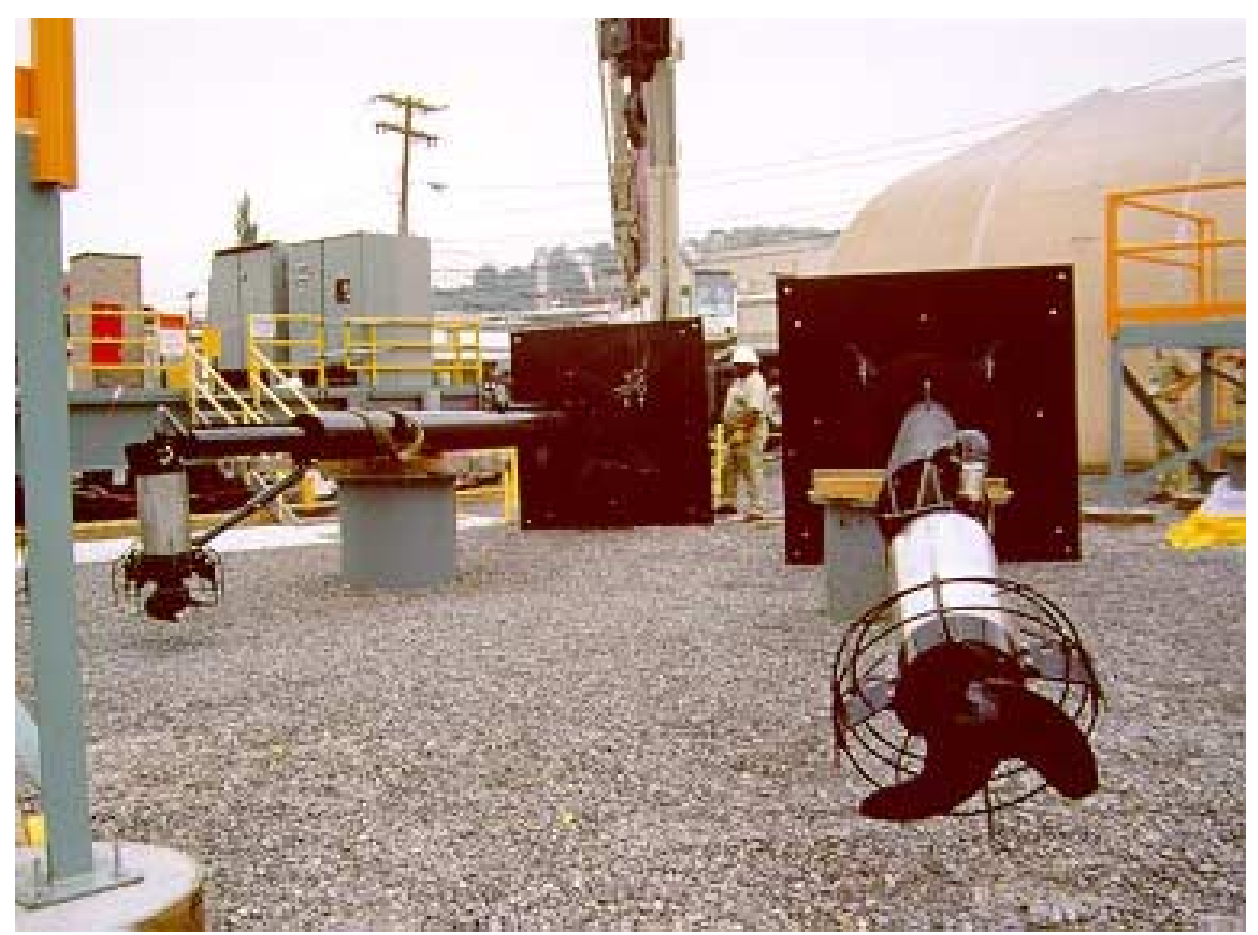

Fig. 6. Flygt mixers before their installation in GAAT W-5.

ORNL Photo-6339-2000

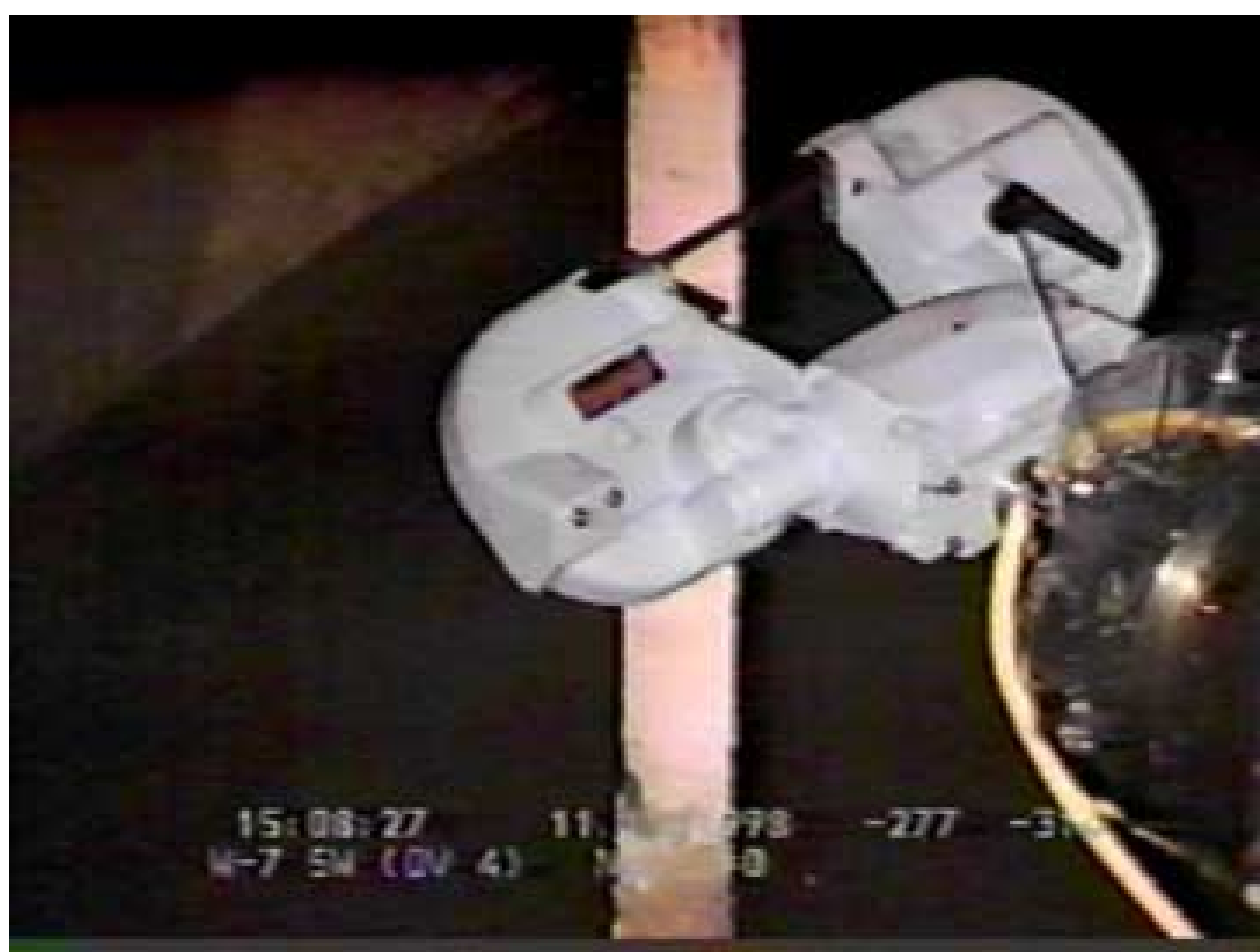

Fig. 7. Pipe-cutting tool in use in GAAT W-7. 

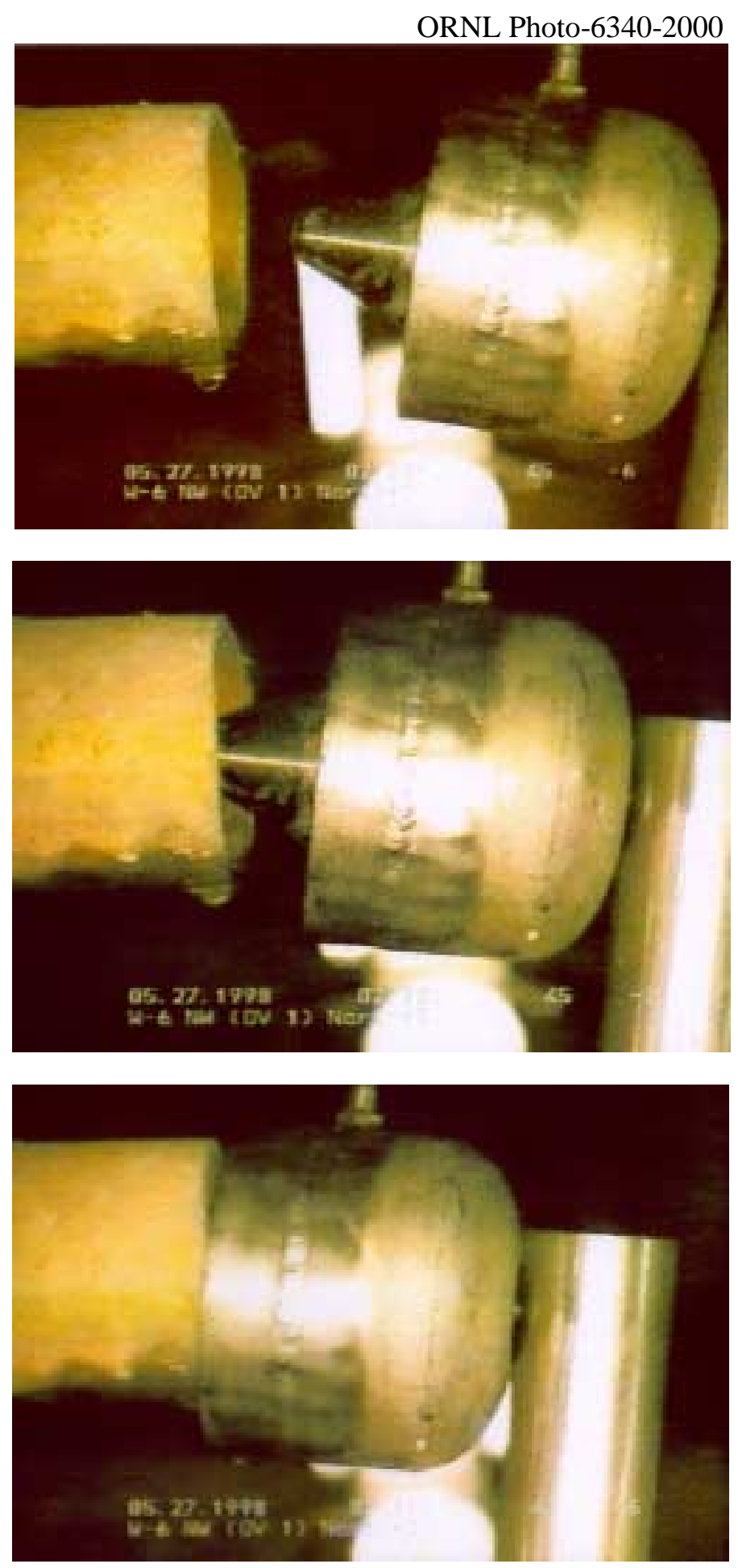

Fig. 8. Sequence of photographs of pipe-plugging tool in use inside GAAT W-6. 
ORNL Photo-6673-2000

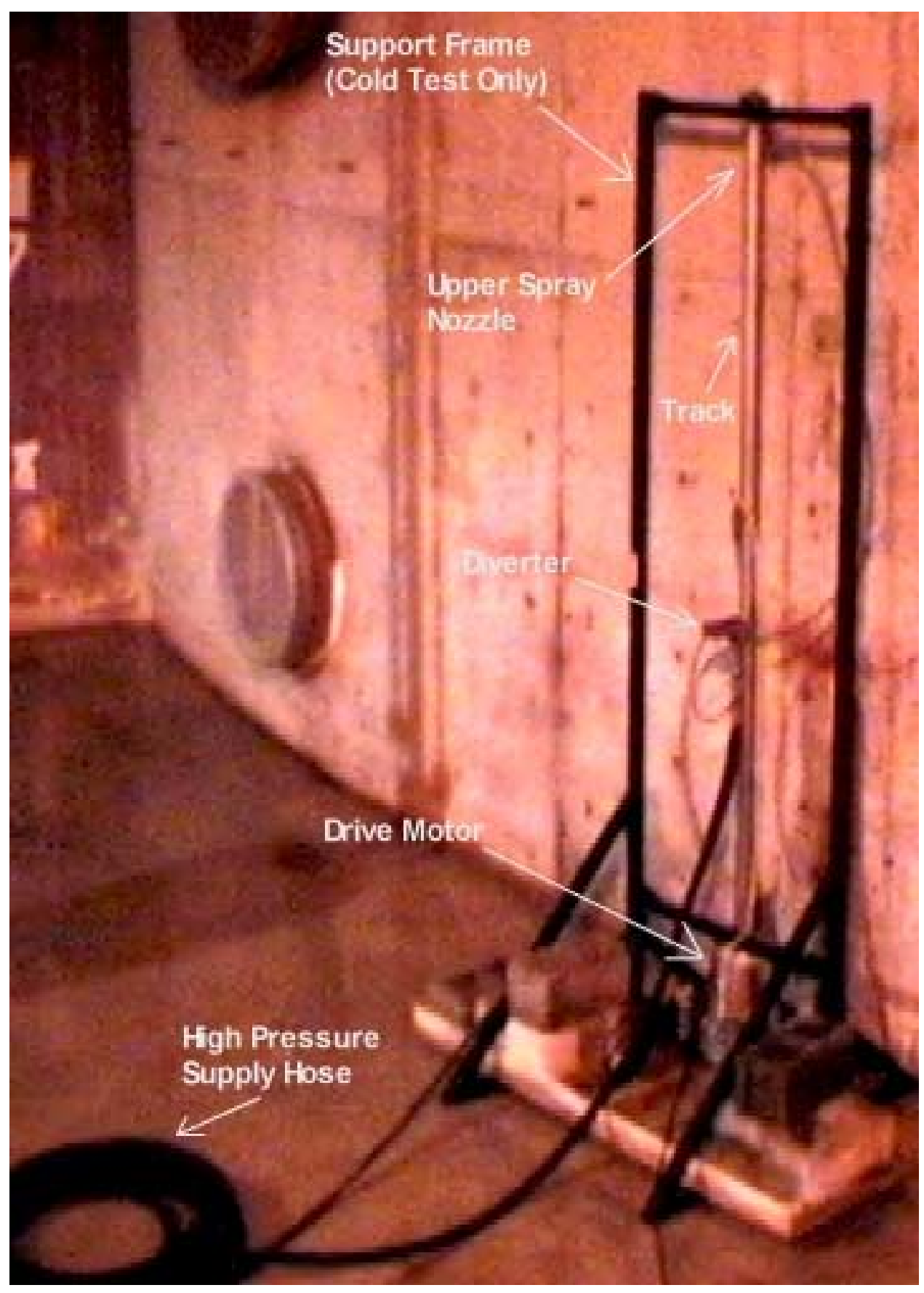

Fig. 9. LSEE at the ORNL TTCTF. 


\section{WASTE-CONSOLIDATION TANK DESCRIPTION AND WASTE- CHARACTERIZATION DATA}

The GAAT Waste Consolidation Tank (W-9) is a 50-ft diam, vertical Gunite tank, which is located in the South Tank Farm (STF) area of ORNL. The tank has 12-ft high walls and a 6-fthigh domed roof and is covered by $\sim 6 \mathrm{ft}$ of soil and crushed limestone. The nominal capacity of the tank is 170,000 gal. Sludge and supernatant from tanks W-3, W-4, W-5, W-6, W-7, W-8, and $\mathrm{W}-10$ have been transferred to W-9 during the tank-waste-retrieval operations. The consolidated waste was then periodically transferred to the MVSTs by the SCS. Before the tank-wasteretrieval and waste-consolidation operations, it was estimated that tank W-9 contained $\sim 2,861$ gal of wet sludge. At the end of the GAAT waste-transfer and consolidation operations in March 2000 , it was estimated that tank W-9 contained $\sim 40,000$ gal of wet sludge and recycled supernate from previous sluicing operations. A variety of samples of the original sludge present in W-9 were taken in 1988, 1994, and $1995 .^{2-5}$ The results from analysis of these samples were combined to show the sludge to have the physical properties and chemical compositions, as given in Table 1.

Additional samples of the accumulated sludge in W-9 were taken near the end of fiscal year (FY) 1999. Wet sludge samples were taken at 3 different depths using 18-in.-long push-tube samplers. The samples represent a vertical profile of the sludge immediately below the north riser of tank $\mathrm{W}-9$. The first sample was collected near the sludge-supernate interface (36 in. from the tank bottom), the second sample was collected near the center of the sludge layer (18 in. from the tank bottom), and the third sample was collected from the tank floor. Various tests and analyses were done to assess the retrievability and pumpability of the sludge. An outline of the analysis plan for the W-9 sludge samples is given in the Appendix 1. Analytical results were obtained for the sample collected from the bottom of the tank and the sample collected $18 \mathrm{in}$. off the bottom of the tank. The sample collected at the 36-in. depth was observed to contain mostly supernate and fine sludge particles. The bottom 3 in. of the lower sample was the most viscous material and was treated as a separate sample. The remaining 15 in. of the lower sample and bottom 4 in. of the middle sample contained mostly previously mixed sludge from the other GAATs. These two sample components were combined and analyzed. The remaining material was primarily supernatant and was not analyzed.

In general, the sludges sampled in FY 1999 were easily dissolved in $4 M$ and $8 M \mathrm{HNO}_{3}$ with very little acid consumption. Due to the limited sample volumes used in the dissolution tests, it is estimated that the error in the amount of acid consumed is $\sim 50 \%$. No measurable change in the number of moles of hydronium ions was determined for the $8 M \mathrm{HNO}_{3}$ tests; this lack of change indicates that minimal acid consumption occurred at this concentration. Photographs of the sludge and supernate samples before and after acid digestion are shown in Fig. 10. Photographs of the sludge and supernatant samples after acid digestion, settling, and centrifugation are shown in Fig. 11. Very little off-gas was observed during the dissolution reactions. The majority of the metals and isotopes were at or near 100\% dissolution, as indicated in Tables 2 and 3. Iron and ${ }^{60} \mathrm{Co}$ were the exceptions in one of the tests. These exceptions may be attributed to a small piece of metal present in the dissolution aliquot.

During the rheological evaluations, both samples held their shape and had a gel-like appearance. When a star shaped probe was inserted into the sample, it was noted that the void created by the probe did not back-fill. Also, shear thickening was noted during the viscosity test on the 
composite sample from the middle of the sludge layer. Maximum shear strengths of 0.035 and $0.127 \mathrm{~Pa}$ were measured for recently suspended/mixed sludge from the middle of the sludge layer and undisturbed sludge from the bottom 3 in. of W-9, respectively. An apparent true viscosity of $\sim 99.8 \mathrm{mPa}$ was measured with the sample stirred at $26^{\circ} \mathrm{C}$. Some shear thickening was also observed for the lower sludge sample. This thickening indicates an increase in the difficulty of mixing with increased agitation.

Analytical data from samples taken from the slurries transferred from tank W-9 to the MVSTs have shown that most of the solids transferred were less than 50 to $100 \mu \mathrm{m}$ in diameter. Selected analytical data from these samples are summarized in Table 4. These data have also been generally corroborated by the solid content data collected from the SCS-SMTL instrumentation. Fig. 12 presents a comparison of the solids content data from the LasenTec ${ }^{\mathrm{TM}}$ instrument in the SMTL and analytical data on the sludge samples from W-9. As shown in Fig. 12, the LasenTec particle count data are offset from the analytical data for particle concentration by an average of $\sim 18,000 \pm 5,330$ particles. The offset is due to differences in the basis and methodology for the particle count. However, the LasenTec data have been used to successfully track the analytical data and provide an indication of when potentially high sludge concentrations can be anticipated. All the sludge sample data collected from the analysis of sludge samples taken during transfers from W-9 through March 30, 2000, are summarized in Appendix 2. The data in Table 4 and Appendix 2 definitely show an increase in the fraction of larger particles over time. However, the fraction of particles $>100 \mu \mathrm{m}$ was never observed to be greater than 2 to $3 \%$. There is also a positive but weak correlation between the fraction of large particles and TSS present. One would expect that mixing in a large volume like GAAT W-9 might tend to classify the solids by density and particle size-with the lower density and smaller particles being held in suspension better and, therefore, pumped off, leaving a higher concentration of more dense and larger particles. If the mixing is complete and is maintained throughout the pump-down of each batch, the effect would be avoided, but that clearly isn't the case here, where there is a definite bed of sludge below the mixed slurry. Table 5 provides a summary of the estimated quantities of sludge and radioactivity transferred from $\mathrm{W}-9$ by the SCS. 
Table 1. Characteristics of the original wet sludge present in GAAT W-9 prior to consolidation

\begin{tabular}{|c|c|c|c|c|c|}
\hline Characteristic & $\begin{array}{c}\text { W-9 } \\
\text { Average }\end{array}$ & Characteristic & $\begin{array}{c}\text { W-9 } \\
\text { Average }\end{array}$ & Characteristic & $\begin{array}{c}\text { W-9 } \\
\text { Average }\end{array}$ \\
\hline General characteristics & & Alkali metals (mg/kg) & & Anions (mg/kg) & \\
\hline Wet sludge volume (L) & 10,829 & ${ }^{137} \mathrm{Cs}(\mathrm{Bq} / \mathrm{g})$ & 350,000 & Bromide & 7 \\
\hline Water content $(\%)$ & 85.37 & ${ }^{137} \mathrm{Cs}$ & 0.1087 & Chloride & 138 \\
\hline Density $(\mathrm{g} / \mathrm{mL})$ & 1.21 & Sodium & 6,340 & Fluoride & 155 \\
\hline Wet sludge mass $(\mathrm{kg})$ & 13,103 & Potassium & 2,987 & Nitrate & 436 \\
\hline $\mathrm{pH}$ & 10.00 & $\begin{array}{l}\text { Subtotal }(\mathrm{mg} / \mathrm{kg}): \\
\text { Subtotal }(\mathrm{g} / \mathrm{g} \text {-wet }):\end{array}$ & $\begin{array}{r}9,327 \\
0.0093\end{array}$ & $\begin{array}{l}\text { Phosphate } \\
\text { Sulphate }\end{array}$ & $\begin{array}{r}2,960 \\
536 \\
\end{array}$ \\
\hline Carbon content (mg/L) & & Subtotal (g/g-dry): & 0.0638 & Subtotal (mg/kg): & 4,232 \\
\hline $\mathrm{TOC}^{a}$ & 2,650 & & & Subtotal (g/g-wet): & 0.0042 \\
\hline $\mathrm{TIC}^{b}$ & 1,890 & Alkaline earths (mg/kg) & & Subtotal (g/g-dry): & 0.0289 \\
\hline Subtotal (mg/L): & 4,540 & Beryllium & 6.57 & & \\
\hline Subtotal (g/g-wet): & 0.0038 & Calcium & 6,150 & Mass balance (g/g-wet): & 0.9163 \\
\hline Subtotal (g/g-dry): & 0.0256 & $\begin{array}{l}\text { Magnesium } \\
\text { Strontium }\end{array}$ & $\begin{array}{r}767.0 \\
39.4 \\
\end{array}$ & Mass balance (g/g-dry): & 0.4278 \\
\hline $\begin{array}{l}\text { RCRA }^{c} \text { Constituents } \\
(\mathbf{m g} / \mathbf{k g})\end{array}$ & & Subtotal $(\mathrm{mg} / \mathrm{kg})$ : & 6,963 & Radioactivity ( $\mathrm{\eta Ci} / \mathrm{g}$ ) & \\
\hline Arsenic $($ limit $=5 \mathrm{ppm})$ & & Subtotal (g/g-wet): & 0.0070 & Gross alpha & 2,036 \\
\hline Barium (100) & 100.97 & Subtotal (g/g-dry): & 0.0476 & Gross beta & 132,432 \\
\hline Cadmium (1) & 3.1 & & & ${ }^{60} \mathrm{Co}$ & 191.89 \\
\hline Chromium (5) & 120 & $\begin{array}{l}\text { Other constituents } \\
(\mathrm{mg} / \mathrm{kg})\end{array}$ & & ${ }^{90} \mathrm{Sr} /{ }^{90} \mathrm{Y}$ & 45,946 \\
\hline Lead (5) & 496 & Aluminum & 8,847 & ${ }^{137} \mathrm{Cs}$ & 9,459 \\
\hline Mercury (0.2) & 66.7 & Boron & 6.66 & ${ }^{152} \mathrm{Eu}$ & 100.00 \\
\hline Nickel (5) & 72.7 & Cobalt & 2.57 & ${ }^{154} \mathrm{Eu}$ & 135.14 \\
\hline Selenium (1) & & Copper & 48.4 & ${ }^{155} \mathrm{Eu}$ & \\
\hline Silver (5) & & Iron & 3,207 & ${ }^{244} \mathrm{Cm}$ & $1,300.19$ \\
\hline Thallium (0.9) & & Manganese & 146.3 & ${ }^{238} \mathrm{Pu},{ }^{241} \mathrm{Am}$ & 490.73 \\
\hline Subtotal $(\mathrm{mg} / \mathrm{kg})$ : & 859 & Silicon & & ${ }^{239} \mathrm{Pu},{ }^{240} \mathrm{Pu}$ & 221.84 \\
\hline Subtotal (g/g-wet): & 0.0009 & Thorium & 5,970 & Total $\mathrm{Pu}$ & 675.68 \\
\hline \multirow[t]{6}{*}{ Subtotal (g/g-dry): } & 0.0059 & Uranium & 19,167 & \multirow{5}{*}{\begin{tabular}{|}
$\mathrm{TRU}^{d}(\mathrm{Pu} / \mathrm{Am}$ based $)$ \\
$\mathbf{P C B}^{e}(\mathbf{m g} / \mathbf{k g})$ \\
AROCLOR -1248 \\
AROCLOR - 1254 \\
AROCLOR - 1260
\end{tabular}} & 713 \\
\hline & & $\begin{array}{l}\text { Vanadium } \\
\text { Zinc }\end{array}$ & 56.0 & & \\
\hline & & \multirow{4}{*}{$\begin{array}{l}\text { Subtotal (mg/kg): } \\
\text { Subtotal (g/g-wet): } \\
\text { Subtotal (g/g-dry): }\end{array}$} & \multirow{4}{*}{$\begin{array}{r}37,451 \\
0.0375 \\
0.2560\end{array}$} & & \\
\hline & & & & & 0.1 \\
\hline & & & & & \\
\hline & & & & Total AROCLORs & 0.1 \\
\hline
\end{tabular}

${ }^{a}$ Total organic carbon

${ }^{b}$ Total inorganic carbon

${ }^{c}$ Resource Conservation and Recovery Act

${ }^{d}$ Transuranic

${ }^{e}$ Polychlorinated biphenyl 
Table 2. Dissolution data for W-9 consolidated sludge sample 990825-014

\begin{tabular}{|c|c|c|c|c|c|c|c|}
\hline \multirow{4}{*}{\begin{tabular}{|c|} 
Analysis \\
\\
Miscellaneous \\
pH
\end{tabular}} & \multirow{3}{*}{\begin{tabular}{c|} 
GAAT- \\
W9-049 \\
990825-014
\end{tabular}} & \multicolumn{3}{|c|}{$4 M$ Dissolution $^{a, b}$} & \multicolumn{3}{|c|}{$8 M$ Dissolution $^{a, b}$} \\
\hline & & Liquid & Solids & $\begin{array}{c}\text { Percentage } \\
\text { dissolved }\end{array}$ & Liquid & Solids & $\begin{array}{l}\text { Percentage } \\
\text { dissolved }\end{array}$ \\
\hline & & \multicolumn{3}{|c|}{ All results corrected back to original aliquot } & \multicolumn{3}{|c|}{ All results corrected back to original aliquot } \\
\hline & & & & & & & \\
\hline Percentage solids & $2.02 \mathrm{E}+01$ & & & & & & \\
\hline TSS (\%) & $1.92 \mathrm{E}+01$ & & $3.6 \mathrm{E}+00$ & $8.1 \mathrm{E}+01$ & & $1.8 \mathrm{E}+00$ & $9.1 \mathrm{E}+01$ \\
\hline Density $(g / m L)$ & $1.167 \mathrm{E}+00$ & & & & & & \\
\hline $\begin{array}{l}\text { Acid } \\
\text { consumption }\end{array}$ & & & & $1.8 \mathrm{E}-03 \mathrm{Mol}$ & & & No change \\
\hline \multicolumn{8}{|l|}{ RCRA metals (mg/L) } \\
\hline $\mathbf{B a}$ & $5.57 \mathrm{E}+01$ & $3.28 \mathrm{E}+01$ & ND & $1.0 \mathrm{E}+02$ & $3.43 \mathrm{E}+01$ & ND & $1.0 \mathrm{E}+02$ \\
\hline Cd & $2.96 \mathrm{E}+01$ & $1.95 \mathrm{E}+01$ & ND & $1.0 \mathrm{E}+02$ & $2.13 \mathrm{E}+01$ & ND & $1.0 \mathrm{E}+02$ \\
\hline $\mathbf{C r}$ & $2.86 \mathrm{E}+02$ & $1.79 \mathrm{E}+02$ & ND & $1.0 \mathrm{E}+02$ & $1.91 \mathrm{E}+02$ & $8.23 \mathrm{E}+00$ & $9.6 \mathrm{E}+01$ \\
\hline $\mathrm{Ni}$ & $4.69 \mathrm{E}+01$ & $4.01 \mathrm{E}+01$ & ND & $1.0 \mathrm{E}+02$ & $4.35 \mathrm{E}+01$ & ND & $1.0 \mathrm{E}+02$ \\
\hline $\mathbf{P b}$ & $8.78 \mathrm{E}+01$ & $1.79 \mathrm{E}+02$ & ND & $1.0 \mathrm{E}+02$ & $2.03 \mathrm{E}+02$ & ND & $1.0 \mathrm{E}+02$ \\
\hline \multicolumn{8}{|l|}{ Process metals $(\mathrm{mg} / \mathrm{L})$} \\
\hline $\mathbf{A l}$ & $8.96 \mathrm{E}+03$ & $5.32 \mathrm{E}+03$ & $5.57 \mathrm{E}+00$ & $1.0 \mathrm{E}+02$ & $6.00 \mathrm{E}+03$ & $7.23 E+02$ & $8.9 \mathrm{E}+01$ \\
\hline B & $1.26 \mathrm{E}+01$ & $1.76 \mathrm{E}+00$ & ND & $1.0 \mathrm{E}+02$ & $1.70 \mathrm{E}+00$ & ND & $1.0 \mathrm{E}+02$ \\
\hline Be & $3.61 \mathrm{E}+00$ & $4.69 \mathrm{E}+00$ & ND & $1.0 \mathrm{E}+02$ & $5.04 \mathrm{E}+00$ & ND & $1.0 \mathrm{E}+02$ \\
\hline $\mathbf{C a}$ & $2.93 \mathrm{E}+03$ & $2.47 \mathrm{E}+03$ & $2.57 \mathrm{E}+01$ & $9.9 \mathrm{E}+01$ & $2.54 \mathrm{E}+03$ & $2.15 \mathrm{E}+01$ & $9.9 \mathrm{E}+01$ \\
\hline Co & $3.89 \mathrm{E}+01$ & $1.66 \mathrm{E}+01$ & ND & $1.0 \mathrm{E}+02$ & $1.70 \mathrm{E}+01$ & ND & $1.0 \mathrm{E}+02$ \\
\hline $\mathbf{C u}$ & $1.11 \mathrm{E}+02$ & $2.25 \mathrm{E}+01$ & $7.68 \mathrm{E}-01$ & $9.7 \mathrm{E}+01$ & $2.44 \mathrm{E}+01$ & $1.57 \mathrm{E}+00$ & $9.4 \mathrm{E}+01$ \\
\hline $\mathbf{F e}$ & $1.81 \mathrm{E}+03$ & $1.07 \mathrm{E}+03$ & $5.47 \mathrm{E}+00$ & $9.9 \mathrm{E}+01$ & $1.08 \mathrm{E}+03$ & $3.88 \mathrm{E}+02$ & $7.4 \mathrm{E}+01$ \\
\hline $\mathbf{K}$ & $2.52 \mathrm{E}+03$ & $2.21 \mathrm{E}+03$ & ND & $1.0 \mathrm{E}+02$ & $2.28 \mathrm{E}+03$ & $2.81 \mathrm{E}+01$ & $9.9 \mathrm{E}+01$ \\
\hline Mg & $1.44 \mathrm{E}+03$ & $4.92 \mathrm{E}+02$ & ND & $1.0 \mathrm{E}+02$ & $5.21 \mathrm{E}+02$ & $4.24 \mathrm{E}+01$ & $9.2 \mathrm{E}+01$ \\
\hline Mn & $1.88 \mathrm{E}+02$ & $1.42 \mathrm{E}+02$ & ND & $1.0 \mathrm{E}+02$ & $1.49 \mathrm{E}+02$ & $1.86 \mathrm{E}+00$ & $9.9 \mathrm{E}+01$ \\
\hline Mo & $5.18 \mathrm{E}+02$ & $3.42 \mathrm{E}+01$ & ND & $1.0 \mathrm{E}+02$ & $3.39 \mathrm{E}+01$ & ND & $1.0 \mathrm{E}+02$ \\
\hline $\mathbf{N a}$ & $1.53 \mathrm{E}+04$ & $1.02 \mathrm{E}+04$ & ND & $1.0 \mathrm{E}+02$ & $1.11 \mathrm{E}+04$ & $1.10 \mathrm{E}+01$ & $1.0 \mathrm{E}+02$ \\
\hline $\mathbf{S r}$ & $2.70 \mathrm{E}+01$ & $2.02 \mathrm{E}+01$ & ND & $1.0 \mathrm{E}+02$ & $2.13 \mathrm{E}+01$ & ND & $1.0 \mathrm{E}+02$ \\
\hline Th & $2.09 \mathrm{E}+03$ & $1.55 \mathrm{E}+03$ & ND & $1.0 \mathrm{E}+02$ & $1.75 \mathrm{E}+03$ & $4.40 \mathrm{E}+01$ & $9.8 \mathrm{E}+01$ \\
\hline $\mathbf{U}$ & $1.05 \mathrm{E}+05$ & $8.11 \mathrm{E}+04$ & ND & $1.0 \mathrm{E}+02$ & $8.57 \mathrm{E}+04$ & $3.49 \mathrm{E}+01$ & $1.0 \mathrm{E}+02$ \\
\hline $\mathbf{V}$ & $9.74 \mathrm{E}+00$ & ND & ND & ND & ND & ND & ND \\
\hline $\mathbf{Z n}$ & ND & ND & ND & ND & ND & ND & ND \\
\hline \multicolumn{8}{|c|}{ General radiochemical $(\mathrm{Bq} / \mathrm{g})$} \\
\hline Total activity & $2.8 \mathrm{E}+06$ & $1.8 \mathrm{E}+06$ & $3.0 \mathrm{E}+03$ & $1.0 \mathrm{E}+02$ & $1.9 \mathrm{E}+06$ & $3.8 \mathrm{E}+04$ & $9.8 \mathrm{E}+01$ \\
\hline Gross alpha & $3.0 \mathrm{E}+04$ & $2.5 \mathrm{E}+04$ & $6.8 \mathrm{E}+01$ & $1.0 \mathrm{E}+02$ & $2.4 \mathrm{E}+04$ & $3.7 \mathrm{E}+02$ & $9.8 \mathrm{E}+01$ \\
\hline \multicolumn{8}{|c|}{ Beta/gamma emitters (Bq/g) } \\
\hline${ }^{60} \mathrm{Co}$ & $1.6 \mathrm{E}+03$ & $1.2 \mathrm{E}+03$ & ND & $1.0 \mathrm{E}+02$ & $1.2 \mathrm{E}+03$ & $4.6 \mathrm{E}+01$ & $9.6 \mathrm{E}+01$ \\
\hline${ }^{137} \mathrm{Cs}$ & $2.1 \mathrm{E}+05$ & $1.4 \mathrm{E}+05$ & $1.9 \mathrm{E}+03$ & $9.9 \mathrm{E}+01$ & $1.6 \mathrm{E}+05$ & $1.9 \mathrm{E}+04$ & $8.9 \mathrm{E}+01$ \\
\hline${ }^{152} \mathbf{E u}$ & $1.2 \mathrm{E}+03$ & $1.2 \mathrm{E}+03$ & ND & $1.0 \mathrm{E}+02$ & $1.3 \mathrm{E}+03$ & ND & $1.0 \mathrm{E}+02$ \\
\hline${ }^{154} \mathbf{E u}$ & $1.5 \mathrm{E}+03$ & $1.0 \mathrm{E}+03$ & ND & $1.0 \mathrm{E}+02$ & $1.5 \mathrm{E}+03$ & ND & $1.0 \mathrm{E}+02$ \\
\hline \multicolumn{8}{|c|}{ Alpha emitters (Bq/g) } \\
\hline$\frac{1}{238} \mathbf{U}$ & $1.0 \mathrm{E}+03$ & $9.3 \mathrm{E}+02$ & ND & $1.0 \mathrm{E}+02$ & $7.9 \mathrm{E}+02$ & $1.9 \mathrm{E}+00$ & $1.0 \mathrm{E}+02$ \\
\hline${ }^{233} \mathbf{U} /{ }^{234} \mathrm{U}$ & $1.5 \mathrm{E}+03$ & $1.2 \mathrm{E}+03$ & $1.6 \mathrm{E}+00$ & $1.0 \mathrm{E}+02$ & $1.4 \mathrm{E}+03$ & $9.6 \mathrm{E}+00$ & $9.9 \mathrm{E}+01$ \\
\hline${ }^{239} \mathrm{Pu} /{ }^{240} \mathrm{Pu}$ & $5.3 \mathrm{E}+03$ & $4.5 \mathrm{E}+03$ & $5.8 \mathrm{E}+01$ & $9.9 \mathrm{E}+01$ & $4.1 \mathrm{E}+03$ & $1.3 \mathrm{E}+02$ & $9.7 \mathrm{E}+01$ \\
\hline${ }^{238} \mathrm{Pu}-/{ }^{241} \mathrm{Am}$ & $7.2 \mathrm{E}+03$ & $6.0 \mathrm{E}+03$ & $7.8 \mathrm{E}+00$ & $1.0 \mathrm{E}+02$ & $5.2 \mathrm{E}+03$ & $1.3 \mathrm{E}+02$ & $9.8 \mathrm{E}+01$ \\
\hline${ }^{244} \mathrm{Cm}$ & $1.5 \mathrm{E}+04$ & $1.2 \mathrm{E}+04$ & $8.2 \mathrm{E}-01$ & $1.0 \mathrm{E}+02$ & $1.3 \mathrm{E}+04$ & $9.5 \mathrm{E}+01$ & $9.9 \mathrm{E}+01$ \\
\hline
\end{tabular}

${ }^{a}$ A 5:1 ratio acid to sludge was used for the tests. Mixtures were shaken, centrifuged and the phases separated for analysis. Actual acid concentrations were $3.97 \mathrm{M}$ and $8.03 \mathrm{M}$ respectively.

${ }^{b} \mathrm{ND}$ indicates that the analyte was below the detectable limit of the instrument in the dilution analyzed. 
Table 3. Dissolution data for W-9 consolidated sludge solids sample 990825-015

\begin{tabular}{|c|c|c|c|c|c|c|c|}
\hline \multirow{4}{*}{\begin{tabular}{|c} 
Analysis \\
\\
Miscellaneous \\
pH
\end{tabular}} & \multirow{3}{*}{$\begin{array}{l}\text { W9-049Solid } \\
\text { 990825-015 }\end{array}$} & \multicolumn{3}{|c|}{$4 M$ Dissolution ${ }^{a, b}$} & \multicolumn{3}{|c|}{$8 M$ Dissolution $^{a, b}$} \\
\hline & & Liquid & Solids & $\begin{array}{c}\text { Percentage } \\
\text { dissolved }\end{array}$ & Liquid & Solids & $\begin{array}{c}\text { Percentage } \\
\text { dissolved }\end{array}$ \\
\hline & & \multicolumn{3}{|c|}{ All results corrected back to original aliquot } & \multicolumn{3}{|c|}{ All results corrected back to original aliquot } \\
\hline & & & & & & & \\
\hline Percentage solids & $4.83 \mathrm{E}+01$ & & & & & & \\
\hline TSS (\%) & $1.0 \mathrm{E}+02$ & & 0.0 & $1.0 \mathrm{E}+02$ & & 0.0 & $1.0 \mathrm{E}+02$ \\
\hline Density (g/mL) & $1.587 \mathrm{E}+00$ & & & & & & \\
\hline $\begin{array}{l}\text { Acid } \\
\text { consumption }\end{array}$ & & & & $1.0 \mathrm{E}-03 \mathrm{~mol}$ & & & No change \\
\hline \multicolumn{8}{|l|}{ RCRA metals (mg/L) } \\
\hline $\mathbf{B a}$ & $3.40 \mathrm{E}+01$ & $1.24 \mathrm{E}+01$ & ND & $1.0 \mathrm{E}+02$ & $1.40 \mathrm{E}+01$ & ND & $1.0 \mathrm{E}+02$ \\
\hline Cd & $4.06 \mathrm{E}+01$ & $3.38 \mathrm{E}+01$ & ND & $1.0 \mathrm{E}+02$ & $3.55 \mathrm{E}+01$ & ND & $1.0 \mathrm{E}+02$ \\
\hline $\mathbf{C r}$ & $2.11 \mathrm{E}+02$ & $5.75 \mathrm{E}+01$ & $1.35 \mathrm{E}+01$ & $8.1 \mathrm{E}+01$ & $6.65 \mathrm{E}+01$ & ND & $1.0 \mathrm{E}+02$ \\
\hline $\mathbf{N i}$ & $7.06 \mathrm{E}+01$ & $5.84 \mathrm{E}+01$ & ND & $1.0 \mathrm{E}+02$ & $6.03 \mathrm{E}+01$ & ND & $1.0 \mathrm{E}+02$ \\
\hline $\mathbf{P b}$ & ND & $9.19 \mathrm{E}+01$ & ND & $1.0 \mathrm{E}+02$ & $1.08 \mathrm{E}+02$ & ND & $1.0 \mathrm{E}+02$ \\
\hline \multicolumn{8}{|c|}{ Process metals (mg/L) } \\
\hline Al & $2.94 \mathrm{E}+03$ & $8.17 \mathrm{E}+02$ & $1.57 \mathrm{E}+03$ & $3.4 \mathrm{E}+01$ & $1.97 \mathrm{E}+00$ & $1.01 \mathrm{E}+01$ & $1.6 \mathrm{E}+01$ \\
\hline B & $4.22 \mathrm{E}+01$ & $5.74 \mathrm{E}+00$ & ND & $1.0 \mathrm{E}+02$ & $5.01 \mathrm{E}+00$ & ND & $1.0 \mathrm{E}+02$ \\
\hline $\mathbf{B e}$ & $8.03 \mathrm{E}+00$ & $9.51 \mathrm{E}+00$ & ND & $1.0 \mathrm{E}+02$ & $1.02 \mathrm{E}+01$ & ND & $1.0 \mathrm{E}+02$ \\
\hline $\mathbf{C a}$ & $8.16 \mathrm{E}+02$ & $5.60 \mathrm{E}+02$ & $8.50 \mathrm{E}+00$ & $9.9 \mathrm{E}+01$ & $7.16 \mathrm{E}+02$ & $1.99 \mathrm{E}+01$ & $9.7 \mathrm{E}+01$ \\
\hline Co & $9.81 \mathrm{E}+01$ & $4.55 \mathrm{E}+01$ & ND & $1.0 \mathrm{E}+02$ & $4.82 \mathrm{E}+01$ & ND & $1.0 \mathrm{E}+02$ \\
\hline $\mathrm{Cu}$ & $3.04 \mathrm{E}+02$ & $7.73 \mathrm{E}+01$ & $3.09 \mathrm{E}+00$ & $9.6 \mathrm{E}+01$ & $4.97 \mathrm{E}+01$ & ND & $1.0 \mathrm{E}+02$ \\
\hline $\mathbf{F e}$ & $2.53 \mathrm{E}+02$ & $1.05 \mathrm{E}+02$ & $6.74 \mathrm{E}+02$ & $1.3 \mathrm{E}+01$ & $1.12 \mathrm{E}+02$ & $5.79 \mathrm{E}+00$ & $9.5 \mathrm{E}+01$ \\
\hline $\mathbf{K}$ & $1.67 \mathrm{E}+04$ & $1.81 \mathrm{E}+04$ & $5.54 \mathrm{E}+01$ & $1.0 \mathrm{E}+02$ & $1.85 \mathrm{E}+04$ & ND & $1.0 \mathrm{E}+02$ \\
\hline Mg & $2.64 \mathrm{E}+03$ & $4.83 \mathrm{E}+02$ & $7.58 \mathrm{E}+01$ & $8.6 \mathrm{E}+01$ & $4.83 \mathrm{E}+02$ & ND & $1.0 \mathrm{E}+02$ \\
\hline Mn & $5.75 \mathrm{E}+01$ & $1.19 \mathrm{E}+01$ & $4.15 \mathrm{E}+00$ & $7.4 \mathrm{E}+01$ & $2.12 \mathrm{E}+01$ & ND & $1.0 \mathrm{E}+02$ \\
\hline Mo & $1.24 \mathrm{E}+03$ & $4.77 \mathrm{E}+01$ & ND & $1.0 \mathrm{E}+02$ & $7.36 \mathrm{E}+01$ & ND & $1.0 \mathrm{E}+02$ \\
\hline $\mathbf{N a}$ & $2.91 \mathrm{E}+04$ & $2.37 \mathrm{E}+04$ & $1.34 \mathrm{E}+01$ & $1.0 \mathrm{E}+02$ & $2.44 \mathrm{E}+04$ & ND & $1.0 \mathrm{E}+02$ \\
\hline $\mathrm{Sr}$ & $1.72 \mathrm{E}+01$ & $1.75 \mathrm{E}+01$ & $1.93 \mathrm{E}-01$ & $9.9 \mathrm{E}+01$ & $1.82 \mathrm{E}+01$ & ND & $1.0 \mathrm{E}+02$ \\
\hline Th & $2.84 \mathrm{E}+02$ & ND & $6.59 \mathrm{E}+01$ & 0.0 & ND & ND & $1.0 \mathrm{E}+02$ \\
\hline $\mathbf{U}$ & $2.69 \mathrm{E}+05$ & $2.63 \mathrm{E}+05$ & $4.09 \mathrm{E}+01$ & $1.0 \mathrm{E}+02$ & $2.68 \mathrm{E}+05$ & ND & $1.0 \mathrm{E}+02$ \\
\hline V & $2.63 \mathrm{E}+01$ & $5.18 \mathrm{E}+00$ & ND & $1.0 \mathrm{E}+02$ & $1.69 \mathrm{E}+00$ & ND & $1.0 \mathrm{E}+02$ \\
\hline Zn & ND & ND & ND & ND & $3.06 \mathrm{E}+01$ & ND & $1.0 \mathrm{E}+02$ \\
\hline \multicolumn{8}{|c|}{ General radiochemical $(\mathrm{Bq} / \mathrm{g})$} \\
\hline Total activity & $3.1 \mathrm{E}+06$ & $2.8 \mathrm{E}+06$ & $6.9 \mathrm{E}+04$ & $9.8 \mathrm{E}+01$ & $2.8 \mathrm{E}+06$ & $4.0 \mathrm{E}+03$ & $1.0 \mathrm{E}+02$ \\
\hline Gross alpha & $1.8 \mathrm{E}+04$ & $1.8 \mathrm{E}+04$ & $6.0 \mathrm{E}+02$ & $9.7 \mathrm{E}+01$ & $2.3 \mathrm{E}+04$ & $4.4 \mathrm{E}+01$ & $1.0 \mathrm{E}+02$ \\
\hline \multicolumn{8}{|c|}{ Beta/gamma emitters $(\mathrm{Bq} / \mathrm{g})$} \\
\hline${ }^{60} \mathrm{Co}$ & ND & $9.3 \mathrm{E}+01$ & $5.2 \mathrm{E}+01$ & $6.4 \mathrm{E}+01$ & $1.3 \mathrm{E}+2$ & ND & $1.0 \mathrm{E}+02$ \\
\hline${ }^{137} \mathrm{Cs}$ & $1.8 \mathrm{E}+06$ & $1.7 \mathrm{E}+06$ & $3.3 \mathrm{E}+04$ & $9.8 \mathrm{E}+01$ & $1.7 \mathrm{E}+6$ & $2.8 \mathrm{E}+03$ & $1.0 \mathrm{E}+02$ \\
\hline \multicolumn{8}{|l|}{ Alpha emitters (Bq/g) } \\
\hline${ }^{238} \mathbf{U}$ & $2.5 \mathrm{E}+03$ & $2.7 \mathrm{E}+03$ & ND & $1.0 \mathrm{E}+02$ & $2.3 \mathrm{E}+03$ & ND & $1.0 \mathrm{E}+02$ \\
\hline${ }^{233} \mathbf{U} /{ }^{234} \mathbf{U}$ & $3.0 \mathrm{E}+03$ & $3.1 \mathrm{E}+03$ & $1.3 \mathrm{E}+01$ & $1.0 \mathrm{E}+02$ & $3.4 \mathrm{E}+03$ & ND & $1.0 \mathrm{E}+02$ \\
\hline${ }^{239} \mathrm{Pu} /{ }^{240} \mathrm{Pu}$ & $9.3 \mathrm{E}+03$ & $9.4 \mathrm{E}+03$ & $2.3 \mathrm{E}+02$ & $9.8 \mathrm{E}+01$ & $1.4 \mathrm{E}+04$ & $3.9 \mathrm{E}+01$ & $1.0 \mathrm{E}+02$ \\
\hline${ }^{238} \mathbf{P u} /{ }^{241} \mathrm{Am}$ & $2.3 \mathrm{E}+03$ & $1.9 \mathrm{E}+03$ & $2.4 \mathrm{E}+02$ & $8.9 \mathrm{E}+01$ & $2.2 \mathrm{E}+03$ & $3.7 \mathrm{E}+00$ & $1.0 \mathrm{E}+02$ \\
\hline${ }^{244} \mathrm{Cm}$ & $9.2 \mathrm{E}+02$ & $9.5 \mathrm{E}+02$ & $1.1 \mathrm{E}+02$ & $9.0 \mathrm{E}+01$ & $1.4 \mathrm{E}+03$ & $1.2 \mathrm{E}+00$ & $1.0 \mathrm{E}+02$ \\
\hline
\end{tabular}

${ }^{a}$ A 5:1 ratio acid to sludge was used for the tests. Mixtures were shaken, centrifuged and the phases separated for analysis. Actual acid concentrations were $3.97 M$ and $8.03 M$ respectively.

${ }^{b} \mathrm{ND}$ indicates that the analyte was below the detectable limit of the instrument in the dilution analyzed. 
Table 4. Sample analysis data summary for GAAT W-9 transfers to the MVSTs

\begin{tabular}{|c|c|c|c|c|c|c|c|}
\hline $\begin{array}{c}\text { Transfer } \\
\text { date }\end{array}$ & $\begin{array}{c}\text { TSS } \\
(\mathrm{mg} / \mathrm{L})\end{array}$ & $\begin{array}{c}\text { TSS50 } \\
(\mathrm{mg} / \mathrm{L})\end{array}$ & $\begin{array}{l}\text { TSS100 } \\
\text { (mg/L) }\end{array}$ & $\begin{array}{c}\text { Total } \\
\text { solids } \\
\text { (mg/kg) }\end{array}$ & $\begin{array}{c}\text { Type } \\
\text { of } \\
\text { mixer }\end{array}$ & pH & $\begin{array}{l}\text { Density } \\
\text { (g/mL) }\end{array}$ \\
\hline $5 / 25 / 99$ & 12,000 & $<10$ & ND & 28,200 & PulsAir & ND & 1.024 \\
\hline $6 / 11 / 99$ & 18,700 & $\mathrm{ND}^{a}$ & ND & 38,000 & PulsAir & ND & 1.037 \\
\hline $6 / 22 / 99$ & 35,900 & ND & ND & 55,500 & PulsAir & ND & 1.044 \\
\hline $7 / 22 / 99$ & 39,800 & ND & ND & 55,500 & PulsAir & 9.93 & 1.044 \\
\hline $7 / 28 / 99$ & 33,200 & ND & ND & 52,600 & PulsAir & 9.98 & 1.058 \\
\hline $8 / 17 / 99$ & 30,800 & ND & ND & 49,600 & PulsAir & 9.92 & 1.050 \\
\hline 9/9/99 & 38,400 & ND & ND & 56,100 & PulsAir & 9.92 & 1.023 \\
\hline 9/28/99 & 29,000 & 460 & ND & 48,400 & $\begin{array}{l}\text { PulsAir } \\
\text { Flygt }\end{array}$ & 9.90 & 1.047 \\
\hline $10 / 26 / 99$ & 49,800 & 1000 & ND & 68,400 & $\begin{array}{l}\text { PulsAir } \\
\text { Flygt }\end{array}$ & 9.86 & 1.055 \\
\hline $11 / 17 / 99$ & 39,300 & 860 & ND & 56,400 & $\begin{array}{l}\text { PulsAir } \\
\text { Flygt }\end{array}$ & 9.85 & 1.028 \\
\hline $12 / 7 / 99$ & 36,400 & 180 & ND & 51,500 & $\begin{array}{l}\text { PulsAir } \\
\text { Flygt }\end{array}$ & 9.77 & 1.024 \\
\hline $1 / 13 / 00$ & 44,500 & 1340 & ND & 58,200 & $\begin{array}{l}\text { PulsAir } \\
\text { Flygt }\end{array}$ & ND & 1.045 \\
\hline $1 / 20 / 00$ & 31,500 & 950 & ND & 31,500 & $\begin{array}{l}\text { PulsAir } \\
\text { Flygt }\end{array}$ & 9.75 & 1.042 \\
\hline $2 / 11 / 00$ & 41,000 & 950 & ND & 55,800 & $\begin{array}{l}\text { PulsAir } \\
\text { Flygt }\end{array}$ & 9.75 & 1.043 \\
\hline $2 / 18 / 00$ & 35,800 & ND & 350 & 59,600 & $\begin{array}{l}\text { PulsAir } \\
\text { Flygt }\end{array}$ & 9.72 & 1.042 \\
\hline $3 / 9 / 00$ & 23,000 & ND & $130^{b}$ & 39,200 & $\begin{array}{l}\text { PulsAir } \\
\text { Flygt }\end{array}$ & 9.57 & 1.029 \\
\hline $3 / 23 / 00$ & 25,800 & ND & 160 & 49,900 & $\begin{array}{l}\text { PulsAir } \\
\text { Flygt }\end{array}$ & 9.53 & 1.039 \\
\hline $3 / 30 / 00$ & 30,900 & ND & 480 & 54,000 & $\begin{array}{l}\text { PulsAir } \\
\text { Flygt }\end{array}$ & 9.54 & 1.049 \\
\hline
\end{tabular}

${ }^{a} \mathrm{ND}=$ no data.

${ }^{b}$ Sample collected on 3/15/00. 
Table 5. Sample analysis data collected during transfer operations from GAAT W-9 to the MVSTs

\begin{tabular}{|c|c|c|c|c|c|c|c|c|c|}
\hline $\begin{array}{l}\text { Transfer } \\
\text { number }\end{array}$ & $\begin{array}{l}\text { Transfer } \\
\text { date }\end{array}$ & $\begin{array}{c}\text { Total } \\
\text { solids } \\
\left(10^{4} \mathrm{mg} / \mathrm{L}\right)\end{array}$ & $\begin{array}{c}\text { TSS } \\
\left(10^{4} \mathrm{mg} / \mathrm{L}\right)\end{array}$ & $\begin{array}{l}\text { Density } \\
(\mathrm{g} / \mathrm{mL})\end{array}$ & $\begin{array}{l}\text { Volume } \\
\text { (gal) }\end{array}$ & $\begin{array}{l}\text { Total activity } \\
\left(10^{6} \mathrm{~Bq} / \mathrm{mL}\right)\end{array}$ & $\begin{array}{c}\text { Solids } \\
\text { transferred }^{a} \\
\text { (g) }\end{array}$ & $\begin{array}{c}\text { Sludge } \\
\text { transferred }^{b} \\
(\text { gal })\end{array}$ & $\begin{array}{l}\text { Total activity } \\
\text { transferred }^{c} \\
\text { (Ci) }\end{array}$ \\
\hline 1 & $5 / 25 / 99$ & 2.82 & 1.20 & 1.024 & 10,435 & 0.37 & $1,113,801$ & 753 & 395 \\
\hline 2 & $6 / 11 / 99$ & 3.80 & 1.87 & 1.037 & 25,704 & 1.17 & $3,697,006$ & 2,501 & 3,074 \\
\hline 3 & $6 / 22 / 99$ & 5.55 & 3.59 & 1.044 & 23,795 & 1.17 & $4,998,556$ & 3,381 & 2,845 \\
\hline 4 & $7 / 22 / 99$ & 5.55 & 3.98 & 1.044 & 27,220 & 2.05 & $5,718,037$ & 3,868 & 5,703 \\
\hline 5 & $7 / 28 / 99$ & 5.26 & 3.32 & 1.058 & 26,144 & 1.95 & $5,205,035$ & 3,521 & 5,210 \\
\hline 6 & $8 / 17 / 99$ & 4.96 & 3.08 & 1.050 & 41,860 & 1.30 & $7,858,629$ & 5,316 & 5,562 \\
\hline 7 & 9/9/99 & 5.61 & 3.84 & 1.023 & 37,570 & 1.66 & $7,977,557$ & 5,396 & 6,374 \\
\hline 8 & $9 / 28 / 99$ & 4.84 & 2.90 & 1.047 & 29,813 & 0.92 & $5,461,563$ & 3,694 & 2,803 \\
\hline 9 & $10 / 26 / 99$ & 6.84 & 4.98 & 1.055 & 25,100 & 1.40 & $6,498,239$ & 4,395 & 3,591 \\
\hline 10 & $11 / 17 / 99$ & 5.64 & 3.93 & 1.028 & 25,330 & 0.92 & $5,407,296$ & 3,657 & 2,382 \\
\hline 11 & $12 / 7 / 99$ & 5.15 & 3.64 & 1.024 & 36,659 & 0.75 & $7,145,847$ & 4,833 & 2,810 \\
\hline 12 & $1 / 13 / 00$ & 5.82 & 4.45 & 1.045 & 23,048 & 0.98 & $5,077,175$ & 3,434 & 2,308 \\
\hline $13^{d}$ & $1 / 20 / 00$ & 3.15 & 3.15 & 1.042 & 24,300 & 0.73 & $3,725,008$ & 2,520 & 1,813 \\
\hline 14 & $2 / 11 / 00$ & 5.58 & 4.10 & 1.043 & 21,000 & 0.95 & $4,435,263$ & 3,000 & 2,039 \\
\hline $15^{d}$ & $2 / 18 / 00$ & 5.96 & 3.58 & 1.042 & 24,027 & 0.75 & $4,074,210$ & 2,756 & 1,842 \\
\hline $16^{d}$ & $3 / 9 / 00$ & 3.92 & 2.30 & 1.029 & 27,120 & 0.48 & $3,284,774$ & 2,222 & 1,330 \\
\hline $17^{d}$ & $3 / 23 / 00$ & 4.99 & 2.58 & 1.039 & 25,066 & 0.57 & $3,301,643$ & 2,233 & 1,460 \\
\hline $18^{d}$ & $3 / 30 / 00$ & 5.40 & 3.09 & 1.049 & 29,086 & 0.68 & $4,392,611$ & 2,971 & 2,021 \\
\hline Total & & & & & 483,277 & & $89,372,250$ & 60,451 & 53,562 \\
\hline
\end{tabular}

a "Solids transferred" is calculated from: Volume $\times 3.785 \mathrm{~L} / \mathrm{gal} \times$ Total solids $\times 10^{-3} \mathrm{~g} / \mathrm{mg}$.

$b$ "Sludge transferred" is an estimate of sludge removed from the GAATs and is based on 88,000 gal of wet sludge estimated to initially be in GAATs at an average bulk density of $1.26 \mathrm{~g} / \mathrm{mL}$ and solids content of $31 \%$ (based on Remedial Investigation data) and is calculated from:

- Total solids in GAATs sludge $=88,000 \mathrm{gal} \times 1.26 \mathrm{~g} / \mathrm{mL} \times 10^{3} \mathrm{~mL} / \mathrm{L} \times 3.785 \mathrm{~L} / \mathrm{gal} \times 0.31=1.301 \times 10^{8} \mathrm{~g}$

- Gallons of sludge transferred $=$ Solids Transferred $\div\left(1.301 \times 10^{8} \mathrm{~g}\right) \times 88,000 \mathrm{gal}$

$c$ "Total activity transferred" is an estimate of the total curies transferred to the MVSTs and is calculated from:

- Total activity in $\mathrm{Bq} / \mathrm{ml} \times 103 \mathrm{~mL} / \mathrm{L} \times 3.785 \mathrm{~L} / \mathrm{gal} \times$ Volume transferred in gal $\times 2.7 \times 10^{-11} \mathrm{Ci} / \mathrm{Bq}$

${ }^{d}$ Transfers utilized recycled supernate - total solids is assumed to be the suspended solids plus $50 \%$ of the dissolved solids $(0.9 \times 104$ mg/L) to reflect the mixing of the recycled supernate with the dissolved solids from $\sim 4 \mathrm{ft}$ of liquid inventory remaining in W-9 before supernate recycle was initiated. 
ORNL Photo-6341-2000

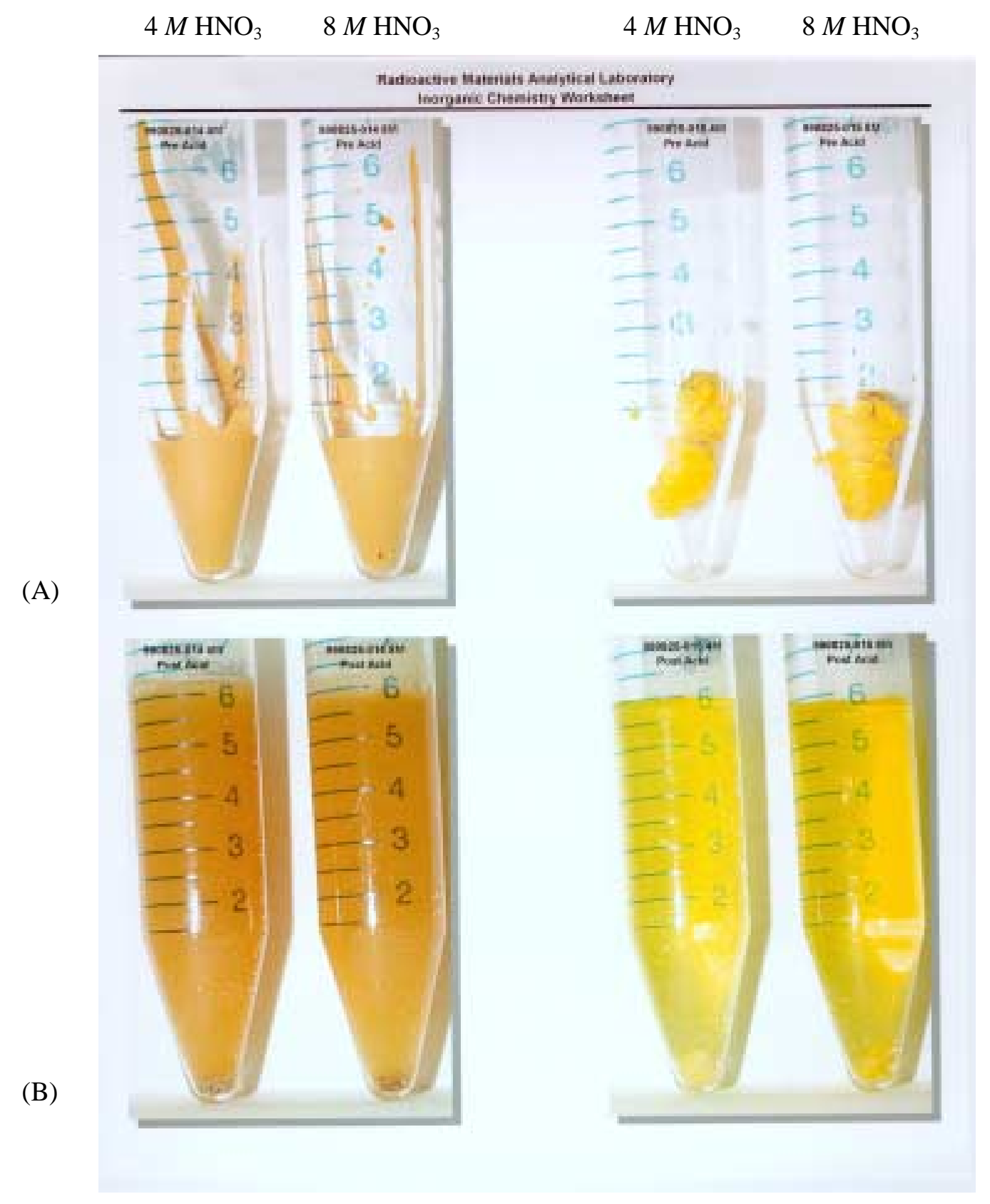

Fig. 10. W-9 supernatant and sludge samples (A) before acid digestion and (B) after acid digestion. 


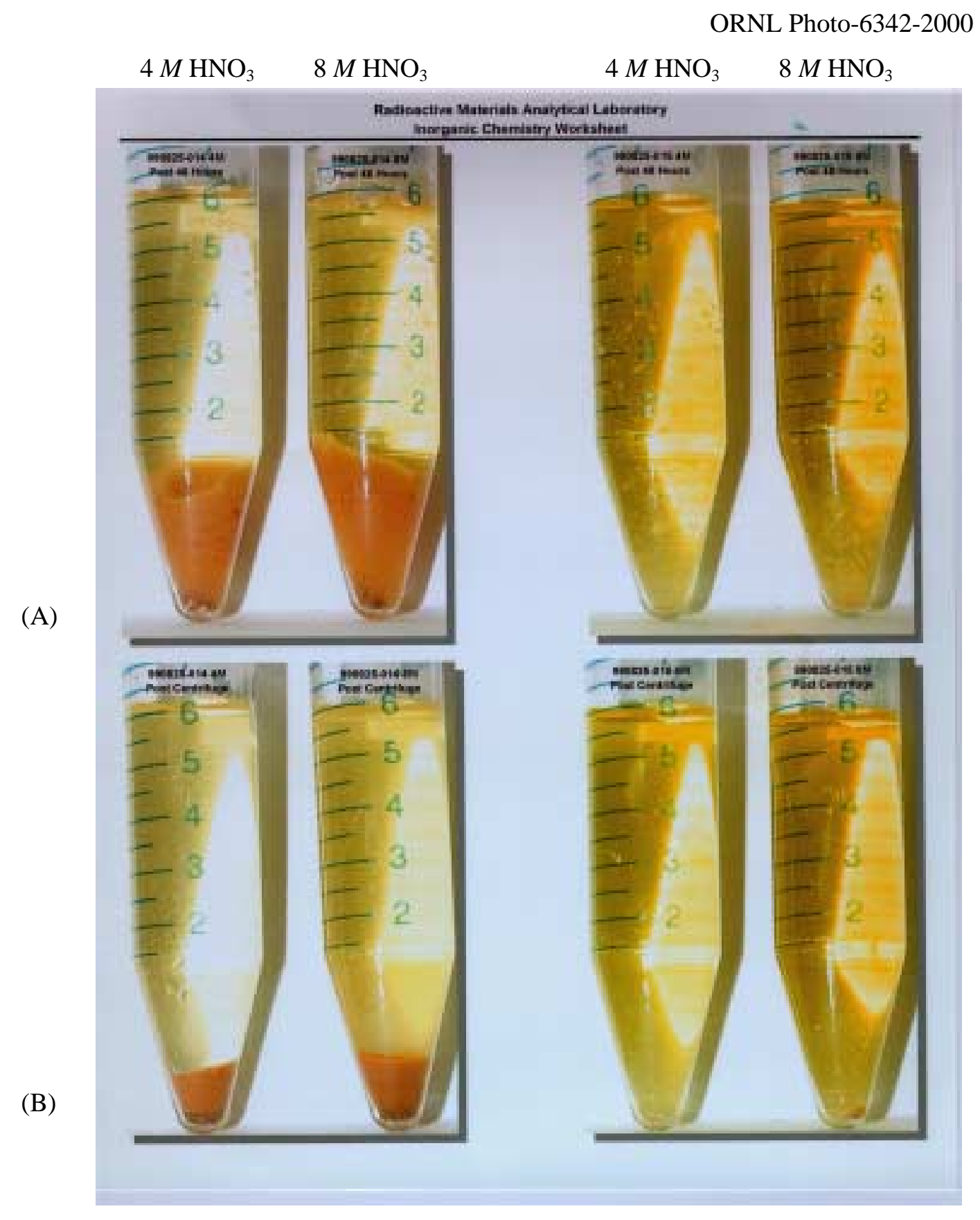

Fig. 11. W-9 supernatant and sludge samples (A) after acid digestion and settling and (B) after centrifugation. 


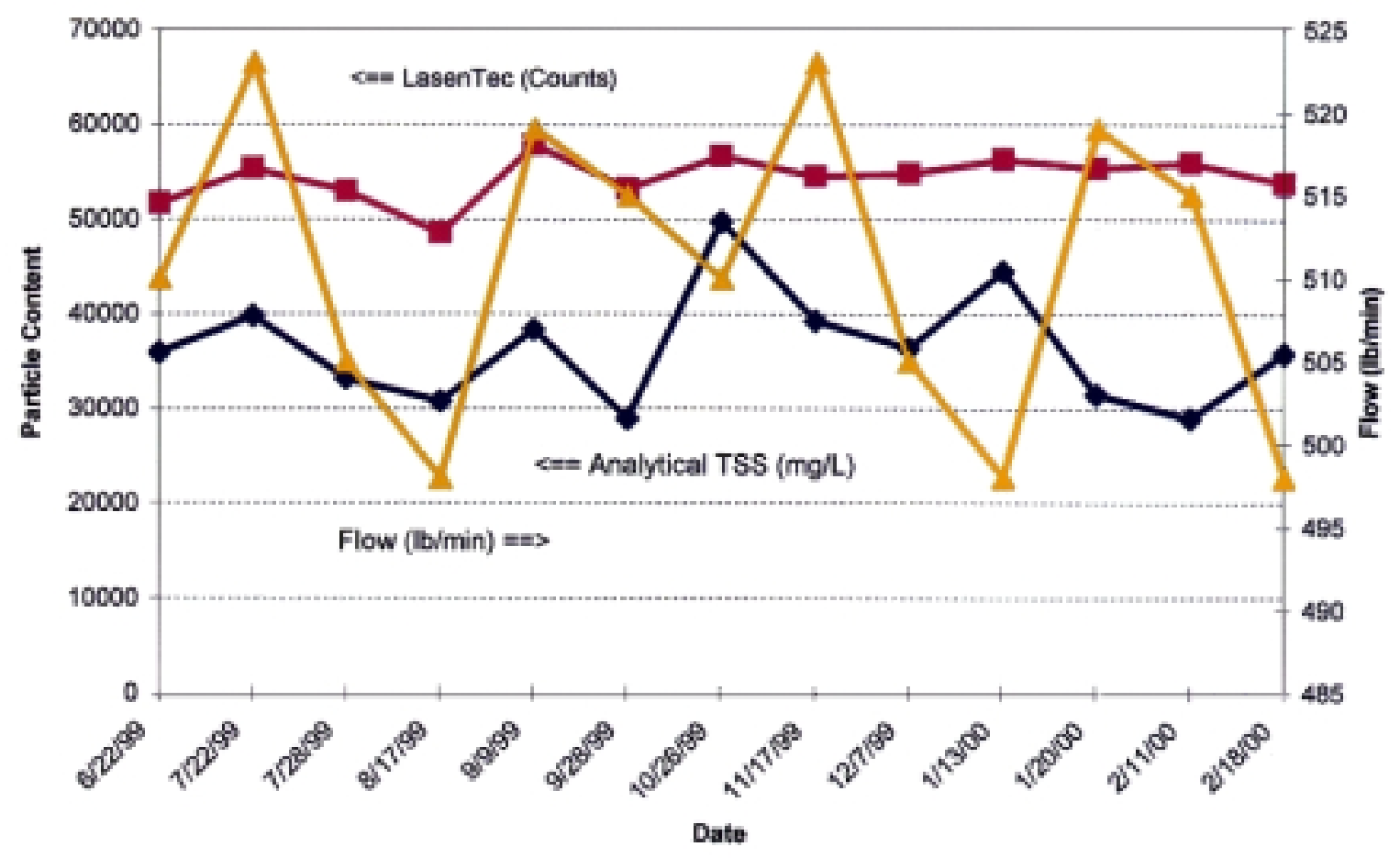

Fig. 12. Comparison of LasenTec and analytical data on the sludge samples from GAAT W-9.

\section{WASTE-RETRIEVAL OPTIONS FOR GAAT W-9}

The GAATs are located in the center of the main ORNL complex. The location of the GAAT tank farm relative to the BVESTs is illustrated in Fig. 13. The inlet to BVEST W-23 is $\sim 500 \mathrm{ft}$ from GAAT W-9 and is $\sim 5 \mathrm{ft}$ above the discharge point from W-9. The SCS has been used to remove the majority of the waste transferred to GAAT W-9 during the efforts to consolidate the wastes from the other GAATs. However, it is anticipated that a portion of the waste remaining in W-9 must be mobilized and mixed using more aggressive means than provided by the SCS.

The wastes in W-9 can be classified as either pumpable or nonpumpable. Some of the nonpumpable material may be conditioned and converted into pumpable material. The majority of waste present is considered to be pumpable wastes. Pumpable wastes are wastes that can be easily retrieved and transferred through the existing waste transfer lines without a significant risk of blockage. In general these wastes consist of slurries with particles $<100 \mu \mathrm{m}$ in diameter and $<5$ to $10 \mathrm{wt} \%$ solids concentration. Higher TSS contents are allowed if data show that settling will not be a problem during the transfer operation. The nonpumpable waste includes debris, hard sludge, agglomerations, and flowable material that may be difficult to retrieve due to its location or shallow depth. Any nonpumpable residual materials remaining after the bulk waste removal operations are completed will be characterized, and a decision will be made on the necessity of any further clean-up or waste removal. The options considered included the use of new equipment as well as reuse of the existing GAAT transfer and retrieval systems wherever practical. In actual practice, pumpable material will be segregated from nonpumpable material by retrieving the pumpable material using the current pumping-based methods. 


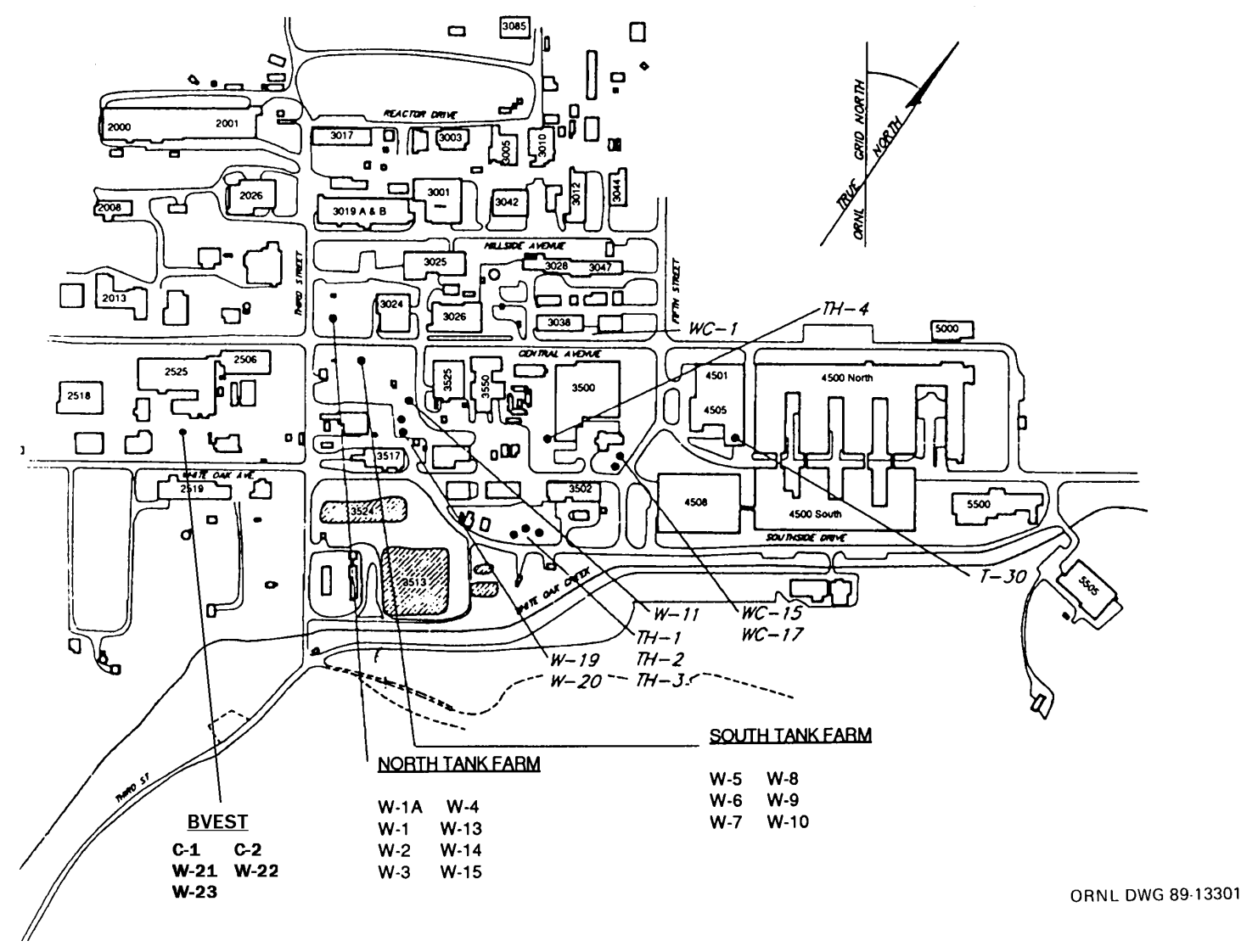

Fig. 13. Location of GAATs STF relative to the BVESTs.

\subsection{ASSUMPTIONS}

The following assumptions were used in the evaluation of GAAT W-9 waste-transfer and -retrieval system options:

1. An adequate supply of clean process water is available in the STF.

2. An adequate source of supernate will be available in the STF.

3. The slurry being transferred is such that it will tolerate flow interruptions of up to $15 \mathrm{~min}$ without settling in quantities that result in blockage of the transfer line.

4. In the event of a power outage or other failure of the transfer system, sufficient quantities of supernate or clean process water can be used such as to flush the transfer line within 15 min.

5. The existing CSEE and jet pump are adequate for converting some of the nonpumpable waste to pumpable slurry and removing the waste from W-9. This retrieval system represents the baseline system for comparison of the other options.

6. The properties of the waste present in W-9 are consistent with waste properties experienced in North Tank Farm (NTF) and STF tanks. 
7. BVEST W-23 will be used as an intermediate batch tank to prevent slurries with excessive solids concentrations from entering the transfer line to the MVSTs. This minimizes the potential for blockage of ORNL's primary waste transfer line.

\subsection{FUNCTIONS AND REQUIREMENTS}

The waste-retrieval and -transfer system for GAAT W-9 was required to have the following functions and requirements:

1. Provide a throughput of 50-60 gal $/ \mathrm{min}$. Flow rates must not exceed $100 \mathrm{gal} / \mathrm{min}$.

2. Provide adequate head to facilitate transfer from GAAT W-9 to BVEST W-23. Tank $\mathrm{W}-9$ in the STF is located $\sim 500 \mathrm{ft}$ from W-23. W-23 is west of tank W-9. The inlet to tank W-23 is $\sim 5 \mathrm{ft}$ higher than the opening in tank W-9.

3. Provide variable operation (i.e., speed control) to accommodate differing flow rate, discharge head, and waste properties.

4. Provide filtering, grinding, or other means of solids classification to ensure that solids larger than $100 \mu \mathrm{m}$ are not transferred from W-9 to W-23.

5. Withstand and provide reliable containment for tank waste.

6. Provide a rinse-back-flush capability for all components of the system that are used to pump or transfer waste.

7. Provide a positive means of preventing contamination of back-flush-rinsing supply and other process water.

8. No discharge of gas, vapor, liquid, or solids from the tank to the external environment.

9. Ensure that the maximum airflow into the tank, if applicable, does not produce a positive pressure inside the tank or riser space. The maximum allowable airflow rate into the source tank (W-9) is $200 \mathrm{ft}^{3} / \mathrm{min}$.

10. Provide for control and monitoring of the system.

11. Operate in an outdoor installation, which is exposed to full sun and weather. Ambient conditions range from 0 to $120^{\circ} \mathrm{F}$ and $100 \%$ relative humidity.

12. Withstand storage at ambient conditions of -20 to $120^{\circ} \mathrm{F}$ and $100 \%$ relative humidity, condensing.

13. Not exceed a load limit of $2,000 \mathrm{lb}$ on the tank riser. Total weight of all associated equipment must be provided to ORNL Engineering, which will evaluate platform loading prior to installation.

\subsection{DESCRIPTION OF GAAT W-9 WASTE-RETRIEVAL AND -TRANSFER SYSTEM OPTIONS FOR PUMPABLE WASTES}

A variety of bulk waste-retrieval and -transfer options have been considered for the pumpable wastes present in GAAT W-9. The initial set of options included the following:

1. Tank in a tank to act as an accumulator tank for the transfer to MVSTs;

2. Additional mixers (Russian Pulsating Mixer, Flygt, PulsAir) to mobilize the waste for the transfer to the MVSTs;

3. Waste container/cask and truck transport to the MVSTs;

4. ESG TracPump ${ }^{\mathrm{TM}}$ to retrieve and transfer waste from W-9 to the BVESTs;

5. Pit Hog ${ }^{\mathrm{TM}}$ type chopper pump for retrieval and transfer of waste to the BVESTs;

6. ESG Centrifugal Pump with a grade-level transfer line; and 
7. Modify or add to the existing STF Tank Waste Retrieval System (TWRS) (i.e., MLDUA, Houdini Vehicle, CSEE, Jet Pump, Flow Control Equipment-Containment Box (FCE$\mathrm{CB}$ ), associated WD\&CS pumps, etc, ) to transfer waste from W-9 to the BVESTs followed by use of the Atomic Energy Authority Technology (AEAT) Pulsed Jet Fluidic Mixing Technology and existing transfer pumps to transfer the waste to the MVSTs.

The options were reviewed, and option 1 was selected for further evaluation. Option 1 uses the existing TWRS to transfer the waste to the BVESTs and then uses the AEAT Pulsed Jet Fluidic Mixing system and existing Moyno ${ }^{\mathrm{TM}}$ pumps to transfer the waste to the MVSTs. This approach offers a significant advantage in maximizing the use of existing hardware, thereby reducing risk, and cost associated with designing, developing, fabricating, testing, deploying, operator-training and using new equipment. The alternatives listed below, provide variations on retrieval, transfer, holding tank, and conditioning systems for option 7.

1. TWRS with two jet pumps and overhead transfer line for gravity feed to BVESTs;

2. TWRS with two jet pumps, SCS, and overhead transfer line for gravity feed to the BVESTs;

3. TWRS with one jet pump, slurry consolidation tank, DiscFlo pump, SCS, and overhead transfer line for gravity feed to BVESTs;

4. TWRS with one jet pump, slurry consolidation tank, DiscFlo pump, SCS, and grade-level transfer line to BVESTs; and

5. TWRS with one jet pump, slurry consolidation tank, booster pump, SCS, and grade-level transfer line to BVESTs.

The primary advantages and disadvantages of these options and alternatives are described in Tables 7 and 8. Sections 3.3.1-3.3.12 provide brief descriptions of each of the options and alternatives considered for the W-9 waste-transfer operation for the pumpable wastes. 
Table 6. Advantages and disadvantages of the primary waste-retrieval and transfer options for pumpable wastes

\begin{tabular}{|c|c|c|}
\hline Option & Advantages & Disadvantages \\
\hline 1. Tank in tank & $\begin{array}{l}\text { - } \begin{array}{l}\text { Ability to control "batch" } \\
\text { characteristics }\end{array} \\
\text { - No LLLW }{ }^{a} \text { impacts } \\
\text { - } \\
\text { Allows use of other GAATs } \\
\text { for secondary containment }\end{array}$ & $\begin{array}{ll}\text { - } & \text { Need design-No FY } 1999 \text { funds available } \\
\text { - } & \$ 0.5 \mathrm{M}-\$ 1 \mathrm{M} \text { Capital investment } \\
\text { - } & \text { Winter construction-Needed by spring } \\
& \text { FY } 2000 \\
\text { - } & \text { Schedule impacts } \\
\text { - } & \text { New system startup challenges } \\
\text { - } & \text { Waste in clean tank } \\
\text { - } & \text { Smaller batches to MVSTs based on } \\
& \text { operating characteristics of TWRS and } \\
& \text { tank size } \\
\text { - } & \text { Possible removal of tank dome } \\
\text { - } & \text { Additional deactivation \& } \\
& \text { decommissioning (D\&D) } \\
\end{array}$ \\
\hline $\begin{array}{l}\text { 2. Use additional } \\
\text { existing mixers } \\
\text { (Russian, Flygt, } \\
\text { PulsAir) }\end{array}$ & 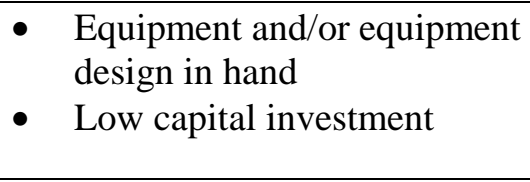 & $\begin{array}{l}\text { - } \quad \text { Uncertain performance } \\
\text { - } \quad \text { Likely to leave significant residual } \\
\text { - } \quad \text { More equipment moves }\end{array}$ \\
\hline $\begin{array}{l}\text { 3. Cask / truck } \\
\text { transport }\end{array}$ & 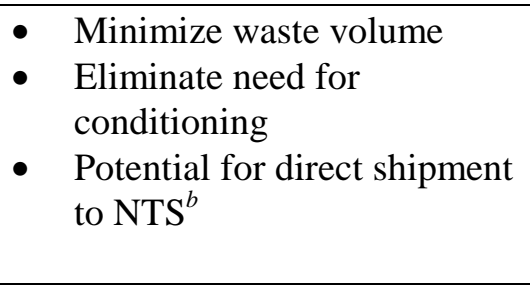 & $\begin{array}{ll}\text { - } & \text { Transportation } \\
\text { - } & \text { Cask unloading at MVSTs } \\
\text { - } & \text { Potential impact to sludge removal from } \\
& \text { MVSTs } \\
\text { - } & \text { Safety/environmental documentation } \\
\text { - } & \text { Cask cost } \\
\end{array}$ \\
\hline 4. ESG Trac pump & $\begin{array}{l}\text { - } \begin{array}{l}\text { May eliminate the need for a } \\
\text { booster pump } \\
\text { - } \\
\text { Equipment available from } \\
\text { another DOE Program }\end{array} \\
\end{array}$ & $\begin{array}{ll}\text { - } & \text { Requires additional development and } \\
\text { testing }\end{array}$ \\
\hline 5. Pit Hog pump & $\begin{array}{l}\text { - May eliminate the need for a } \\
\text { booster pump } \\
\text { - Low cost }\end{array}$ & $\begin{array}{l}\text { - Flooded suction line results in incomplete } \\
\text { inefficient operation } \\
\text { Requires additional design, development, } \\
\text { and testing }\end{array}$ \\
\hline $\begin{array}{l}\text { 6. ESG centrifugal } \\
\text { pump }\end{array}$ & $\begin{array}{l}\text { - No booster pump required if } \\
\text { SCS used } \\
\text { - Low cost }\end{array}$ & $\begin{array}{l}\text { - } \quad \text { Requires modifications } \\
\text { - } \text { Too heavy for MLDUA } \\
\text { - } \quad \text { May require intermediate storage tank } \\
\text { - } \quad \text { Flooded suction line results in incomplete } \\
\text { cleaning inefficient operation }\end{array}$ \\
\hline $\begin{array}{l}\text { 7. Use TWRS to } \\
\text { pump tank W-9 } \\
\text { to W-23; use } \\
\text { AEAT } \\
\text { technology to } \\
\text { mix and pump } \\
\text { waste to MVSTs }\end{array}$ & $\begin{array}{ll}\text { - } & \text { TWRS, SCS and AEAT } \\
\text { - } & \text { TWoven technology } \\
\text { TWR, SCS and AEAT } \\
\text { equipment in-hand } \\
\text { - } & \text { Minimal capital investment } \\
\text { - } & \text { Minimum schedule impact } \\
\text { - } & \text { May eliminate need for } \\
& \text { grinders } \\
\end{array}$ & $\begin{array}{ll}\text { - } & \text { Requires booster pump } \\
\text { - } & \text { Difficult valve-box modification } \\
\text { - } & \text { Potential water balance and LLLW } \\
\text { - } & \text { operations impact } \\
\text { - } & \text { Potential need for WAC waiver } \\
\text { - } & \text { May use previously cleaned inactive } \\
& \text { GAAT for supernate recycle } \\
\end{array}$ \\
\hline
\end{tabular}


Table 7. Advantages and disadvantages of the alternatives for the down selected waste retrieval and transfer option for GAAT W-9 pumpable wastes

\begin{tabular}{|c|c|c|}
\hline Alternative & Advantages & Disadvantages \\
\hline $\begin{array}{l}\text { 1. TWRS with two jet } \\
\text { pumps and overhead } \\
\text { transfer line for } \\
\text { gravity feed to } \\
\text { BVESTs }\end{array}$ & $\begin{array}{l}\text { - } \\
\text { transfer line } \\
\text { - } \quad \text { Low-pressure transfer } \\
\text { - } \quad \text { Low probability of } \\
\text { clogging } \\
\text { - } \quad \text { Lowest cost }\end{array}$ & $\begin{array}{l}\text { Overhead radioactive waste transfer } \\
\text { line } \\
\text { - Transfer line protection, shielding, } \\
\text { design considerations } \\
\text { - Two-stage transfer } \\
\text { - No control of particle size to } \\
\text { BVESTs }\end{array}$ \\
\hline $\begin{array}{l}\text { 2. TWRS with two jet } \\
\text { pumps, SCS, and } \\
\text { overhead transfer line } \\
\text { for gravity feed to } \\
\text { BVESTs }\end{array}$ & $\begin{array}{ll}\text { - } & \text { No excavation for } \\
\text { transfer line } \\
\text { - } & \text { Low-pressure transfer } \\
\text { - } & \text { Control of particles } \\
\text { size to BVESTs } \\
\text { - } \quad \text { Low probability of } \\
\text { clogging } \\
\text { - Small incremental } \\
\text { cost }\end{array}$ & $\begin{array}{ll}\text { - } & \text { Overhead radioactive waste transfer } \\
\text { line }\end{array}$ \\
\hline $\begin{array}{l}\text { 3. TWRS with one jet } \\
\text { pump, slurry } \\
\text { consolidation tank, } \\
\text { DiscFlo pump, SCS, } \\
\text { and overhead transfer } \\
\text { line for gravity feed } \\
\text { to BVESTs }\end{array}$ & $\begin{array}{ll} & \text { No excavation for } \\
\text { transfer line } \\
\text { - } \\
\text { Reduced waste } \\
\text { inventory in transfer } \\
\text { line } \\
\text { - Control of particles } \\
\text { size to BVESTs } \\
\text { - Low probability of } \\
\text { clogging }\end{array}$ & $\begin{array}{l}\text { - } \text { Overhead radioactive waste transfer } \\
\text { line } \\
\text { - } \quad \text { Possible transfer line pressurization } \\
\text { by DiscFlo pump } \\
\text { - } \quad \text { Transfer line protection, shielding, } \\
\text { and design considerations } \\
\text { - } \quad \text { Requires consolidation tank } \\
\text { - } \quad \text { DiscFlo pump reliability } \\
\text { - Increased complexity and cost }\end{array}$ \\
\hline $\begin{array}{l}\text { 4. TWRS with one jet } \\
\text { pump, slurry } \\
\text { consolidation tank, } \\
\text { DiscFlo pump, SCS, } \\
\text { and grade-level } \\
\text { transfer line to } \\
\text { BVESTs }\end{array}$ & $\begin{array}{ll}\text { - } & \text { Protected transfer line } \\
\text { - } & \text { Control of particles } \\
\text { size to BVESTs } \\
\text { - } & \text { Maximum use of } \\
\text { existing system } \\
\text { configuration }\end{array}$ & $\begin{array}{ll}\text { - } & \text { Excavation for transfer line to } \\
& \text { BVESTs } \\
\text { - } & \text { Requires consolidation tank } \\
\text { - } & \text { DiscFlo pump reliability } \\
\text { - Increased complexity and cost }\end{array}$ \\
\hline $\begin{array}{l}\text { 5. TWRS with one jet } \\
\text { pump, slurry } \\
\text { consolidation tank, } \\
\text { booster pump, SCS, } \\
\text { and grade-level } \\
\text { transfer line to } \\
\text { BVESTs }\end{array}$ & $\begin{array}{ll}\text { - } & \text { Protected transfer line } \\
\text { - } & \text { Control of particles } \\
& \text { size to BVESTs } \\
\text { - } & \text { Improved reliability }\end{array}$ & $\begin{array}{ll}\text { - } & \text { Excavation for transfer line to } \\
& \text { BVESTs } \\
\text { - } & \text { Requires consolidation tank } \\
\text { - } & \text { Requires modification to SCS } \\
\text { - } & \text { Requires supernate pumping system } \\
\text { or additional process water }\end{array}$ \\
\hline
\end{tabular}




\subsubsection{Tank in Tank}

Using this method would be similar to the method used to clean the other Gunite tanks in the STF. The MLDUA, HMA, and the Houdini Vehicle would be moved to a new platform above tank W-9, where the MLDUA would be used initially to clean one quadrant and then progress to each of the remaining quadrants as the waste is removed. The GSEE would be used to clean the tank walls, and the HMA and CSEE would be used to remove the residual waste material.

Because the discharge head of the HMA jet pump is insufficient to pump the material to the MVSTs, the discharge of the HMA jet pump would be directed to an accumulator tank with a capacity of $\sim 10,000$ to 20,000 gal. The accumulator tank would be located inside tank W-10 and would be inserted through a riser. The existing DiscFlo pump would be installed within the small tank along with a mixing system to maintain the solids in suspension and transfer the waste to the MVSTs.

Various tank-in-tank options have been considered to-date. These options include:

- Open roof of GAAT W-10 and insert a prefabricated tank

- Install a single membrane thermo-set blowup tank

- Install a double membrane blowup tank with resin filling the space between the two membranes

Installing the accumulator tank inside waste-consolidation tank W-9 was also considered. However, this alternative was eliminated due to concerns over placing the tank in contact with the waste, limitations on space, and interference with the waste-retrieval operations.

The tank-in-tank option is anticipated to require a significant capital investment and long leadtime to design, fabricate, and install. This option requires the installation of an accumulator tank inside either a previously cleaned and empty tank (W-10) or a tank with significant amounts of sludge present (W-9). The high anticipated costs and potential impacts on the schedule have eliminated this option from further consideration.

\subsubsection{Additional Mixers (e.g., Russian Pulsating Mixer, Flygt, PulsAir)}

This method would be similar to the approaches used in tank W-9 for the waste transfers to the MVSTs. The goal of this approach would be to add additional mixing technologies, which would increase the suspension of sludge in the supernate. This approach would be based on no requirement to wash the walls, or would require extensive equipment relocation. Uncertainty over the performance of these mixer systems in the retrieval of the residual sludge from W- 9 and the possibility of a potentially large heel of waste being left behind eliminated this option from further consideration.

\subsubsection{Waste Container/Cask and Truck Transport to MVSTs}

With this approach the sludge remaining in tank W-9 would be removed using the MLDUA, Houdini Vehicle, and HMA and transferred to a cask for transport over the roads to the MVSTs. Once the cask arrives at the MVSTs it would then be unloaded and reused for transport of the remaining sludge in $\mathrm{W}-9$. Transportation, handling, and safety concerns associated with the 
movement of radioactive sludges via the roadways to the MVSTs eliminated this option from further consideration.

\subsubsection{ESG TracPump for Retrieving and Transferring Waste to the BVESTs}

ESG Manufacturing Technologies teamed with Framatome Technologies and Los Alamos Technical Associates to propose and subsequently win the Hanford contract award for the cleanup of Hanford's C-106 tank. The basis of the proposal was ESG's TracPump. The TracPump is a tracked remotely operated vehicle with an on-board pump, which has proven successful in the oil refinery business in removing sludge from tanks. The Hanford Tanks Initiative Project was shut down in FY 1999 for lack of funding, and the vehicle was never completed. Although, this vehicle may be available along with possible funding from Hanford to "cold" test at ORNL before its deployment at Hanford, the level of uncertainty over the performance of the system and the tight deployment schedule eliminated this option from further consideration. For this option, the TracPump would be used to retrieve and transfer waste from W-9 to the BVESTs. The existing AEAT Pulsed Jet Fluidic Mixer and Moyno pumps would be used to mobilize and transfer the waste to the MVSTs.

\subsubsection{Pit Hog for Retrieving and Transferring Waste to the BVESTs}

The Pit Hog is a chopper pump manufactured by Liquid Waste Technology Inc. that can be used to break apart large-diameter solids. This type of pump has been considered at various times throughout the GAATs Remediation Project. The concept would be for the MLDUA to grasp the Pit Hog and maneuver the suction around inside the tank so as to remove the sludge. The Pit Hog would conceivably eliminate the need for a booster pump system to transfer the material to the BVESTs. The existing AEAT Pulsed Jet Fluidic Mixer and Moyno pumps would be used to mobilize and transfer the waste from BVEST W-23 to the MVSTs. The Pit Hog pump has never been fully evaluated by the GAATs Remediation Project, so its advantages and disadvantages are not fully known. Therefore, uncertainty over the performance of the system and anticipation of the need for potentially extensive cold testing and development eliminated this option from further consideration. The Pit Hog is a low-efficiency centrifugal pump; which is fabricated from welded steel, making it tolerant of impact. It requires a fully flooded suction inlet and is likely prone to vortex formation if operated near a liquid surface. Therefore, it may be useful for retrieving lumpy sludges under a cover of supernate, but it would probably not be useful for retrieval of the last foot or so of liquid/slurry.

\subsubsection{ESG Centrifugal Pump with Grade-Level Transfer Line}

ESG Inc. manufactures a variety of sludge transfer pumps in addition to the TracPump. An ESG centrifugal pump was considered for use to retrieve and transfer sludge from GAAT tank W-9 to tank BVEST W-23. Performance data and design information on the pump were obtained from ESG, Inc. The off-the-shelf device could be modified to meet the GAATs requirements; however, the unit does not provide any necessary advantages over the existing CSEE for the application under consideration.

ESG pump model 3-18x2 weighs $\sim 75 \mathrm{lb}$ and can generate $300 \mathrm{ft}$ of head at flow rates up to 450 $\mathrm{gal} / \mathrm{min}$. The horsepower needed for this performance requires an oil-based, hydraulic motor. Two modifications that were under consideration were (1) building the pump out of a material lighter than steel to reduce the weight, and (2) changing the hydraulic motor to a water-based motor. ESG was already considering designing a pump made of plastic for Hanford, and the 
company asserted that an aluminum design was possible—although the Hanford chemicals were not compatible with aluminum. A 10-hp Danfoss-Nessie ${ }^{\mathrm{TM}}$ water-based drive motor was located that would have made it possible to operate the drive motor and ESG pump using the water for the CSEE. Under this arrangement, the water discharged from the Danfoss-Nessie motor could be used to feed the CSEE spray nozzles rather than being circulated back to the water supply pump. However, this would require operating the motor at well over 4,000 psi or using larger nozzles in the CSEE. The Danfoss-Nessie drive motor is rated for only 2,000 psi and requires about $13-\mathrm{gal} / \mathrm{min}$ water flow to produce $10 \mathrm{hp}$ at a 2,000-psi pressure drop. Venting the water via the CSEE through the existing nozzles would reduce the available pressure differential and thereby the power output. Some hydraulic motors require close control of the back pressure on the return line. Larger-diameter nozzles on the CSEE may allow this type of operation, but additional development and testing would be required.

The ESG pump does not include spray nozzles, although they could be designed and added to the system. The centrifugal pump is not self-priming. ESG recommends using a jet pump to pull water into the centrifugal pump to prime it.

It was originally thought that the ESG pump could be used to transfer sludge directly from tank W-9 to tank W-23 without the use of an intermediate storage tank. The pump appears to have sufficient power such as to accomplish the transfer and should deliver a reasonably constant flow as long as the suction inlet remains submerged. However, the ESG pump offers no means of "classifying" the waste to ensure that large solids do not enter the transfer line to BVEST W-23. The system would have to be used in conjunction with either an intermediate settling tank or an active classification system such as the SCS.

The ESG pump also has feet, which hold the unit off the floor. The feet on the pump would not allow the retrieval of the last few inches of material from W-9. The CSEE with the jet pump is a true self-priming pump with more desirable overall retrieval properties than the ESG pump for tank waste retrieval operations at ORNL.

Considering the requirements for the delivery of waste to BVEST W-23 it appears that, if the SCS is not used, an intermediate storage tank is essential for use with the ESG pump. Since eliminating this intermediate tank was one of the themes for considering the use of the ESG pump, it was concluded that the use of the existing CSEE and an intermediate tank would be superior to the ESG pump for this particular application.

\subsubsection{Tank Waste-Retrieval System to Transfer Waste From W-9 to the BVESTs and an AEAT Pulsed Jet Fluidic Mixing Technology and Existing Transfer Pumps to Transfer the Waste to the MVSTs}

Using this method would be similar to the method employed to clean the Gunite tanks in the STF. The MLDUA, HMA, and the Houdini Vehicle would be placed on a new platform above tank W-9, where the MLDUA would be used initially to clean one quadrant and then progress to each of the remaining quadrants as the waste is removed. The GSEE would be used to clean the tank walls, and the HMA and CSEE would be used to remove the residual waste material.

Because the discharge head of the HMA jet pump is insufficient to pump the material to the BVESTs, a booster pump in series with the jet pump would be required. A similar booster pump was designed but never used for operations in the NTF. This design can be used as a baseline for the booster pump required in this application. 
Conditioning of waste may include grinding, classification, and/or acid dissolution.

Characterization data from sludge samples taken from W-9 have indicated that a grinder is not likely to be required for the retrieval and relatively short transfer ( $\sim 500 \mathrm{ft})$ of wastes from W-9 to BVEST W-23. Once the waste is in W-23, acid dissolution could be used to significantly reduce the amount of residue and size of particles in the waste. The material in BVEST W-23 will be further conditioned using the AEAT Pulsed Jet Fluidic Mixer system and prepared for transfer to the MVSTs in the same manner as previously described by Kent, et. al. ${ }^{6}$ The existing Moyno pumps would be used to transfer the waste from the BVESTs to the MVSTs.

This option is anticipated to require the use of additional tanks for conditioning and storage of the wastes retrieved from W-9 before these wastes are transferred to the BVESTs and to supply recycled supernate. A small accumulator tank is the preferred option for the conditioned wastes, and one of the clean GAATs is preferred for use as a supernate storage tank. It was initially thought that a waiver from the WACs would be needed for the particle-size constraint for transfers to the MVSTs; however, the results from the analysis of sludge samples from W-9 have shown that a waiver may not be necessary. This option formed the basis of the preferred alternative for waste retrieval and transfer from W-9 to the BVESTs through the use of the existing, proven sludge-removal equipment for cleanout of W-9.

\subsubsection{TWRS with Two Jet Pumps and Overhead Transfer Line for Gravity Feed to the BVESTs}

This option uses the existing CSEE and jet pump to mobilize the sludge from W-9. It is assumed that the MLDUA, WD\&CS, and Houdini Vehicle are used to manipulate and place the CSEE and transfer hoses. In this option, a second jet pump is used to transfer the slurry retrieved from W-9 to the entrance of an elevated gravity feed line to W-23. The second jet pump may not be required for this option, but is included as a booster pump for the primary jet pump used to retrieve the waste from $\mathrm{W}-9$.

Although, the overhead gravity feed line minimizes the head requirements for the pumping system for the transfer to $\mathrm{W}-23$, this concept suffers from the disadvantages of requiring additional shielding, protection, and design scrutiny arising from the implications and potential impacts from failure of the line. The overhead transfer line would be operated at atmospheric pressure and rely on gravity flow from W-9 to W-23. If properly sloped, the overhead transfer line would be tolerant of intermittent flow and generally have a low probability of clogging. This option does not allow for solids classification so it would be possible for solids larger than $100 \mu \mathrm{m}$ to enter the transfer line.

\subsubsection{TWRS with Two Jet Pumps, SCS, and Overhead Transfer Line for Gravity Feed to the BVESTs}

This option is the same as the one described in Sect. 3.3.7.1 but with the addition of the use of the SCS to ensure that only solids less than $100 \mu \mathrm{m}$ are transferred to W-23. In this option, the second jet pump is used to transfer the slurry retrieved from W-9 through the SCS and on to the entrance to an elevated gravity-feed line to W-23. The second jet pump may not have sufficient discharge head such as to force the slurry through the SCS classifiers and on to the entrance of an elevated transfer line to $\mathrm{W}-23$. 
The low point in the transfer line, under this option, occurs in the vicinity of W-9 in a welldefined and relatively small area. Use of the SCS classifier prevents the transfer of solid particles greater than $100 \mu \mathrm{m}$. The major disadvantages of this option are similar to those for the option described in Sect. 3.3.7.1, which are (1) the additional shielding, protection, and design scrutiny from the implications and potential impacts from failure of the overhead waste transfer line, and (2) the necessity of a second jet pump or other type of booster pump to transfer the slurry through the SCS classifiers.

\subsubsection{TWRS with One Jet Pump, Slurry Consolidation Tank, DiscFlo Pump, SCS, and Overhead Transfer Line for Gravity Feed to the BVESTs}

This option uses the existing CSEE and jet pump to mobilize the sludge from W-9 and on to a slurry-consolidation tank, which is anticipated to be installed in W-9. It is assumed that the MLDUA, WD\&CS, and Houdini Vehicle are used to manipulate and place the CSEE and transfer hoses. A slurry-consolidation tank is used as a batch tank for preparing the slurry for transfer to W-23. It is assumed that a small batch tank is located within W-9. The existing DiscFlo pump is then used to transfer the slurry from the consolidation tank through the SCS and on to W-23 through an overhead gravity feed line. The DiscFlo pump is capable of generating in excess of $350 \mathrm{psi}$ of head and has been successfully used to transfer slurries from W-9 through the $\sim 1$-mile long, 2-in.-diam. waste transfer line to the MVSTs. The higher output capacity of the DiscFlo pump (as compared to a jet pump) would allow the use of a smaller-diameter feed line to W-23. Use of smaller 2-in.-diam. line with the DiscFlo pump instead of the 4-in.-diam line assumed for the jet-pump-assisted transfer minimizes the inventory of radioactive material, which would be present in the overhead transfer line, but the smaller line may allow for increased pressurization of the line during transfer.

This option could be implemented with minimal piping changes that would retain the relative design simplicity of the gravity feed line to $\mathrm{W}-23$. However, the option requires the addition of a new batch-transfer tank and relocation of the DiscFlo pump, which is currently installed in W-9. The major disadvantages are (1) the additional shielding, protection, and design scrutiny from the implications and potential impacts from failure of the overhead waste transfer line; (2) the necessity of a batch transfer tank; and (3) reliability concerns with the DiscFlo pump. The DiscFlo pump has been successfully used in the transfer of over 400,000 gal of slurry from W-9 to the MVSTs, but it is thought to be experiencing the initial stages of seal failure.

\subsubsection{TWRS with One Jet Pump, Slurry Consolidation Tank, DiscFlo Pump, SCS, and Grade-Level Transfer Line to the BVESTs}

This option is the same as the option described in Sect. 3.3.7.3 but with the exception of the use of a grade-level waste-transfer line in place of an overhead waste-transfer line. The use of a more traditional transfer-line design is anticipated to be more acceptable from the standpoints of design and safety approval.

One disadvantage of this option is that the use of a grade-level transfer line places a low point in the line near the three-quarter point of the transfer line. The potential for blockage of the line is increased due to the presence of the low point between the feed and discharge points. However, this has been an acceptable risk in past transfers from W-9 to the MVSTs. The 1-mile long, 2-in.-diam MVST transfer line traverses five ridges, has five different low places, and goes under two streams on its way from Bethel Valley to Melton Valley. Other disadvantages of this option 
include (1) the necessity of a batch transfer tank and (2) reliability concerns with the DiscFlo pump.

\subsubsection{TWRS with One Jet Pump, Slurry Consolidation Tank, Booster Pump, SCS, and Grade Level Transfer Line to the BVESTs}

This option is the same as the option described in Sect. 3.3.7.4 but with the exception of the use of a booster pump in place of the DiscFlo Pump and a much simpler and smaller out-of-tank slurry consolidation tank. Placement of the consolidation tank within the riser instead of inside the tank will simplify deployment, reduce cost, and improve the maintainability of the system. A positive displacement, air-diaphragm-type pump would be used to transfer the slurry from the consolidation tank through the SCS and on to W-23. This option would require modifying the SCS piping to install the new booster pump for the transfer of the wastes to W-23.

\subsection{DESCRIPTION OF GAAT W-9 WASTE-RETRIEVAL AND -TRANSFER SYSTEM OPTIONS FOR NONPUMPABLE WASTES}

Several concepts for retrieval of the nonpumpable residual material present in the GAAT W-9 have been considered. Nonpumpable wastes will be retrieved from W-9 only if risks associated with leaving the material in the tank exceed the allowable guidelines. The options considered for retrieval of the non-pumpable wastes include the following:

1. Retrieval and Packaging System (RPS)

2. Retrieval and Reduction System (RRS)

3. Residual Liquid and Solids Consolidation System (RLSCS)

4. Debris Consolidation System (DCS)

The primary advantages and disadvantages of these options are described in Table 8 . Sections 3.4.1 - 3.4.4 provide brief descriptions of each of the concepts considered for the W-9 nonpumpable waste retrieval and transfer operation. The Pacific Northwest National Laboratory (PNNL) Mechanical and Robotic Systems Group developed the illustrated concepts as part of the Tanks Focus Area Residual Waste Retrieval task.

\subsubsection{RPS}

The RPS concept would retrieve primarily nonpumpable solids along with any incidental liquids or slurry and would package the waste in drums and over-packs for cross-site road transport. The process would add a minimal amount of water to the waste inventory. The RPS concept incorporates the following components:

1) An end effector (Fig 14), which is adapted from a commercially available, hand-held floor-cleaning tool to dislodge and size-reduce the solid waste. The end effector is based on the Aurand MP6 floor-cleaning tool (Fig. 15), which uses a set of rotating, toothed drums to grind the material off the surface being cleaned. A custom suction shroud around the cutter head captures the product. The unit is powered by compressed air, and is fitted with a multiple $\mathrm{X}$-handle fixture for engagement by the gripper end effectors.

2) A collector-segregator system (Fig. 16), consisting of a heavy base plate, an air-powered venturi eductor vacuum head with a suction hose to the end effector and a consumable drum with a screened drain port. The base plate can be locked in place to the top of the riser interface with three screw dogs operated manually from outside the deployment 
chamber, forming an unsealed closure between the tank and the deployment compartment. The base plate has three latches to secure it to the drum.

3) A deployment and drum-handling system (Fig. 17), including a containment enclosure with a transfer tray and airlock, glove ports and windows, fixtures for storing drum lids and bungs, wash-down spray gun, and some hand and pneumatic tools. The deployment system also includes a hoist with a take-up reel for two lifting cables and two air hoses.

The RPS is operated by first lowering the waste collection drum and vacuum power head into the tank and securing it to the base plate on the floor of the tank. The RPS relies on the MLDUA and the Houdini Vehicle to make the in-tank connections and hold and maneuver the floor-cleaning tool. A vacuum eductor is used to vacuum waste into the collection drum. The end effector is used to abrade the solid waste and/or floor and walls to break-up and remove contaminated materials. Once the collection drum is full, it is hoisted from the tank, the power head is removed, and a new lid is placed on the drum. The waste drum is then placed in an over-pack container and transferred to either interim storage or a transport vehicle.

Table 8. Advantages and disadvantages of the options considered for nonpumpable waste-retrieval for GAAT W-9.

\begin{tabular}{|c|c|c|}
\hline Option & Advantages & Disadvantages \\
\hline 1. RPS & $\begin{array}{l}\text { - Provides packaging of } \\
\text { retrieved wastes } \\
\text { - Minimal dilution of } \\
\text { waste } \\
\text { - Can remove } \\
\text { contaminated concrete } \\
\text { and hard materials }\end{array}$ & 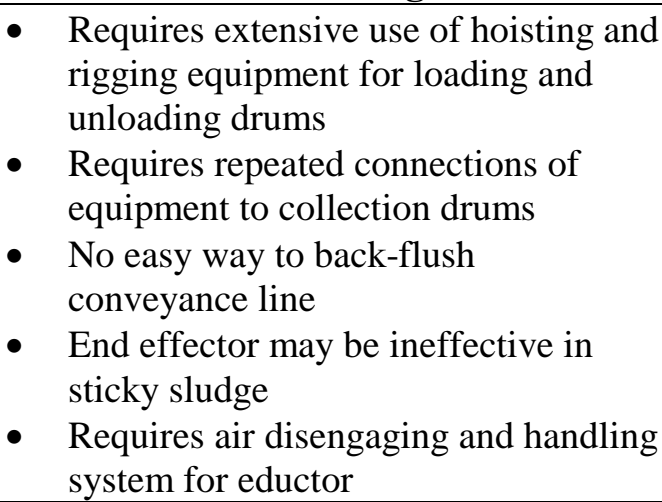 \\
\hline 2. RRS & $\begin{array}{ll}\text { - } & \text { Size reduction of } \\
\text { waste solids } \\
\text { - } \\
\text { Minimal dilution of } \\
\text { waste }\end{array}$ & $\begin{array}{l}\text { - Large and expensive equipment } \\
\text { - Will require development and cold } \\
\text { - } \quad \text { Pesting } \\
\text { - } \quad \text { Requires large air flow rate }\end{array}$ \\
\hline 3. RLSCS & $\begin{array}{ll} & \text { Improved waste- } \\
\text { retrieval efficiency at } \\
\text { low waste levels }\end{array}$ & $\begin{array}{l}\text { Will not retrieve large solids } \\
\text { - Decontamination of inside of waste } \\
\text { drum will be difficult } \\
\text { Effectiveness depends on the ability } \\
\text { of the Houdini Vehicle and operators }\end{array}$ \\
\hline 4. DCS & $\begin{array}{l}\text { Retrieve debris with } \\
\text { minimal dilution }\end{array}$ & $\begin{array}{l}\text { - } \\
\text { Extensive use of Houdini Vehicle } \\
\text { rigging equipment for loading and } \\
\text { unloading waste containers } \\
\text { - Requires that the majority of the } \\
\text { pumpable waste be removed from the } \\
\text { tank }\end{array}$ \\
\hline
\end{tabular}


ORNL Dwg-2000-5842

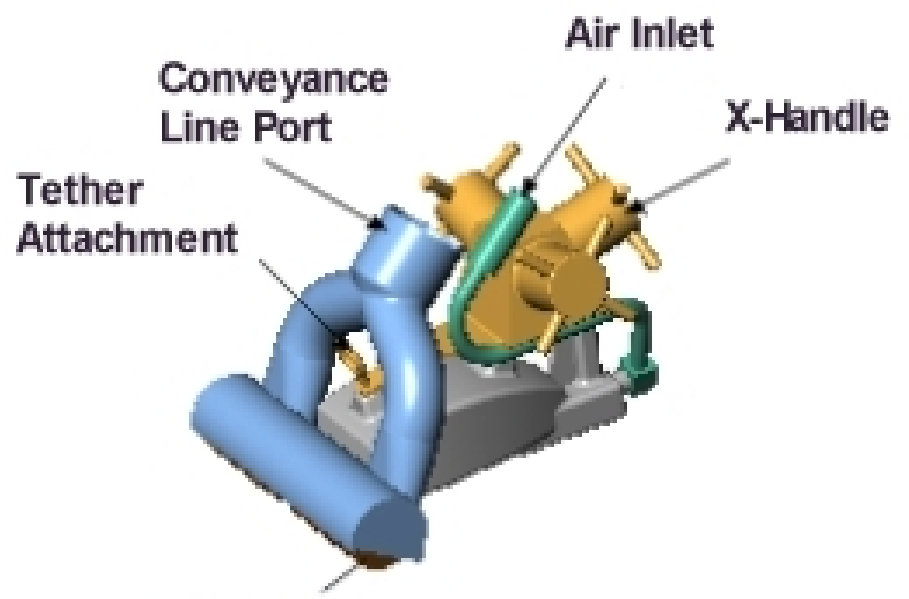

\section{Cutterhead}

Fig. 14. Floor-cleaning end-effector by PNNL.

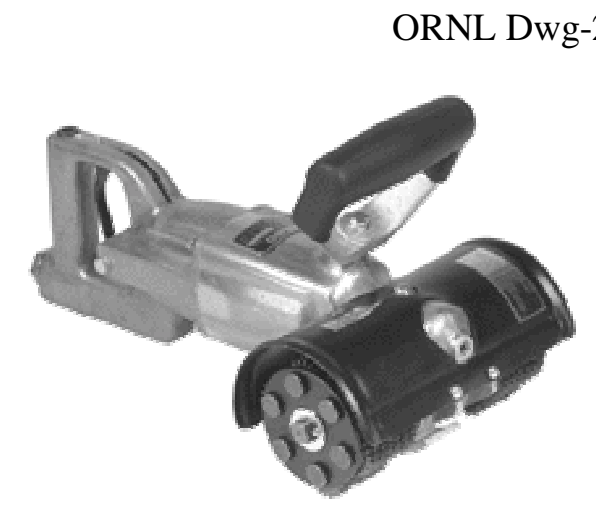

Fig. 15. Aurand MP6 floor-cleaning tool by PNNL. 


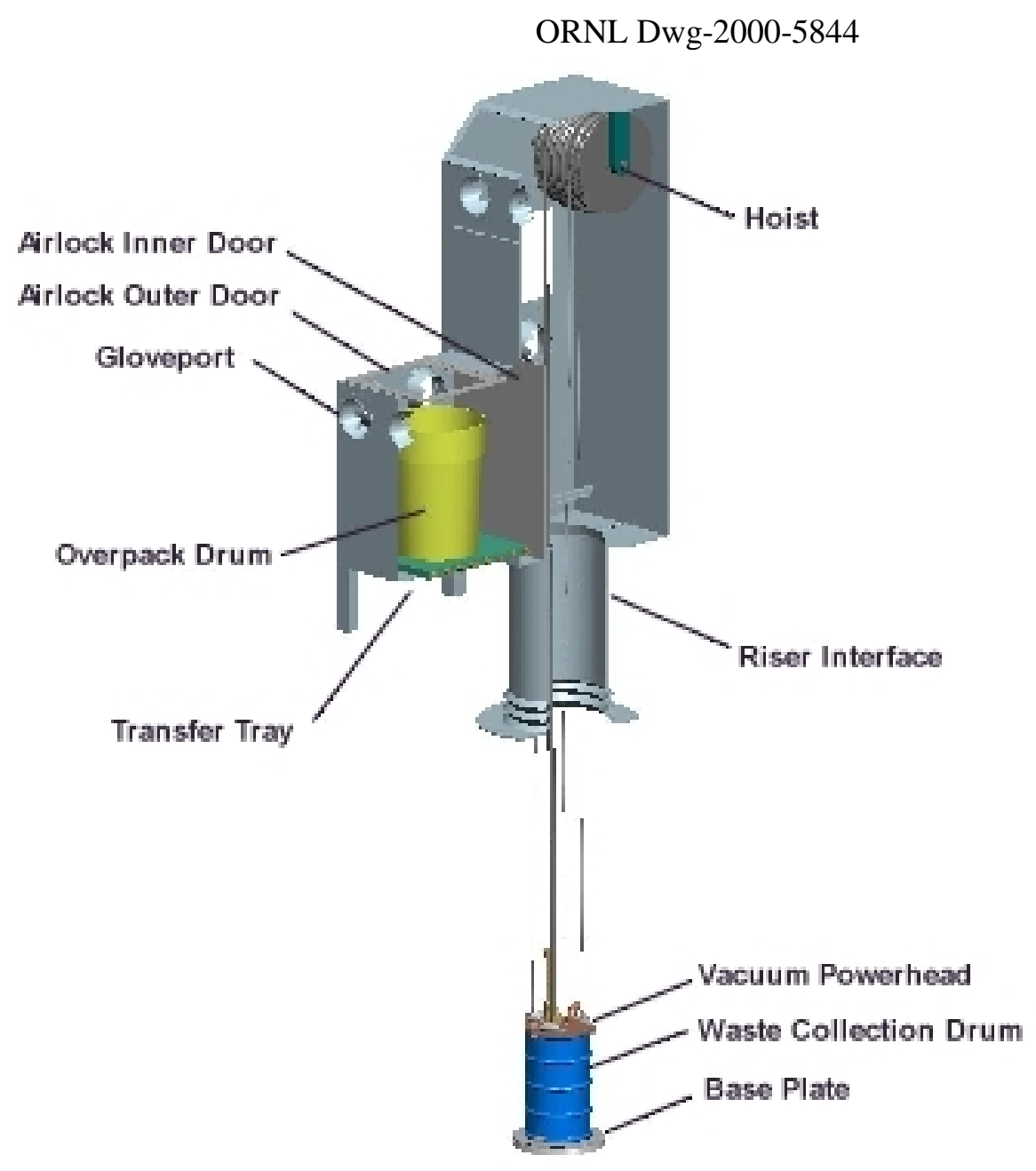

Fig. 16. Collector-segregator system by PNNL. 
ORNL Dwg-2000-5845

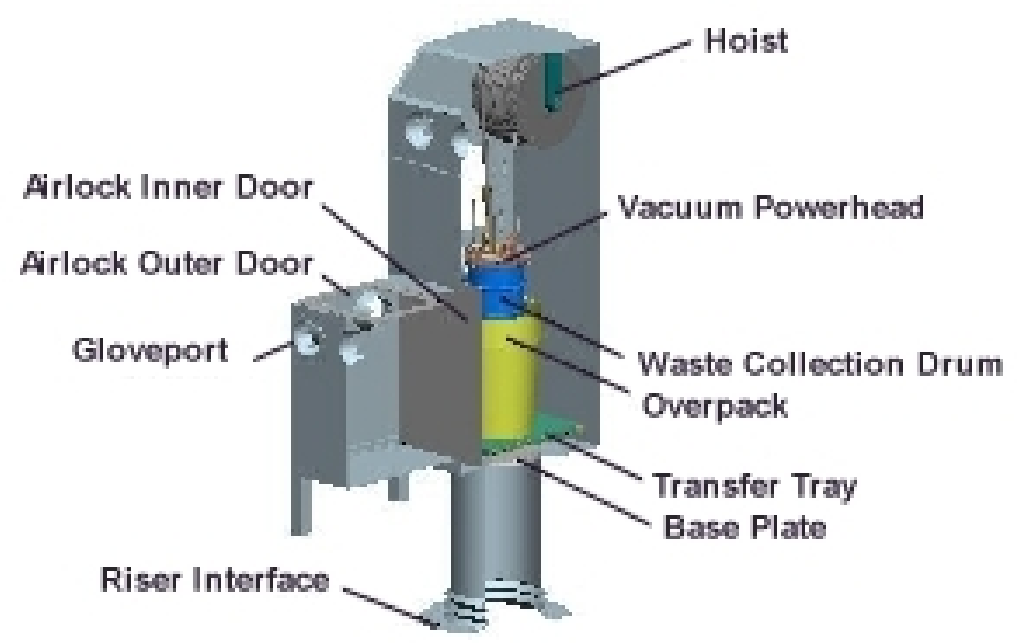

Fig. 17. Deployment and drum-handling system by PNNL.

\subsubsection{RRS}

The objective for the RRS is to retrieve primarily nonpumpable solids along with any incidental liquids or slurry, reduce the solids to pumpable scale, and transfer all the retrieved waste to a separate transfer system for the transfer to the BVESTs. The process will add a minimal amount of water to the waste inventory. The RRS concept, as shown in Fig. 18, incorporates the following major components:

1. A high-powered (200+-scfm, $15-\mathrm{ft}_{2} \mathrm{O}$ static lift) vacuum conveyance system with a cyclone separator. The cyclone separator drains through a remotely operated valve to a horizontal axis ball mill.

2. A horizontal-axis ball mill is used to grind any solids down to an acceptable particle size. The ball mill is a rotating drum, which is lined with abrasion-resistant materials and charged with a quantity of abrasive ceramic balls. An Archimedes screw, which is inside the drum, feeds the contents over a screen section through which finished product is discharged.

3. A capacitance tank and transfer pump receive the ground waste slurry and pump them to the receiving system.

4. A containment sleeve is placed over the tank riser for contamination control and personnel protection.

The RRS is fed by coupling it to either the RPS end effector (Sect. 3.4.1), the CSEE, or to a simple vacuum nozzle, as appropriate for the residue characteristics. In either case the MLDUA and/or Houdini Vehicle is/are needed to place the suction line inlet in the vicinity of the waste. Deployment of the system would be accomplished using an overhead crane. The apparatus could be supported in the tank by a tether, which could attach to a bridge structure to free the crane for other work. A containment sleeve over the tank riser would be used to contain the system. Supporting utilities would include a hydraulic power unit of about $25 \mathrm{hp}$ (if hydraulic motors are used) or three-phase electrical supply, a process water connection for back-flush and mixing jets, and a waste-conveyance line to the receiving system. 


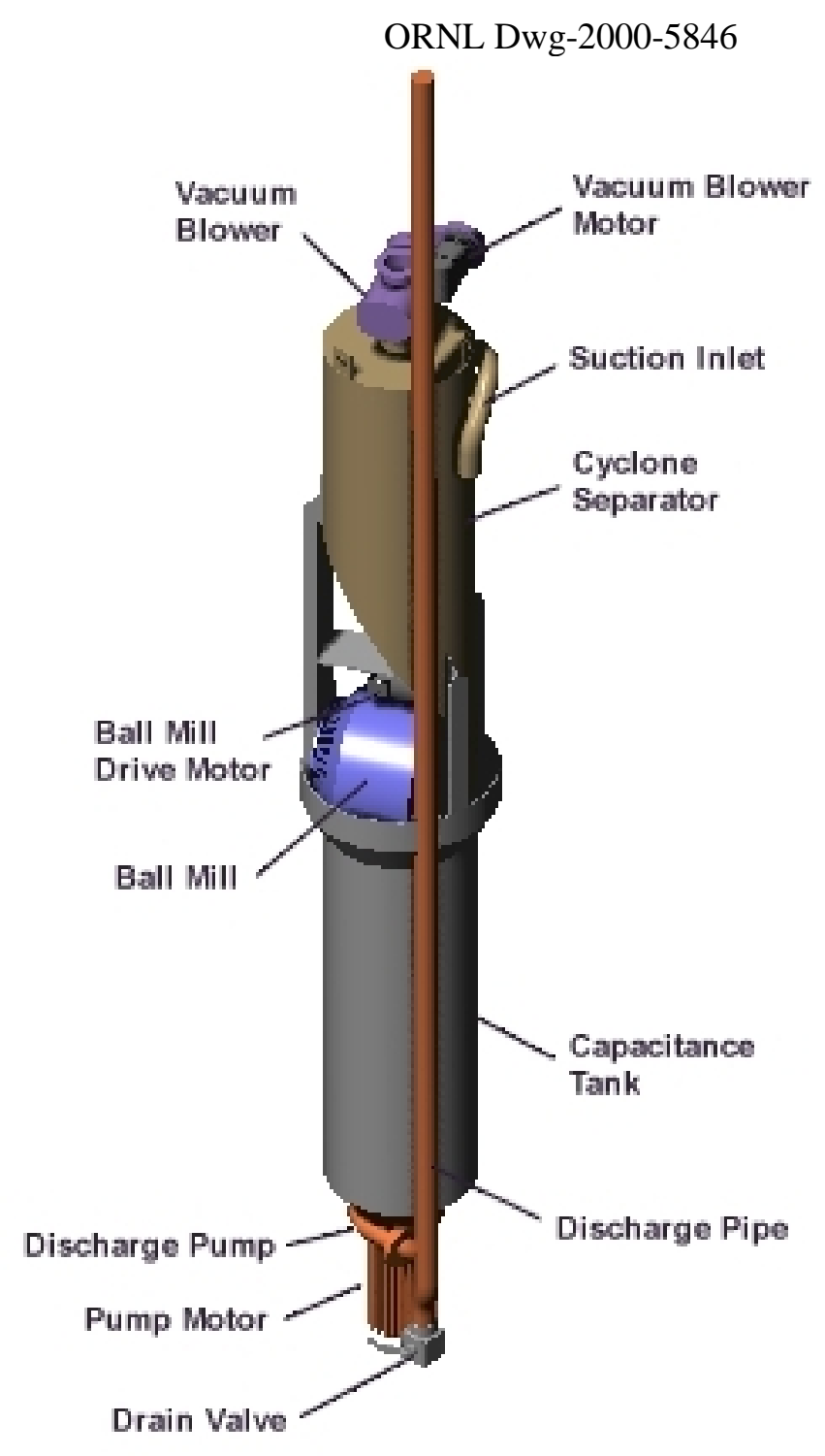

Fig. 18. RRS by PNNL.

\subsubsection{RLSCS}

An RLSCS, which consists of a vacuum cleaner-hopper assembly has been proposed for use in conjunction with the Houdini Vehicle to remove the residual waste from GAAT W-9. The RLSCS was conceived as a solution to the problem of inefficient pumping of the last few inches of residue and liquids off the tank floor with the present WD\&CS. The RLSCS (Fig. 19) includes the following major components:

1. A commercially available, air-powered wet/dry vacuum cleaner, which is powered with a venturi eductor, operating on compressed air (70 scfm at $90 \mathrm{psi}$ and generating up to 110 in.- $\mathrm{H}_{2} \mathrm{O}$ of static lift). The vacuum eductor discharges to the tank atmosphere. The vacuum cleaner is equipped with a wet-dry kit, consisting of a drum extender, a float 
valve, and a wet filter. In this application a stock vacuum cleaner is modified, in which, the stock suction inlet is closed off, and a fixed suction inlet built into the bottom of the canister. The inlet is equipped with a long, grated inlet, which is positioned close to the tank floor, and ported via an internal pipe to a discharge inside and tangential to the head of the drum. A poppet-type dump valve is added in the drum floor. The valve is actuated by a pneumatic cylinder, which is mounted externally on top of the power head using an extension rod through the drum. The external cover over the eductor would be removed to facilitate decontamination.

2. The vacuum unit is mounted in a light framework or harness, which is suspended from a chain hoist.

3. Below the vacuum unit there is a hopper, into which the vacuum drum contents are periodically discharged by opening the dump valve. The hopper is sized to accept the CSEE, allowing the present conveyance system to be operated with a flooded suction inlet. Operation of the CSEE in a flooded state significantly improves the performance of the system. The hopper is hinged to the bottom of the harness and pivots to a vertical orientation for insertion through the tank riser.

4. Two "wings" are hinged to the pivot end of the hopper. When the system is deployed to the tank floor, the hopper is pivoted to the horizontal position, and the wings are spread to form an embayment, with the suction inlet at the head of the bay. Double-acting pneumatic cylinders actuate the hopper pivot and wing extension.

Motive air for the eductor is supplied via a hose on a spring-loaded reel in the deployment housing. Instrument air and a control cable to the solenoid air valves for the cylinders (mounted on the harness) are on a second dual reel. For economy, the electrical control cable is unplugged at the reel hub during hoist operations and plugged in only when evolutions of the hopper and wings are required.

The system is designed for insertion through any free riser with at least a 21-in-diam. opening and a decontamination spray ring. It is unfolded to the deployed configuration and lowered to the tank floor, where it will be rotated to an orientation that is most advantageous to operations with the Houdini Vehicle and the chain hoist. The Houdini Vehicle is used to bulldoze and squeegee material from the tank floor into the "bay" between the "wings" on the in-tank vacuum canister. The vacuum system will then be used to transfer the collected material into the canister through a grated inlet to exclude oversized particles. The contents of the canister will be intermittently dumped into the attached transfer hopper via a dump valve on the bottom of the canister. When both the canister and hopper are full, the CSEE is positioned in the transfer hopper and used to pump out the waste using the existing WD\&CS and a separate transfer system to transfer the waste to the BVESTs.

On completion of the residual-waste-retrieval operation, the CSEE can be used to spray water at the suction inlet of the in-tank vacuum unit while the vacuum is operating. This allows the washing of the inside of the canister. The wash water is dumped to the transfer hopper and transferred out of the tank with the CSEE.

The RLSCS is limited to picking up material, which can pass through the grated inlet and drain to the hopper. Decontamination of the interior of the drum would be difficult; however, an internal sprayer head could be furnished and manually connected to a supply hose upon retraction of the unit. The effectiveness of this system depends on the ability of the Houdini Vehicle and operators to corral the waste in the inlet bay. This may not be a fast or easy operation, and the need for quick operation of the Houdini Vehicle to corral liquids may increase the risk of errors. 


\subsubsection{DCS}

The DCS uses the existing Houdini Vehicle to retrieve debris (i.e., hard solid or other materials and nonpumpable wastes) from the floor of GAAT W-9. The Shilling ${ }^{\mathrm{TM}}$ arm on the Houdini Vehicle can be equipped with a scoop, gripper, or other tooling to facilitate pickup and transport of debris to a small waste container, which is placed on the floor of the tank. These operations would be accomplished after all or most of the pumpable wastes have been removed from the tank. The major equipment components for this concept would include the following:

1. Houdini Vehicle, which is equipped with the appropriate grippers, scoops, and other tooling for grasping and transporting debris.

2. Small-volume waste containers, 5 to 20 gal, to be placed on floor of the tank as needed to contain the debris.

3. Containment structure for decontamination and retrieval of waste containers. The containment enclosure would include a hoist for lowering and retrieving the waste canisters. The containment structure would also include the ability to over-pack the waste containers for subsequent storage, processing, or disposal.

4. A decontamination spray ring would be used to decontaminate the exterior of the waste containers as they are removed from the tank to the containment structure.

A packaging and containment system (similar to that described in Sect. 3.4.1) is envisioned for this option. Waste canisters could be temporarily stored inside W-9 and retrieved at the end of the debris collection operation. The Houdini Vehicle could be used to move the waste canister to and from the vicinity of the withdrawal station. Successful operation and use of the Houdini Vehicle are crucial to the success of this option. 
ORNL Dwg-2000-5847

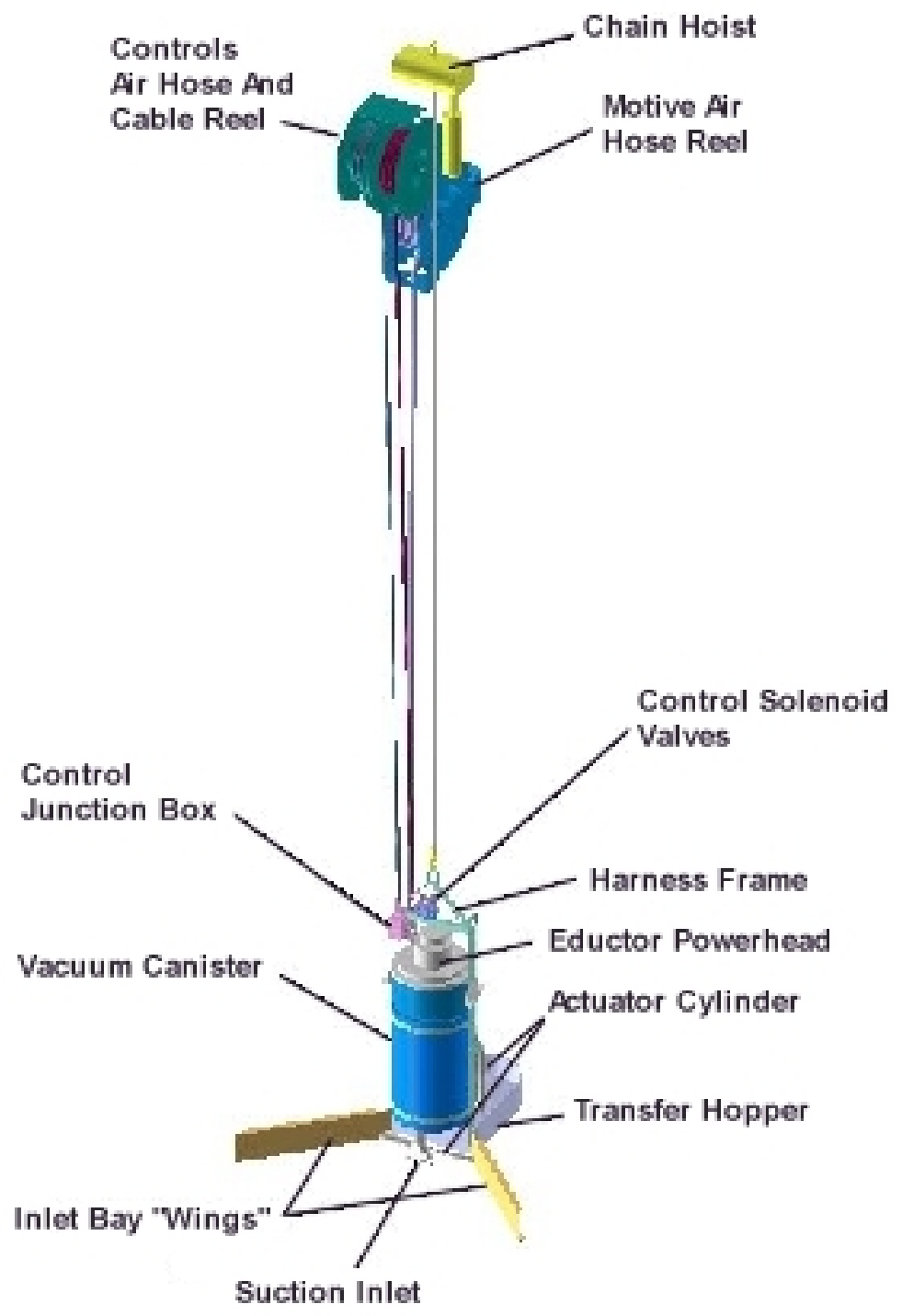

Fig. 19. RSLSC by PNNL. 


\section{RECOMMENDATIONS}

A review of the sample analysis data from the GAAT W-9 sludge samples indicates a minimal risk of violating the WAC for waste transfer to the MVSTs by eliminating the use of a grinder with the sludge transfer. However, it is recommended that a solids classification system, such as the one present on the SCS, be available to ensure that large-diameter particles are prevented from being transferred from W-9 without further conditioning. Samples of the slurry should be periodically taken and analyzed for solids content and particle size to reassess the need for solids classification and/or conditioning.

Our review of the proposed options and alternatives for the W-9 waste-retrieval and -transfer operation indicate a preference for the use of proven systems. Most of the remote systems used in previous waste-retrieval operations are functional and are anticipated to continue to be operational. These systems have proven themselves during previous waste retrieval operations at the GAATs. The operators are familiar with their use and have confidence with their capabilities. Therefore, it is recommended that the existing waste retrieval systems continue to be used for the retrieval of the waste from GAAT W-9. Due to concerns over the continued reliability of the DiscFlo pump, which is currently used to transfer the pumpable waste from W-9 to the MVSTs, and to other considerations, it is recommended that a small booster pump be used to transfer the waste from W-9 to the BVESTs.

Selecting a system for retrieving nonpumpable waste from W-9 cannot be made at this time. The amount of nonpumpable waste that must be removed from W-9 cannot be determined until all the pumpable waste has been removed and the risk of leaving the nonpumpable waste in W-9 can be assessed. It is recommended that the existing waste-retrieval equipment be used to the maximum extent practicable and that the decision on a system for retrieval of the nonpumpable waste be deferred until after the pumpable wastes are removed from the tank.

\section{CONCLUSIONS}

After reviewing the proposed options and alternatives, the decision was made to use the majority of the existing TWRS to transfer the pumpable waste from GAAT W-9 to the BVESTs. A small booster pump will be used in place of the DiscFlo pump to improve the overall reliability of the transfer operations and provide additional access to W-9 for the TWRS. A small accumulator tank will be used along with portions of the SCS to monitor and classify the waste retrieved from W-9 before the waste is transferred to the BVESTs. The AEAT Pulsed Jet Fluidic Mixing system and the existing Moyno transfer pumps will be used to mobilize and transfer the waste from BVEST W-23 to the MVSTs. This option maximizes the use of proven waste retrieval systems and requires only the fabrication of a small surge tank, which can be located in one of the risers in tank W-9. The new components associated with this approach are shown installed at the TTCTF in Fig. 20. This collection of components is referred to as the Waste-Retrieval and -Transfer System (WaRTS). A flow diagram for the entire residual waste-retrieval system for GAAT W-9 is shown in Fig. 21. 


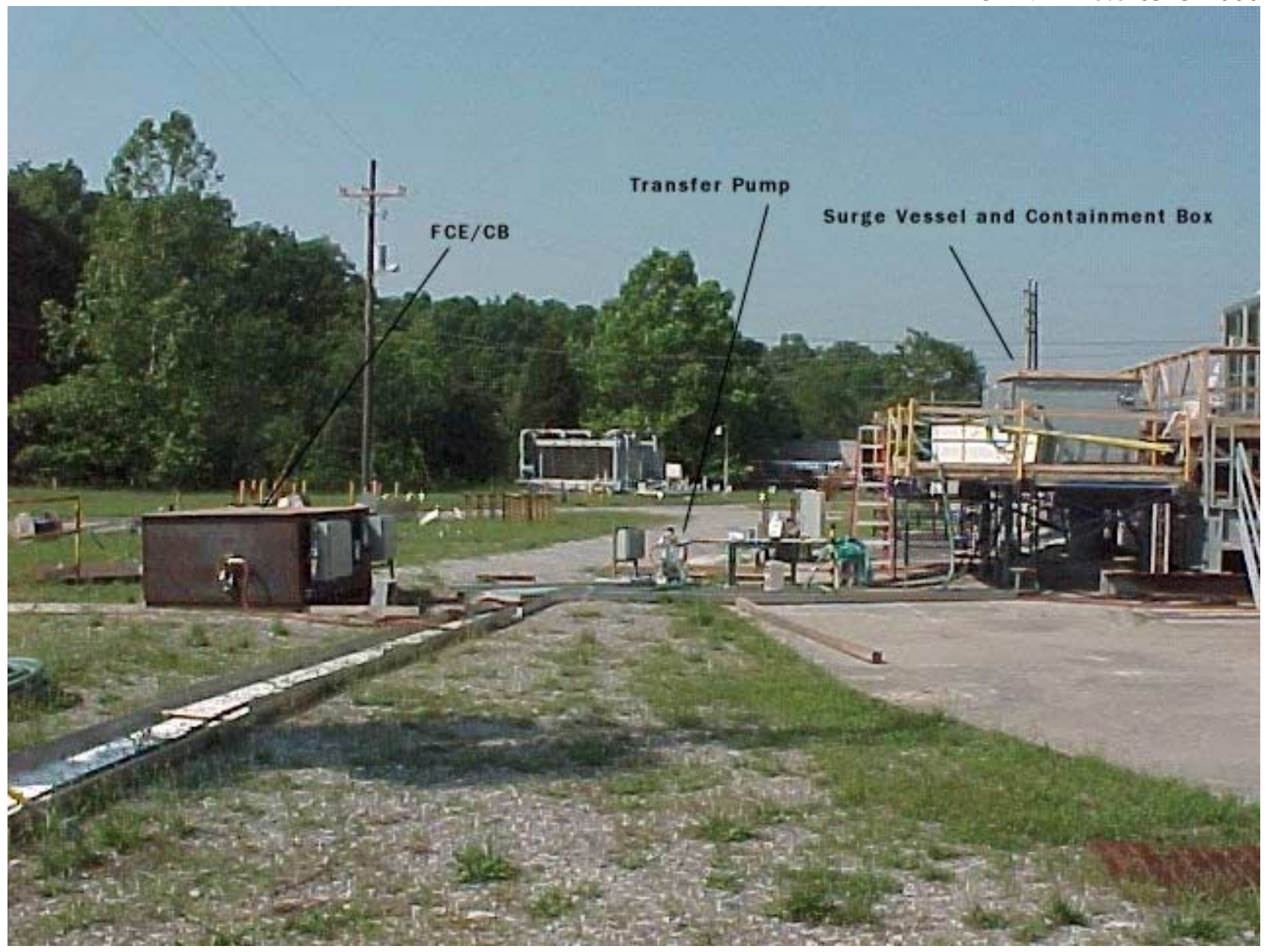

Fig. 20. WaRTS for GAAT W-9 at the ORNL TTCTF. 


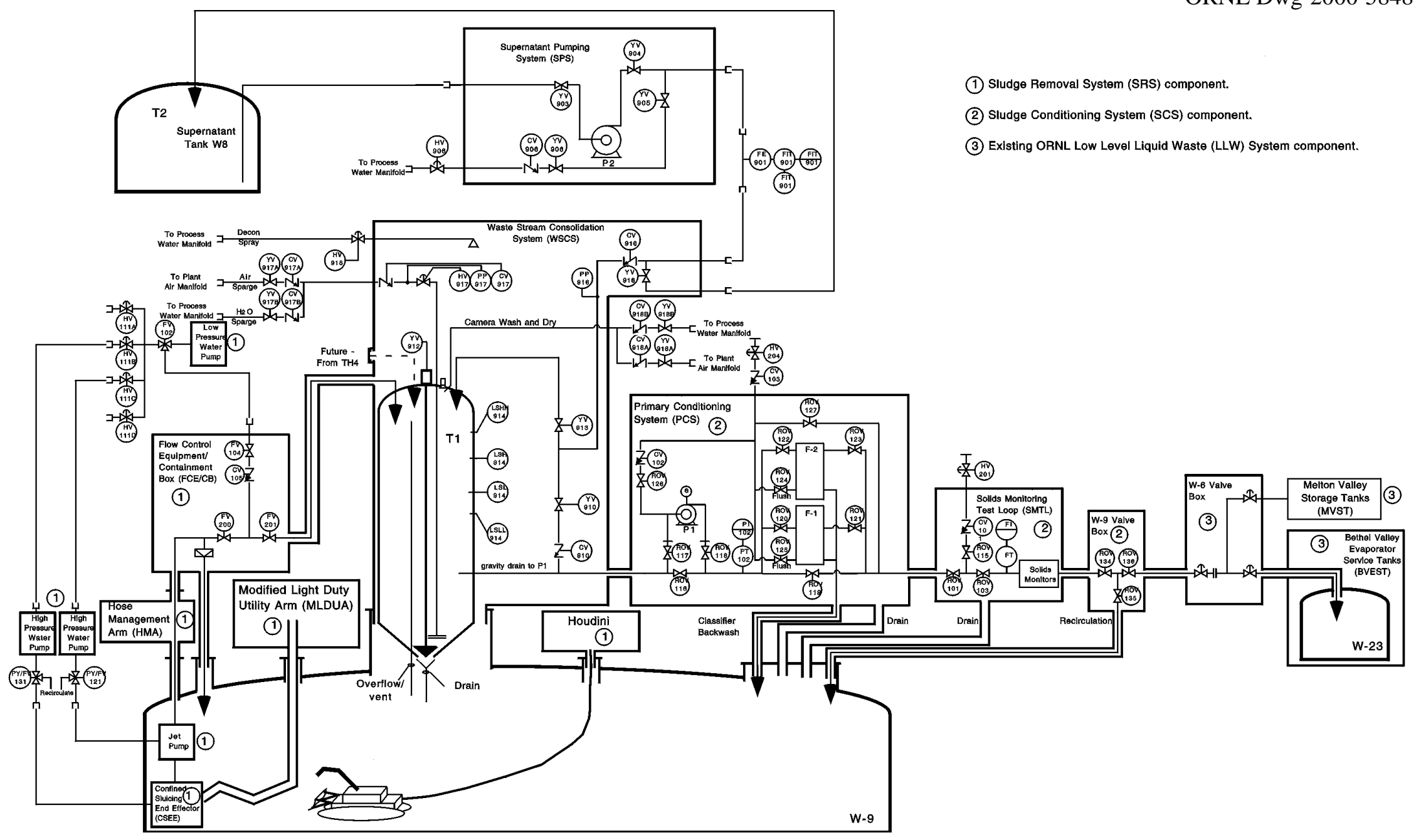

Fig. 21. Flow diagram for the residual waste-retrieval system for GAAT W-9. 


\section{REFERENCES}

1. Weeren, H. O., Sluicing Operations at Gunite Waste Storage Tanks, ORNL/NFW-84/42, Martin Marietta Energy Systems, Inc., Oak Ridge National Laboratory, Oak Ridge, Tennessee, September 1984.

2. Bechtel National, Inc., Results of Fall 1994 Sampling of Gunite and Associated Tanks At the Oak Ridge National Laboratory, Oak Ridge, Tennessee, ORNL/ER/Sub/87-99053/74, Lockheed Martin Energy Systems, Inc., Oak Ridge National Laboratory, Oak Ridge, Tennessee, June 1995.

3. Bechtel National, Inc., Results of 1995 Characterization of Gunite and Associated Tanks at Oak Ridge National Laboratory, Oak Ridge, Tennessee, ORNL/ER/Sub/87-99053/79, Lockheed Martin Energy Systems, Inc., Oak Ridge National Laboratory, Oak Ridge, Tennessee February 1996.

4. Giaquinto, J. M., J. M. Keller, and T. P. Mills, Leaching Studies and Miscellaneous Data for the Gunite and Associated Tanks at Oak Ridge National Laboratory, Oak Ridge, Tennessee, ORNL/ER-364, Lockheed Martin Energy Systems, Inc., Oak Ridge National Laboratory, Oak Ridge National Laboratory, Oak Ridge, Tennessee, March 1996.

5. MACTEC, Evaluation of Phase I and Phase II Sampling and Analysis Data for the Gunite and Associated Tanks at Oak Ridge National Laboratory, Oak Ridge, Tennessee, ORNL/ER-365, Lockheed Martin Energy Systems, Inc., Oak Ridge National Laboratory, Oak Ridge, Tennessee, March 1996.

6. Kent, T. E., S. A. Taylor, J. W. Moore, J. L. Stellern, and K. M. Billingsley, Demonstration of Fluidic Pulse Jet Mixing for a Horizontal Waste Storage Tank, ORNL/TM-13578, Lockheed Martin Energy Research Corp., Oak Ridge National Laboratory, Oak Ridge, Tennessee, January 1998. 
ORNL/TM-2000/251

\section{INTERNAL DISTRIBUTION}

1. M. E. Baldwin

2. J. N. Herndon-Record Copy

3. S. M. Killough

2. B. E. Lewis

3. R. F. Lind

4. P. D. Lloyd

5. ORNL Central Research Library

6. ORNL Laboratory Records

7. K. E. Plummer

8. J. D. Randolph

9. D. E. Rice

10. S. D. Van Hoesen

\section{EXTERNAL DISTRIBUTION}

11. D. H. Bolling, Bechtel Jacobs Company, P. O. Box 2008, MS 6402, Oak Ridge, TN 37831.

12. D. D. Green, U.S. Department of Energy, Oak Ridge Operations Office, P. O. Box 2009, Oak Ridge, TN 37831

13. M. A. Johnson, Tetra-Tech, Inc., Oak Ridge National Laboratory, Oak Ridge, TN 37831.

14. O. D. Mullen, Pacific Northwest National Laboratory, Richland, WA 99352.

15. J. R. Noble-Dial, U.S. Department of Energy, Oak Ridge Operations Office, P. O. Box 2009, Oak Ridge, TN 37831

16. Office of Scientific and Technical Information, DOE Oak Ridge Field Office, P. O. Box 62, Oak Ridge, TN 37831

17. T. P. Pietrok, U.S. Department of Energy, Richland Operations Office, P. O. Box 550, MS K8-50, Richland, WA 99352

18. B. J. Williams, Pacific Northwest National Laboratory, P. O. Box 999, MSIN K9-69, Richland, WA 99352 


\section{Appendix 1. ANALYSIS PLAN FOR GAAT W-9 CORE SAMPLES}

1. Photograph each of the sample tubes.

2. Video selected activities, such as emptying the sample from the collection tube.

3. Empty each sample tube individually into a separate container. Observe the qualitative parameters such as flow ability, adhesiveness, variations in color or texture, etc.

Note: If material is near the bottom of the sample that is taken from the floor of the tank (i.e. Sample No. 3) will not flow from the tube and/or appears to hold it's shape as a solid, it should be separated and handled as a fourth sample.

4. Analyze for percentage of solids [TSS, total solids (TS)], density, radioactive components, and shear strength (if possible) using a representative sample of the sludge from each sample tube.

5. Combine the samples and filter and/or segregate any 'large' solids ( $200 \mu \mathrm{m}$ and larger).

6. For the nonflowing or filtered composite, provide qualitative descriptions of the material (appearance, texture, strength, etc.). Weigh the filtered solids and attempt to dissolve them in nitric acid. Perform a radioactive component analysis of the dissolved material and the undissolved residue, if any. Note the qualitative description and weight of the undissolved material.

7. For the flowing or filtrate material, analyze the composite sample for radioactive constituents, density, solids (TS and TSS), and shear strength.

8. Analyze the composition of the acid dissolution residue and perform an isotopic analysis of the residue. 
Appendix 2. SAMPLE ANALYSIS DATA SUMMARY FOR ALL GAAT W-9 SLUDGE SAMPLES

\begin{tabular}{|c|c|c|c|c|c|c|c|}
\hline \multicolumn{4}{|c|}{ Sample } & \multirow[b]{2}{*}{$\begin{array}{l}\text { PulsAir and Flygt } \\
\text { mixer status }\end{array}$} & \multirow[b]{2}{*}{$\begin{array}{c}\text { SCS } \\
\text { status }\end{array}$} & \multirow[b]{2}{*}{$\begin{array}{c}\text { Analysis } \\
\text { requested }^{b}\end{array}$} & \multirow[b]{2}{*}{$\begin{array}{c}\text { Data } \\
\text { summary }^{c}\end{array}$} \\
\hline $\begin{array}{l}\text { Collection } \\
\text { date }\end{array}$ & No. & Type & Location $^{a}$ & & & & \\
\hline $11-17-98$ & $\begin{array}{l}\text { GAAT- } \\
\text { W9-012 }\end{array}$ & Grab & ND & ND & $\begin{array}{l}\text { System not } \\
\text { online }\end{array}$ & $\begin{array}{l}\text { Density, percent } \\
\text { solids; G-alpha, } \\
\text { beta, and gamma }\end{array}$ & ND \\
\hline $12-10-98$ & $\begin{array}{l}\text { GAAT- } \\
\text { W9-013 }\end{array}$ & Grab & ND & ND & $\begin{array}{l}\text { System not } \\
\text { online }\end{array}$ & $\begin{array}{l}\text { Density, total } \\
\text { solids, suspended } \\
\text { solids; G-alpha, } \\
\text { beta, and gamma }\end{array}$ & ND \\
\hline $12-10-98$ & $\begin{array}{l}\text { GAAT- } \\
\text { W9-014 }\end{array}$ & Grab & ND & ND & $\begin{array}{l}\text { System not } \\
\text { online }\end{array}$ & $\begin{array}{l}\text { Density, total } \\
\text { solids, suspended } \\
\text { solids; G-alpha, } \\
\text { beta, and gamma }\end{array}$ & ND \\
\hline $1-20-99$ & $\begin{array}{l}\text { GAAT- } \\
\text { W9-015 }\end{array}$ & Grab & $\begin{array}{l}\text { Isolok }^{\mathrm{TM}} \\
\text { sampler } \\
\text { Tank volume = } \\
119,000 \mathrm{gal}\end{array}$ & ND & $\begin{array}{l}\text { Normal } \\
\text { system } \\
\text { operation }\end{array}$ & $\begin{array}{l}\text { Density, total } \\
\text { solids, suspended } \\
\text { solids; dissolved } \\
\text { solids, G-beta and } \\
\text { gamma }\end{array}$ & $\begin{array}{l}{ }^{60} \mathrm{Co}=6.8 \mathrm{E}+0 \\
{ }^{137} \mathrm{Cs}=8.4 \mathrm{E}+3 \\
\text { G-beta }=9.8 \mathrm{E}+3 \\
\text { Density }=0.9890 \\
\text { pH }=10.2 \\
\mathrm{TDS}=18,700 \\
\mathrm{TS}=19,600 \\
\mathrm{TSS}=<100\end{array}$ \\
\hline $2-3-99$ & $\begin{array}{l}\text { GAAT- } \\
\text { W9-016 }\end{array}$ & Grab & $\begin{array}{l}\text { NE riser, } 17-\mathrm{ft} \\
\text { depth } \\
\text { Tank volume = } \\
\sim 118,898 \text { gal }\end{array}$ & $\begin{array}{l}\text { Supply Tank at } \sim 35 \text { psig } \\
\text { Dwell time } 10 \mathrm{~s} \\
\text { Injection time, } 1 \mathrm{~s} \\
\text { Total run time } \sim 8 \mathrm{~h} \\
\text { prior to sample } \\
\text { collection }\end{array}$ & $\begin{array}{l}\text { System not } \\
\text { online }\end{array}$ & $\begin{array}{l}{ }^{60} \mathrm{Co},{ }^{137} \mathrm{Cs}, \mathrm{G}- \\
\text { beta, density, } \mathrm{pH} \text {, } \\
\mathrm{TDS}, \mathrm{TS} \text {, TSS }\end{array}$ & $\begin{array}{l}{ }^{60} \mathrm{Co}=1.0 \mathrm{E}+2 \\
{ }^{137} \mathrm{Cs}=1.7 \mathrm{E}+5 \\
\mathrm{G}-\text { beta }=3.1 \mathrm{E}+5 \\
\text { Density }=1.04 \\
\mathrm{pH}=10.10 \\
\mathrm{TDS}=21,800 \\
\mathrm{TS}=47,200 \\
\mathrm{TSS}=25,400\end{array}$ \\
\hline
\end{tabular}


SAMPLE ANALYSIS DATA SUMMARY FOR ALL GAAT W-9 SLUDGE SAMPLES (Continued)

\begin{tabular}{|c|c|c|c|c|c|c|c|}
\hline \multicolumn{4}{|c|}{ Sample } & \multirow[b]{2}{*}{$\begin{array}{l}\text { PulsAir and Flygt } \\
\text { mixer status }\end{array}$} & \multirow[b]{2}{*}{$\begin{array}{c}\text { SCS } \\
\text { status }\end{array}$} & \multirow[b]{2}{*}{$\begin{array}{c}\text { Analysis } \\
\text { requested }^{b}\end{array}$} & \multirow[b]{2}{*}{$\begin{array}{c}\text { Data } \\
\text { summary }^{c}\end{array}$} \\
\hline $\begin{array}{c}\text { Collection } \\
\text { date }\end{array}$ & No. & Type & Location $^{a}$ & & & & \\
\hline $2-3-99$ & $\begin{array}{l}\text { GAAT- } \\
\text { W9-017 }\end{array}$ & Grab & $\begin{array}{l}\text { NE Riser, } 20-\mathrm{ft} \\
\text { depth } \\
\text { Tank volume = } \\
\sim 118,898 \mathrm{gal}\end{array}$ & $\begin{array}{l}\text { Supply Tank @ 35 } \\
\text { psig } \\
\text { Dwell time } 10 \mathrm{~s} \\
\text { Injection time } 1 \mathrm{~s} \\
\text { Total run time, } \sim 8 \mathrm{~h} \\
\text { prior to sample } \\
\text { collection }\end{array}$ & $\begin{array}{l}\text { System not } \\
\text { online }\end{array}$ & $\begin{array}{l}{ }^{60} \mathrm{Co},{ }^{137} \mathrm{Cs}, \mathrm{G}- \\
\text { beta, density, } \mathrm{pH} \text {, } \\
\text { TDS, TS, TSS }\end{array}$ & $\begin{array}{l}{ }^{60} \mathrm{Co}=1.0 \mathrm{E}+2 \\
{ }^{137} \mathrm{Cs}=2.6 \mathrm{E}+5 \\
\mathrm{G}-\mathrm{beta}=4.8 \mathrm{E}+5 \\
\text { Density }=1.04 \\
\text { pH }=10.11 \\
\text { TDS }=21,300 \\
\mathrm{TS}=60,400 \\
\mathrm{TSS}=39,100\end{array}$ \\
\hline $2-11-99$ & $\begin{array}{l}\text { GAAT- } \\
\text { W9-018 }\end{array}$ & Grab & Isolok sampler & $\begin{array}{l}\text { Supply tank at } \sim 30 \mathrm{psig} \\
\text { Dwell time } 10 \mathrm{~s} \\
\text { Injection time } 1.0 \mathrm{~s} \\
\text { Total run time, } \sim 45 \mathrm{~min} \\
\text { at these settings, } \\
\text { changed injection time } \\
\text { to } 0.5 \mathrm{~s} \text { for } \sim 4 \mathrm{~h} \text { prior } \\
\text { to sample collection }\end{array}$ & $\begin{array}{l}\text { Normal } \\
\text { system } \\
\text { operation }\end{array}$ & $\begin{array}{l}{ }^{60} \mathrm{Co},{ }^{137} \mathrm{Cs}, \mathrm{G}- \\
\text { beta, density, } \mathrm{pH} \text {, } \\
\text { TDS, TS, TSS }\end{array}$ & $\begin{array}{l}{ }^{60} \mathrm{Co}=1.6 \mathrm{E}+2 \\
{ }^{137} \mathrm{Cs}=5.0 \mathrm{E}+5 \\
\mathrm{G}-\mathrm{beta}=7.6 \mathrm{E}+5 \\
\text { Density }=1.06 \\
\text { pH }=\text { ND } \\
\text { TDS = 22,700 } \\
\text { TS }=78,000 \\
\text { TSS }=55,900\end{array}$ \\
\hline $2-16-99$ & $\begin{array}{l}\text { GAAT- } \\
\text { W9-019 }\end{array}$ & Grab & $\begin{array}{l}\text { NE Riser, 18-ft } \\
\text { depth } \\
\text { Tank volume = } \\
\sim 102,000 \text { gal }\end{array}$ & $\begin{array}{l}\text { Supply tank at } \sim 35 \text { psig } \\
\text { Dwell time } 9 \mathrm{~s} \\
\text { Injection time, } 1 \mathrm{~s} \\
\text { Total run time, } 3 \mathrm{~h} 15 \\
\text { min ( } 45 \text { min at these } \\
\text { settings prior to sample } \\
\text { collection, several } \\
\text { adjustments were made } \\
\text { on the system } \\
\text { throughout the shift. } \\
\text { See log entries for } 2- \\
16-99 \text { ) }\end{array}$ & $\begin{array}{l}\text { System not } \\
\text { online }\end{array}$ & $\begin{array}{l}{ }^{60} \mathrm{Co},{ }^{137} \mathrm{Cs}, \mathrm{G}- \\
\text { beta, density, } \mathrm{pH} \text {, } \\
\text { TDS, TS, TSS }\end{array}$ & $\begin{array}{l}{ }^{60} \mathrm{Co}=4.2 \mathrm{E}+1 \\
{ }^{137} \mathrm{Cs}=3.2 \mathrm{E}+5 \\
\text { Total activity }=4.7 \mathrm{E}+5 \\
\text { Density }=1.04 \\
\mathrm{TDS}=23,200 \\
\mathrm{TS}=55,400 \\
\mathrm{TSS}=32,700\end{array}$ \\
\hline
\end{tabular}


SAMPLE ANALYSIS DATA SUMMARY FOR ALL GAAT W-9 SLUDGE SAMPLES (Continued)

\begin{tabular}{|c|c|c|c|c|c|c|c|}
\hline \multicolumn{4}{|c|}{ Sample } & \multirow[b]{2}{*}{$\begin{array}{l}\text { PulsAir and Flygt } \\
\text { mixer status }\end{array}$} & \multirow[b]{2}{*}{$\begin{array}{c}\text { SCS } \\
\text { status }\end{array}$} & \multirow[b]{2}{*}{$\begin{array}{c}\text { Analysis } \\
\text { requested }^{b}\end{array}$} & \multirow[b]{2}{*}{$\begin{array}{c}\text { Data } \\
\text { summary }^{c}\end{array}$} \\
\hline $\begin{array}{c}\text { Collection } \\
\text { date }\end{array}$ & No. & Type & Location $^{a}$ & & & & \\
\hline $2-16-99$ & $\begin{array}{l}\text { GAAT- } \\
\text { W9-020 }\end{array}$ & Grab & $\begin{array}{l}\text { NE Riser, } 20-\mathrm{ft} \\
\text { depth } \\
\text { Tank volume = } \\
\sim 102,000 \mathrm{gal}\end{array}$ & $\begin{array}{l}\text { Supply tank at } \sim 35 \text { psig } \\
\text { Dwell Time } 9 \mathrm{~s} \\
\text { Injection Time, } 1 \mathrm{~s} \\
\text { Total run time, } 3 \mathrm{~h} 15 \\
\text { min ( } 45 \text { min. At these } \\
\text { settings prior to sample } \\
\text { collection, several } \\
\text { adjustments were made } \\
\text { on the system } \\
\text { throughout the shift. } \\
\text { See log entries for } 2- \\
16-99 \text { ) }\end{array}$ & $\begin{array}{l}\text { System not } \\
\text { online }\end{array}$ & $\begin{array}{l}{ }^{60} \mathrm{Co},{ }^{137} \mathrm{Cs}, \mathrm{G}- \\
\text { beta, density, } \mathrm{pH} \text {, } \\
\mathrm{TDS}, \mathrm{TS}, \mathrm{TSS}\end{array}$ & $\begin{array}{l}{ }^{60} \mathrm{Co}=9.4 \mathrm{E}+1 \\
{ }^{137} \mathrm{Cs}=3.3 \mathrm{E}+5 \\
\text { Total activity }=4.8 \mathrm{E}+5 \\
\text { Density }=1.04 \\
\mathrm{TDS}=22,700 \\
\mathrm{TS}=55,600 \\
\mathrm{TSS}=33,300\end{array}$ \\
\hline $2-17-99$ & $\begin{array}{l}\text { GAAT- } \\
\text { W9-021 }\end{array}$ & Grab & Isolok sampler & $\begin{array}{l}\text { Supply tank at } \sim 35 \text { psig } \\
\text { Dwell time, } 9 \mathrm{~s} \\
\text { Injection time, } 1 \mathrm{~s} \\
\text { Total run time, } 3 \mathrm{~h} 5 \\
\text { min }\end{array}$ & $\begin{array}{l}\text { Normal } \\
\text { system } \\
\text { operation }\end{array}$ & $\begin{array}{l}{ }^{60} \mathrm{Co},{ }^{137} \mathrm{Cs}, \mathrm{G}- \\
\text { beta, density, pH, } \\
\text { TDS, TS, TSS, } \\
\text { supernate density, } \\
\text { particle size } \\
\text { distribution }\end{array}$ & ND \\
\hline $2-22-99$ & $\begin{array}{l}\text { GAAT- } \\
\text { W9-022 }\end{array}$ & Grab & Isolok sampler & $\begin{array}{l}\text { Supply tank at } \sim 35 \text { psig } \\
\text { Dwell time, } 18 \mathrm{~s} \\
\text { Injection time, } 1 \mathrm{~s} \\
\text { Total run time, } 2 \text { h } 50 \\
\text { min }\end{array}$ & $\begin{array}{l}\text { Normal } \\
\text { system } \\
\text { operation }\end{array}$ & $\begin{array}{l}{ }^{60} \mathrm{Co},{ }^{137} \mathrm{Cs}, \mathrm{G}- \\
\text { beta, density, pH, } \\
\text { TDS, TS, TSS, } \\
\text { supernate density, } \\
\text { particle size } \\
\text { distribution }\end{array}$ & $\begin{array}{l}{ }^{60} \mathrm{Co}=9.9 \mathrm{E}+1 \\
{ }^{137} \mathrm{Cs}=2.9 \mathrm{E}+5 \\
\mathrm{G}-\mathrm{beta}=4.3 \mathrm{E}+5 \\
\text { Density }=1.04 \\
\text { pH }=\text { ND } \\
\text { TDS = ND } \\
\text { TS }=51,400 \\
\text { TSS }=29,700\end{array}$ \\
\hline $2-25-99$ & $\begin{array}{l}\text { GAAT- } \\
\text { W9-023 }\end{array}$ & Grab & Isolok sampler & $\begin{array}{l}\text { Supply tank at } \sim 35 \text { psig } \\
\text { Dwell time, } 14 \mathrm{~s} \\
\text { Injection time, } 1 \mathrm{~s} \\
\text { Total run time, } 5 \mathrm{~h} 5 \\
\text { min }\end{array}$ & $\begin{array}{l}\text { Normal } \\
\text { system } \\
\text { operation }\end{array}$ & $\begin{array}{l}{ }^{60} \mathrm{Co},{ }^{137} \mathrm{Cs}, \mathrm{G}- \\
\text { beta, density, pH, } \\
\text { TDS, TS, TSS, } \\
\text { supernate density, } \\
\text { particle size } \\
\text { distribution }\end{array}$ & $\begin{array}{l}{ }^{60} \mathrm{Co}=5.8 \mathrm{E}+1 \\
{ }^{137} \mathrm{Cs}=2.7 \mathrm{E}+5 \\
\mathrm{G}-\mathrm{beta}=4.1 \mathrm{E}+5 \\
\text { Density }=1.04 \\
\text { pH }=\text { ND } \\
\text { TDS = ND } \\
\text { TS }=50,900 \\
\text { TSS }=28,600\end{array}$ \\
\hline
\end{tabular}


SAMPLE ANALYSIS DATA SUMMARY FOR ALL GAAT W-9 SLUDGE SAMPLES (Continued)

\begin{tabular}{|c|c|c|c|c|c|c|c|}
\hline \multicolumn{4}{|c|}{ Sample } & \multirow[b]{2}{*}{$\begin{array}{l}\text { PulsAir and Flygt } \\
\text { mixer status }\end{array}$} & \multirow[b]{2}{*}{$\begin{array}{l}\text { SCS } \\
\text { status }\end{array}$} & \multirow[b]{2}{*}{$\begin{array}{c}\text { Analysis } \\
\text { requested }^{b}\end{array}$} & \multirow[b]{2}{*}{$\begin{array}{c}\text { Data } \\
\text { summary }^{c}\end{array}$} \\
\hline $\begin{array}{c}\text { Collection } \\
\text { date }\end{array}$ & No. & Type & Location $^{a}$ & & & & \\
\hline $3-2-99$ & $\begin{array}{l}\text { GAAT- } \\
\text { W9-024 }\end{array}$ & Grab & Isolok sampler & $\begin{array}{l}\text { Supply tank at } \sim 35 \text { psig } \\
\text { Dwell time, } 10 \mathrm{~s} \\
\text { Injection time, } 1 \mathrm{~s} \\
\text { Total run time, } 2 \mathrm{~h} 20 \\
\text { min }\end{array}$ & $\begin{array}{l}\text { Normal } \\
\text { system } \\
\text { operation }\end{array}$ & $\begin{array}{l}{ }^{60} \mathrm{Co},{ }^{137} \mathrm{Cs}, \mathrm{G}- \\
\text { beta, density, pH, } \\
\text { TDS, TS, TSS, } \\
\text { supernate density, } \\
\text { particle size } \\
\text { distribution }\end{array}$ & $\begin{array}{l}{ }^{60} \mathrm{Co}=6.4 \mathrm{E}+1 \\
{ }^{137} \mathrm{Cs}=2.7 \mathrm{E}+5 \\
\mathrm{G}-\mathrm{beta}=3.9 \mathrm{E}+5 \\
\text { Density }=1.035 \\
\text { pH }=\text { ND } \\
\text { TDS }=21,400 \\
\text { TS }=45,200 \\
\text { TSS }=23,400\end{array}$ \\
\hline $3-5-99$ & $\begin{array}{l}\text { GAAT- } \\
\text { W9-025 }\end{array}$ & Grab & Isolok sampler & $\begin{array}{l}\text { Supply Tank@ } ~ 35 p s i g \\
\text { Dwell time, } 14 \mathrm{~s} \\
\text { Injection time, } 1 \mathrm{~s} \\
\text { Total run time, } \sim 3 \mathrm{~h}\end{array}$ & $\begin{array}{l}\text { Normal } \\
\text { system } \\
\text { operation }\end{array}$ & $\begin{array}{l}\text { G-beta gamma, } \\
\text { density, pH, TDS, } \\
\text { TS, TSS, } \\
\text { supernate density, } \\
\text { particle size } \\
\text { distribution }\end{array}$ & $\begin{array}{l}{ }^{60} \mathrm{Co}=1.1 \mathrm{E}+1 \\
{ }^{137} \mathrm{Cs}=2.4 \mathrm{E}+5 \\
\text { Density }=1.034 \\
\text { pH }=\text { ND } \\
\text { TDS }=21,300 \\
\mathrm{TS}=43,300 \\
\mathrm{TSS}=21,900 \\
\text { Total activity }=3.6 \mathrm{E}+5\end{array}$ \\
\hline $3-11-99$ & $\begin{array}{l}\text { GAAT- } \\
\text { W9-026 }\end{array}$ & Grab & Isolok sampler & $\begin{array}{l}\text { Supply tank at } \sim 35 \text { psig } \\
\text { Dwell time, } 18 \mathrm{~s} \\
\text { Injection time, } 1 \mathrm{~s} \\
\text { Total run time, } 2 \mathrm{~h} 20 \\
\text { min }\end{array}$ & $\begin{array}{l}\text { Normal } \\
\text { system } \\
\text { operation }\end{array}$ & $\begin{array}{l}\text { G-beta gamma, } \\
\text { density, pH, TDS, } \\
\text { TS, TSS, } \\
\text { supernate density, } \\
\text { particle size } \\
\text { distribution }\end{array}$ & $\begin{array}{l}{ }^{60} \mathrm{Co}=<160 \\
{ }^{137} \mathrm{Cs}=2.4 \mathrm{E}+5 \\
\text { Density }=1.035 \\
\mathrm{pH}=\mathrm{ND} \\
\mathrm{TDS}=21,300 \\
\mathrm{TS}=43,300 \\
\mathrm{TSS}=22,300 \\
\text { Total activity }=3.4 \mathrm{E}+5\end{array}$ \\
\hline
\end{tabular}


SAMPLE ANALYSIS DATA SUMMARY FOR ALL GAAT W-9 SLUDGE SAMPLES (Continued)

\begin{tabular}{|c|c|c|c|c|c|c|c|}
\hline \multicolumn{4}{|c|}{ Sample } & \multirow[b]{2}{*}{$\begin{array}{l}\text { PulsAir and Flygt } \\
\text { mixer status }\end{array}$} & \multirow[b]{2}{*}{$\begin{array}{c}\text { SCS } \\
\text { status }\end{array}$} & \multirow[b]{2}{*}{$\begin{array}{c}\text { Analysis } \\
\text { requested }^{b}\end{array}$} & \multirow[b]{2}{*}{$\begin{array}{c}\text { Data } \\
\text { summary }^{c}\end{array}$} \\
\hline $\begin{array}{c}\text { Collection } \\
\text { date }\end{array}$ & No. & Type & Location $^{a}$ & & & & \\
\hline $3-25-99$ & $\begin{array}{l}\text { GAAT- } \\
\text { W9-027 }\end{array}$ & Grab & ND & ND & ND & $\begin{array}{l}\text { See GAAT } \\
\text { Sampling and } \\
\text { Analysis Plan }\end{array}$ & $\begin{array}{l}{ }^{238} \mathrm{U}=16 \% \\
{ }^{233} \mathrm{U} /{ }^{234} \mathrm{U}=17.4 \% \\
{ }^{239} \mathrm{Pu} /{ }^{240} \mathrm{Pu}=16.8 \% \\
{ }^{238} \mathrm{Pu} /{ }^{241} \mathrm{Am}=18.8 \% \\
{ }^{244} \mathrm{Cm}=31 \% \\
{ }^{60} \mathrm{Co}=1.5 \mathrm{E}+2 \\
{ }^{137} \mathrm{Cs}=5.4 \mathrm{E}+5 \\
\text { Density }=1.052 \\
\text { G-Alpha }=1.9 \mathrm{E}+3 \\
\text { TS }=6,9000 \\
\text { TSS }=50,900 \\
\text { Total activity }=7.8 \mathrm{E}+5\end{array}$ \\
\hline $4-8-99$ & $\begin{array}{l}\text { GAAT- } \\
\text { W9-028 }\end{array}$ & Grab & NE Riser & ND & ND & $\begin{array}{l}\text { TS, Density, } \\
\text { Isotopic beta- } \\
\text { gamma scan, } \\
\text { gross } \\
\text { radioactivity }\end{array}$ & $\begin{array}{l}{ }^{238} \mathrm{U}=46 \% \\
{ }^{233} \mathrm{U} /{ }^{234} \mathrm{U}=54 \% \\
{ }^{60} \mathrm{Co}=7.2 \mathrm{E}+0 \\
{ }^{137} \mathrm{Cs}=3.6 \mathrm{E}+4 \\
\text { Density }=1.02 \\
\mathrm{G}-\mathrm{Alpha}=5.7 \mathrm{E}+3 \\
\mathrm{TS}=20000 \\
\text { Total activity }=3.7 \mathrm{E}+4\end{array}$ \\
\hline $5-21-99$ & $\begin{array}{l}\text { GAAT- } \\
\text { W9-029 }\end{array}$ & Grab & $\begin{array}{l}\text { NE Riser, 4-ft } \\
\text { below } \\
\text { supernate } \\
\text { level } \\
\text { Tank volume = } \\
\sim 129,627 \mathrm{gal}\end{array}$ & $\begin{array}{l}\text { Supply tank at } \sim 35 \mathrm{psig} \\
\text { Dwell time, } 8 \mathrm{~s} \\
\text { Injection time, } 1.5 \mathrm{~s} \\
\text { Total run time, } 2 \mathrm{~h}\end{array}$ & $\begin{array}{l}\text { Normal } \\
\text { system } \\
\text { operation }\end{array}$ & $\begin{array}{l}\text { G-alpha, G-beta, } \\
\text { beta, gamma, } \\
\text { alpha scan, } \\
\text { density }\end{array}$ & $\begin{array}{l}{ }^{238} \mathrm{U}=14.7 \% \\
{ }^{233} \mathrm{U} /{ }^{234} \mathrm{U}=16.2 \% \\
{ }^{239} \mathrm{Pu} /{ }^{240} \mathrm{Pu}=15.9 \% \\
{ }^{238} \mathrm{Pu} /{ }^{241} \mathrm{Am}=18.5 \% \\
{ }^{244} \mathrm{Cm}=34.8 \% \\
{ }^{60} \mathrm{Co}=1.2 \mathrm{E}+2 \\
{ }^{137} \mathrm{Cs}=8.8 \mathrm{E}+5 \\
\text { Density }=1.095 \\
\text { G-Alpha }=3.1 \mathrm{E}+3 \\
\text { Total activity }=1.3 \mathrm{E}+6\end{array}$ \\
\hline
\end{tabular}


SAMPLE ANALYSIS DATA SUMMARY FOR ALL GAAT W-9 SLUDGE SAMPLES (Continued)

\begin{tabular}{|c|c|c|c|c|c|c|c|}
\hline \multicolumn{4}{|c|}{ Sample } & \multirow[b]{2}{*}{$\begin{array}{l}\text { PulsAir and Flygt } \\
\text { mixer status }\end{array}$} & \multirow[b]{2}{*}{$\begin{array}{c}\text { SCS } \\
\text { status }\end{array}$} & \multirow[b]{2}{*}{$\begin{array}{c}\text { Analysis } \\
\text { requested }^{b}\end{array}$} & \multirow[b]{2}{*}{$\begin{array}{c}\text { Data } \\
\text { summary }^{c}\end{array}$} \\
\hline $\begin{array}{c}\text { Collection } \\
\text { date }\end{array}$ & No. & Type & Location $^{a}$ & & & & \\
\hline $5-26-99$ & $\begin{array}{l}\text { GAAT- } \\
\text { W9-030 }\end{array}$ & Grab & $\begin{array}{l}\text { Isolok sampler, } \\
\text { SP-3 }\end{array}$ & $\begin{array}{l}\text { Supply tank at } \sim 35 \mathrm{psig} \\
\text { Dwell time, } 8 \mathrm{~s} \\
\text { Injection time, } 1.5 \mathrm{~s} \\
\text { Total run time, } 4.5 \mathrm{~h}\end{array}$ & $\begin{array}{l}\text { DiscFlo } \\
\text { pump } \\
\text { speed }=34 \\
\mathrm{~Hz}\end{array}$ & $\begin{array}{l}\text { G-alpha, G-beta, } \\
\text { beta, gamma, } \\
\text { alpha scan, } \\
\text { density, TS, TSS, } \\
\text { TSS50 }\end{array}$ & $\begin{array}{l}{ }^{238} \mathrm{U}=20.3 \% \\
{ }^{233} \mathrm{U} /{ }^{234} \mathrm{U}=22.5 \% \\
{ }^{239} \mathrm{Pu} /{ }^{240} \mathrm{Pu}=12.7 \% \\
{ }^{238} \mathrm{Pu} /{ }^{241} \mathrm{Am}=13.2 \% \\
{ }^{244} \mathrm{Cm}=31.4 \% \\
{ }^{60} \mathrm{Co}=4.8 \mathrm{E}+1 \\
{ }^{137} \mathrm{Cs}=1.4 \mathrm{E}+5 \\
\text { Density }=1.024 \\
\text { G-Alpha }=3.9 \mathrm{E}+2 \\
\text { G-Beta }=2.0 \mathrm{E}+5 \\
\text { TS }=28,200 \\
\text { TSS }=12,000 \\
\text { TSS50 }=<10\end{array}$ \\
\hline $5-26-99$ & $\begin{array}{l}\text { GAAT- } \\
\text { W9-031 }\end{array}$ & Grab & $\begin{array}{l}\text { Isolok sampler, } \\
\text { SP-2 }\end{array}$ & $\begin{array}{l}\text { Supply tank at } \sim 35 \mathrm{psig} \\
\text { Dwell time, } 8 \mathrm{~s} \\
\text { Injection time, } 1.5 \mathrm{~s} \\
\text { Total run time, } 4.5 \mathrm{~h}\end{array}$ & $\begin{array}{l}\text { DiscFlo } \\
\text { pump speed } \\
=34 \mathrm{~Hz}\end{array}$ & $\begin{array}{l}\text { G-alpha, G-beta, } \\
\text { beta, gamma, } \\
\text { alpha scan, } \\
\text { density, TS, TSS, } \\
\text { TSS50 }\end{array}$ & $\begin{array}{l}{ }^{238} \mathrm{U}=18.7 \% \\
{ }^{233} \mathrm{U}{ }^{234} \mathrm{U}=22.8 \% \\
{ }^{239} \mathrm{Pu} /{ }^{240} \mathrm{Pu}=11.3 \% \\
{ }^{238} \mathrm{Pu} /{ }^{241} \mathrm{Am}=13.6 \% \\
{ }^{244} \mathrm{Cm}=33.5 \% \\
{ }^{60} \mathrm{Co}=4.8 \mathrm{E}+1 \\
{ }^{137} \mathrm{Cs}=1.5 \mathrm{E}+5 \\
\text { Density }=1.028 \\
\text { G-Alpha }=4.3 \mathrm{E}+2 \\
\text { G-Beta }=2.2 \mathrm{E}+5 \\
\text { TS }=31,200 \\
\text { TSS }=12,800 \\
\text { TSS50 }=<10\end{array}$ \\
\hline
\end{tabular}


SAMPLE ANALYSIS DATA SUMMARY FOR ALL GAAT W-9 SLUDGE SAMPLES (Continued)

\begin{tabular}{|c|c|c|c|c|c|c|c|}
\hline \multicolumn{4}{|c|}{ Sample } & \multirow[b]{2}{*}{$\begin{array}{l}\text { PulsAir and Flygt } \\
\text { mixer status }\end{array}$} & \multirow[b]{2}{*}{$\begin{array}{l}\text { SCS } \\
\text { status }\end{array}$} & \multirow[b]{2}{*}{$\begin{array}{c}\text { Analysis } \\
\text { requested }^{b}\end{array}$} & \multirow[b]{2}{*}{$\begin{array}{c}\text { Data } \\
\text { summary }^{c}\end{array}$} \\
\hline $\begin{array}{c}\text { Collection } \\
\text { date }\end{array}$ & No. & Type & Location $^{a}$ & & & & \\
\hline $6-8-99$ & $\begin{array}{l}\text { GAAT- } \\
\text { W9-032 }\end{array}$ & Grab & $\begin{array}{l}\text { Isolok sampler, } \\
\text { SP-2 }\end{array}$ & $\begin{array}{l}\text { Supply tank at } \sim 35 \text { psig } \\
\text { Dwell time, } 8 \mathrm{~s} \\
\text { Injection time, } 1 \mathrm{~s} \\
\text { Total run time, } 4 \mathrm{~h}\end{array}$ & $\begin{array}{l}\text { DiscFlo } \\
\text { pump Speed } \\
=34 \mathrm{~Hz}\end{array}$ & $\begin{array}{l}\text { G-alpha, G-beta, } \\
\text { beta, gamma, } \\
\text { alpha scan, } \\
\text { density, TS, TSS, } \\
\text { TSS50 }\end{array}$ & $\begin{array}{l}{ }^{238} \mathrm{U}=0.8 \% \\
{ }^{233} \mathrm{U} /{ }^{234} \mathrm{U}=2.0 \% \\
{ }^{239} \mathrm{Pu} /{ }^{240} \mathrm{Pu}=4.3 \% \\
{ }^{238} \mathrm{Pu} /{ }^{241} \mathrm{Am}=12.0 \% \\
{ }^{244} \mathrm{Cm}=80.8 \% \\
{ }^{60} \mathrm{Co}=5.9 \mathrm{E}+2 \\
{ }^{137} \mathrm{Cs}=1.8 \mathrm{E}+5 \\
\text { Density }=1.037 \\
{ }^{152} \mathrm{Eu}=6.8 \mathrm{E}+2 \\
{ }^{155} \mathrm{Eu}=5.5 \mathrm{E}+2 \\
\mathrm{G}-\mathrm{Alpha}=9.1 \mathrm{E}+3 \\
\mathrm{G}-\mathrm{Beta}=9.9 \mathrm{E}+5 \\
\mathrm{TS}=38,000 \\
\mathrm{TSS}=18,700 \\
\mathrm{TSS} 50=370\end{array}$ \\
\hline $6-9-99$ & $\begin{array}{l}\text { GAAT- } \\
\text { W9-033 }\end{array}$ & Grab & $\begin{array}{l}\text { NE Riser, 4-ft } \\
\text { below } \\
\text { supernate } \\
\text { level } \\
\text { Tank volume = } \\
\sim 135,506 \text { gal }\end{array}$ & $\begin{array}{l}\text { Supply tank at } \sim 35 \mathrm{psig} \\
\text { Dwell time, } 8 \mathrm{~s} \\
\text { Injection time, } 1.5 \mathrm{~s} \\
\text { Total run time, } 28.5 \mathrm{~h}\end{array}$ & $\begin{array}{l}\text { DiscFlo } \\
\text { pump speed } \\
=34 \mathrm{~Hz}, \\
\text { (Two } \\
\text { operations, } \\
\text { see logbook } \\
\text { dated 6-8 \& } \\
\text { 6-9-99) } \\
\end{array}$ & Density, TS, TSS & $\begin{array}{l}\text { Density }=1.027 \\
\text { TS }=37,500 \\
\text { TSS }=18,900\end{array}$ \\
\hline
\end{tabular}


SAMPLE ANALYSIS DATA SUMMARY FOR ALL GAAT W-9 SLUDGE SAMPLES (Continued)

\begin{tabular}{|c|c|c|c|c|c|c|c|}
\hline \multicolumn{4}{|c|}{ Sample } & \multirow[b]{2}{*}{$\begin{array}{l}\text { PulsAir and Flygt } \\
\text { mixer status }\end{array}$} & \multirow[b]{2}{*}{$\begin{array}{l}\text { SCS } \\
\text { status }\end{array}$} & \multirow[b]{2}{*}{$\begin{array}{c}\text { Analysis } \\
\text { requested }^{b}\end{array}$} & \multirow[b]{2}{*}{$\begin{array}{c}\text { Data } \\
\text { summary }^{c}\end{array}$} \\
\hline $\begin{array}{c}\text { Collection } \\
\text { date }\end{array}$ & No. & Type & Location $^{a}$ & & & & \\
\hline $6-11-99$ & $\begin{array}{l}\text { GAAT- } \\
\text { W9-034 }\end{array}$ & Grab & $\begin{array}{l}\text { Isolok sampler, } \\
\text { SP-1 }\end{array}$ & $\begin{array}{l}\text { Supply tank at } \sim 35 \mathrm{psig} \\
\text { Dwell time, } 8 \mathrm{~s} \\
\text { Injection time, } 1 \mathrm{~s} \\
\text { Total run time, } 78 \mathrm{~h}\end{array}$ & $\begin{array}{l}\text { DiscFlo } \\
\text { pump Speed } \\
=34 \mathrm{~Hz} \\
\text { (Two } \\
\text { operations, } \\
\text { see logbook } \\
\text { dated 6-11- } \\
\text { 99) }\end{array}$ & $\begin{array}{l}\text { Density, TS, TDS, } \\
\text { TSS }\end{array}$ & $\begin{array}{l}\text { Density }=1.031 \\
\text { TDS }=18,300 \\
\text { TS }=38,400 \\
\text { TSS }=20,100\end{array}$ \\
\hline $6-15-99$ & $\begin{array}{l}\text { GAAT- } \\
\text { W9-035 }\end{array}$ & Grab & $\begin{array}{l}\text { NE Riser, 4-ft } \\
\text { below } \\
\text { supernate } \\
\text { level } \\
\text { Tank volume = } \\
\sim 122,719 \text { gal }\end{array}$ & $\begin{array}{l}\text { Supply tank at } \sim 20 \mathrm{psig} \\
\text { Dwell time, } 8 \mathrm{~s} \\
\text { Injection time, } 1 \mathrm{~s} \\
\text { Total run time, } 6 \mathrm{~h}\end{array}$ & $\begin{array}{l}\text { Normal } \\
\text { PulsAir } \\
\text { system } \\
\text { operation }\end{array}$ & $\begin{array}{l}\text { G-alpha, G-beta, } \\
\text { beta, gamma, } \\
\text { alpha scan, } \\
\text { density, TS, TSS, } \\
\text { Total activity }\end{array}$ & $\begin{array}{l}{ }^{238} \mathrm{U}=0.7 \% \\
{ }^{233} \mathrm{U} /{ }^{234} \mathrm{U}=2.0 \% \\
{ }^{239} \mathrm{Pu} /{ }^{240} \mathrm{Pu}=5.8 \% \\
{ }^{238} \mathrm{Pu} /{ }^{241} \mathrm{Am}=16.5 \% \\
{ }^{244} \mathrm{Cm}=75.0 \% \\
{ }^{60} \mathrm{Co}=1.3 \mathrm{E}+3 \\
{ }^{137} \mathrm{Cs}=2.7 \mathrm{E}+5 \\
\text { Density }=1.037 \\
{ }^{152} \mathrm{Eu}=1.3 \mathrm{E}+3 \\
{ }^{154} \mathrm{Eu}=1.2 \mathrm{E}+3 \\
\mathrm{G}-\mathrm{Alpha}=2.1 \mathrm{E}+4 \\
\text { TS }=55,900 \\
\text { TSS }=36,700 \\
\text { Total activity }=2.0 \mathrm{E}+6\end{array}$ \\
\hline $6-16-99$ & $\begin{array}{l}\text { GAAT- } \\
\text { W9-038 }\end{array}$ & Grab & $\begin{array}{l}\text { NE Riser, 4-ft } \\
\text { below } \\
\text { supernate } \\
\text { level } \\
\text { Tank volume = } \\
\sim 130,803 \text { gal }\end{array}$ & $\begin{array}{l}\text { Supply tank@ } \sim 35 \mathrm{psig} \\
\text { Dwell time, } 8 \mathrm{~s} \\
\text { Injection time, } 1 \mathrm{~s} \\
\text { Total run time, } 10.5 \mathrm{~h}\end{array}$ & $\begin{array}{l}\text { Normal } \\
\text { system } \\
\text { operation }\end{array}$ & Density, TS, TSS & $\begin{array}{l}\text { Density }=1.041 \\
\text { TS }=58,600 \\
\text { TSS }=39,700\end{array}$ \\
\hline
\end{tabular}


SAMPLE ANALYSIS DATA SUMMARY FOR ALL GAAT W-9 SLUDGE SAMPLES (Continued)

\begin{tabular}{|c|c|c|c|c|c|c|c|}
\hline \multicolumn{4}{|c|}{ Sample } & \multirow[b]{2}{*}{$\begin{array}{l}\text { PulsAir and Flygt } \\
\text { mixer status }\end{array}$} & \multirow[b]{2}{*}{$\begin{array}{c}\text { SCS } \\
\text { status }\end{array}$} & \multirow[b]{2}{*}{$\begin{array}{c}\text { Analysis } \\
\text { requested }^{b}\end{array}$} & \multirow[b]{2}{*}{$\begin{array}{c}\text { Data } \\
\text { summary }^{c}\end{array}$} \\
\hline $\begin{array}{c}\text { Collection } \\
\text { date }\end{array}$ & No. & Type & Location $^{a}$ & & & & \\
\hline $6-17-99$ & $\begin{array}{l}\text { GAAT- } \\
\text { W9-039 }\end{array}$ & Grab & Isolok sampler & $\begin{array}{l}\text { Supply tank at } \sim 35 \mathrm{psig} \\
\text { Dwell time, } 8 \mathrm{~s} \\
\text { Injection time, } 1.0 \mathrm{~s} \\
\text { Total run time, } 7 \mathrm{~h}\end{array}$ & $\begin{array}{l}\text { Normal } \\
\text { system } \\
\text { operation }\end{array}$ & Density, TS, TSS & $\begin{array}{l}\text { Density }=1.048 \\
\text { TS }=57,200 \\
\text { TSS }=37,600\end{array}$ \\
\hline $6-17-99$ & $\begin{array}{l}\text { GAAT- } \\
\text { W9-040 }\end{array}$ & Grab & Isolok sampler & $\begin{array}{l}\text { Supply tank at } \sim 35 \mathrm{psig} \\
\text { Dwell time, } 8 \mathrm{~s} \\
\text { Injection time, } 1 \mathrm{~s} \\
\text { Total run time, } 10.5 \mathrm{~h}\end{array}$ & $\begin{array}{l}\text { Normal } \\
\text { system } \\
\text { operation }\end{array}$ & Density, TS, TSS & $\begin{array}{l}\text { Density }=1.041 \\
\text { TS }=56,600 \\
\text { TSS }=37,200\end{array}$ \\
\hline $6-22-99$ & $\begin{array}{l}\text { GAAT- } \\
\text { W9-041 }\end{array}$ & Grab & $\begin{array}{l}\text { Isolok sampler, } \\
\text { SP-2 }\end{array}$ & $\begin{array}{l}\text { Supply tank at } \sim 20 \mathrm{psig} \\
\text { Dwell time, } 8 \mathrm{~s} \\
\text { Injection time, } 0.7 \mathrm{~s} \\
\text { Total run time, } 1.5 \mathrm{~h}\end{array}$ & $\begin{array}{l}\text { Normal } \\
\text { system } \\
\text { operation }\end{array}$ & Density, TS, TSS & $\begin{array}{l}\text { Density }=1.044 \\
\text { pH }=10.10 \\
\text { TS }=55,500 \\
\text { TSS }=35,900\end{array}$ \\
\hline 6-30-99 & $\begin{array}{l}\text { GAAT- } \\
\text { W9-042 }\end{array}$ & Grab & $\begin{array}{l}\text { Isolok sampler, } \\
\text { SP-1 }\end{array}$ & $\begin{array}{l}\text { Supply tank at } \sim 26 \mathrm{psig} \\
\text { Dwell time, } 12 \mathrm{~s} \\
\text { Injection time, } 1 \mathrm{~s} \\
\text { Total run time, } 5 \mathrm{~h}\end{array}$ & $\begin{array}{l}\text { Normal } \\
\text { system } \\
\text { operation }\end{array}$ & $\begin{array}{l}\text { See GAAT } \\
\text { Sampling and } \\
\text { Analysis Plan }\end{array}$ & $\begin{array}{l}\text { Density }=1.042 \\
\text { TS }=51,700 \\
\text { TSS }=33,400\end{array}$ \\
\hline
\end{tabular}


SAMPLE ANALYSIS DATA SUMMARY FOR ALL GAAT W-9 SLUDGE SAMPLES (Continued)

\begin{tabular}{|c|c|c|c|c|c|c|c|}
\hline \multicolumn{4}{|c|}{ Sample } & \multirow[b]{2}{*}{$\begin{array}{l}\text { PulsAir and Flygt } \\
\text { mixer status }\end{array}$} & \multirow[b]{2}{*}{$\begin{array}{l}\text { SCS } \\
\text { status }\end{array}$} & \multirow[b]{2}{*}{$\begin{array}{c}\text { Analysis } \\
\text { requested }^{b}\end{array}$} & \multirow[b]{2}{*}{$\begin{array}{c}\text { Data } \\
\text { summary }^{c}\end{array}$} \\
\hline $\begin{array}{c}\text { Collection } \\
\text { date }\end{array}$ & No. & Type & Location $^{a}$ & & & & \\
\hline $7-15-99$ & $\begin{array}{l}\text { GAAT- } \\
\text { W9-043 }\end{array}$ & Grab & $\begin{array}{l}\text { Isolok sampler, } \\
\text { SP-2 }\end{array}$ & $\begin{array}{l}\text { Supply tank at } \sim 30 \mathrm{psig} \\
\text { Dwell time, } 12 \mathrm{~s} \\
\text { Injection time, } 0.5 \mathrm{~s} \\
\text { Total run time, } 6.5 \mathrm{~h}\end{array}$ & $\begin{array}{l}\text { Normal } \\
\text { system } \\
\text { operation }\end{array}$ & $\begin{array}{l}\text { See GAAT } \\
\text { Sampling and } \\
\text { Analysis Plan }\end{array}$ & $\begin{array}{l}{ }^{238} \mathrm{U}=1.3 \% \\
{ }^{233} \mathrm{U} /{ }^{234} \mathrm{U}=2.0 \% \\
{ }^{239} \mathrm{Pu} /{ }^{240} \mathrm{Pu}=7.4 \% \\
{ }^{238} \mathrm{Pu} /{ }^{241} \mathrm{Am}=13.5 \% \\
{ }^{244} \mathrm{Cm}=75.8 \% \\
{ }^{60} \mathrm{Co}=7.9 \mathrm{E}+2 \\
{ }^{137} \mathrm{Cs}=2.1 \mathrm{E}+5 \\
\text { Density = 1.039 } \\
{ }^{152} \mathrm{Eu}=7.1 \mathrm{E}+2 \\
{ }^{154} \mathrm{Eu}=1.0 \mathrm{E}+3 \\
{ }^{155} \mathrm{Eu}=0.8 \mathrm{E}+2 \\
\mathrm{G}-\mathrm{Alpha}=1.5 \mathrm{E}+4 \\
\mathrm{G}-\mathrm{Beta}=1.3 \mathrm{E}+6 \\
\mathrm{pH}=9.93 \\
\text { Total activity }=1.3 \mathrm{E}+6 \\
\text { TS }=44,400 \\
\text { TSS }=25,800\end{array}$ \\
\hline $7-19-99$ & $\begin{array}{l}\text { GAAT- } \\
\text { W9-044 }\end{array}$ & Grab & $\begin{array}{l}\text { Isolok sampler, } \\
\text { SP-1 }\end{array}$ & $\begin{array}{l}\text { Supply tank at } \sim 33 \text { psig } \\
\text { Dwell time, } 12 \mathrm{~s} \\
\text { Injection time, } 0.5 \mathrm{~s} \\
\text { Total run time, } 7 \mathrm{~h}\end{array}$ & $\begin{array}{l}\text { Normal } \\
\text { system } \\
\text { operation }\end{array}$ & $\begin{array}{l}\text { See GAAT } \\
\text { Sampling and } \\
\text { Analysis Plan }\end{array}$ & $\begin{array}{l}{ }^{238} \mathrm{U}=0.7 \% \\
{ }^{233} \mathrm{U} /{ }^{234} \mathrm{U}=2.0 \% \\
{ }^{239} \mathrm{Pu} /{ }^{240} \mathrm{Pu}=4.8 \% \\
{ }^{238} \mathrm{Pu} /{ }^{241} \mathrm{Am}=17.2 \% \\
{ }^{244} \mathrm{Cm}=75.4 \% \\
{ }^{60} \mathrm{Co}=1.6 \mathrm{E}+3 \\
{ }^{137} \mathrm{Cs}=2.3 \mathrm{E}+5 \\
\text { Density }=1.057 \\
{ }^{152} \mathrm{Eu}=1.3 \mathrm{E}+3 \\
{ }^{154} \mathrm{Eu}=1.5 \mathrm{E}+3 \\
\mathrm{G}-\mathrm{Alpha}=2.1 \mathrm{E}+4 \\
\mathrm{G}-\mathrm{Beta}=1.8 \mathrm{E}+6 \\
\text { Total activity }=1.3 \mathrm{E}+6 \\
\mathrm{TS}=50,200 \\
\mathrm{TSS}=33,300\end{array}$ \\
\hline
\end{tabular}


SAMPLE ANALYSIS DATA SUMMARY FOR ALL GAAT W-9 SLUDGE SAMPLES (Continued)

\begin{tabular}{|c|c|c|c|c|c|c|c|}
\hline \multicolumn{4}{|c|}{ Sample } & \multirow[b]{2}{*}{$\begin{array}{c}\text { PulsAir and Flygt } \\
\text { mixer status }\end{array}$} & \multirow[b]{2}{*}{$\begin{array}{c}\text { SCS } \\
\text { status }\end{array}$} & \multirow[b]{2}{*}{$\begin{array}{c}\text { Analysis } \\
\text { requested }^{b}\end{array}$} & \multirow[b]{2}{*}{$\begin{array}{c}\text { Data } \\
\text { summary }^{c}\end{array}$} \\
\hline $\begin{array}{c}\text { Collection } \\
\text { date }\end{array}$ & No. & Type & Location $^{a}$ & & & & \\
\hline $7-22-99$ & $\begin{array}{l}\text { GAAT- } \\
\text { W9-045 }\end{array}$ & Grab & $\begin{array}{l}\text { Isolok sampler, } \\
\text { SP-2 }\end{array}$ & $\begin{array}{l}\text { Supply tank at } \sim 30 \mathrm{psig} \\
\text { Dwell time, } 12 \mathrm{~s} \\
\text { Injection time, } 0.5 \mathrm{~s} \\
\text { Total run time, } 32 \mathrm{~h}\end{array}$ & $\begin{array}{l}\text { Normal } \\
\text { system } \\
\text { operation }\end{array}$ & $\begin{array}{l}\text { Density, pH, TS, } \\
\text { TSS }\end{array}$ & $\begin{array}{l}\text { Density }=1.044 \\
\mathrm{pH}=9.93 \\
\mathrm{TS}=55,500 \\
\mathrm{TSS}=39,800\end{array}$ \\
\hline $7-28-99$ & $\begin{array}{l}\text { GAAT- } \\
\text { W9-046 }\end{array}$ & Grab & $\begin{array}{l}\text { Isolok sampler, } \\
\text { SP-2 }\end{array}$ & $\begin{array}{l}\text { Supply tank at } \sim 38 \mathrm{psig} \\
\text { Dwell time, } 8 \mathrm{~s} \\
\text { Injection time, } 1 \mathrm{~s} \\
\text { Total run time, } 9.5 \mathrm{~h} \\
\text { Dwell time, } 12 \mathrm{~s} \\
\text { Injection time, } 0.5 \mathrm{~s} \\
\text { Total run time, } 7 \mathrm{~h} \\
\end{array}$ & $\begin{array}{l}\text { SCS transfer } \\
\text { mode }\end{array}$ & $\begin{array}{l}\text { Density, pH, TS, } \\
\text { TSS }\end{array}$ & $\begin{array}{l}\text { Density }=1.058 \\
\text { pH }=9.85 \\
\text { TS }=52,600 \\
\text { TSS }=33,200\end{array}$ \\
\hline $8-10-99$ & $\begin{array}{l}\text { GAAT- } \\
\text { W9-047 }\end{array}$ & Grab & $\begin{array}{l}\text { N Riser } \\
20 \mathrm{ft} 4 \text { in }- \\
21 \mathrm{ft} 10 \mathrm{in} \\
\text { depth }\end{array}$ & Systems not on-line & $\begin{array}{l}\text { Systems not } \\
\text { on-line }\end{array}$ & Density, TS, TSS & $\begin{array}{l}\text { Density }=1.05 \\
\text { TS }=68,600 \\
\text { TSS }=52,800\end{array}$ \\
\hline $8-10-99$ & $\begin{array}{l}\text { GAAT- } \\
\text { W9-048 }\end{array}$ & Grab & $\begin{array}{l}\text { N Riser } \\
21 \mathrm{ft} 10 \text { in }-23 \\
\mathrm{ft} 4 \text { in depth }\end{array}$ & Systems not on-line & $\begin{array}{l}\text { Systems not } \\
\text { on-line }\end{array}$ & Density, TS, TSS & $\begin{array}{l}\text { Density }=1.09 \\
\mathrm{TS}=13.3 \% \\
\mathrm{TSS}=116,000\end{array}$ \\
\hline $8-10-99$ & $\begin{array}{l}\text { GAAT- } \\
\text { W9-049 }\end{array}$ & Grab & $\begin{array}{l}\text { N Riser } \\
23 \mathrm{ft} 4 \text { in }-24 \\
\mathrm{ft} 10 \text { in depth } \\
\end{array}$ & Systems not on-line & $\begin{array}{l}\text { Systems not } \\
\text { on-line }\end{array}$ & $\begin{array}{l}\text { Density, TS, TSS, } \\
\text { ICP Metals (See } \\
\text { analysis report) } \\
\end{array}$ & $\begin{array}{l}\text { Density }=1.17 \\
\text { TS }=20.2 \% \\
\text { TSS }=192,000 \\
\end{array}$ \\
\hline $8-17-99$ & $\begin{array}{l}\text { GAAT- } \\
\text { W9-050 }\end{array}$ & Grab & $\begin{array}{l}\text { Isolok sampler, } \\
\text { SP-2 }\end{array}$ & $\begin{array}{l}\text { Supply tank at } \sim 50 \mathrm{psig} \\
\text { Dwell time, } 12 \mathrm{~s} \\
\text { Injection time, } 0.5 \mathrm{~s} \\
\text { Total run time, } 103 \mathrm{~h}\end{array}$ & $\begin{array}{l}\text { SCS transfer } \\
\text { mode }\end{array}$ & $\begin{array}{l}\text { Density, pH, TS, } \\
\text { TSS }\end{array}$ & $\begin{array}{l}\text { Density }=1.05 \\
\text { pH }=9.92 \\
\text { TS }=49,600 \\
\text { TSS }=30,800\end{array}$ \\
\hline $9-9-99$ & $\begin{array}{l}\text { GAAT- } \\
\text { W9-051 }\end{array}$ & Grab & $\begin{array}{l}\text { Isolok sampler, } \\
\text { SP-2 }\end{array}$ & $\begin{array}{l}\text { Supply tank at } \sim 48 \mathrm{psig} \\
\text { Dwell time, } 14 \mathrm{~s} \\
\text { Injection time, } 0.5 \mathrm{~s} \\
\text { Total run time, } 151 \mathrm{~h}\end{array}$ & $\begin{array}{l}\text { SCS transfer } \\
\text { mode }\end{array}$ & $\begin{array}{l}\text { Density, pH, TS, } \\
\text { TSS }\end{array}$ & $\begin{array}{l}\text { Density }=1.023 \\
\mathrm{pH}=9.92 \\
\mathrm{TS}=56,100 \\
\mathrm{TSS}=38,400\end{array}$ \\
\hline
\end{tabular}


SAMPLE ANALYSIS DATA SUMMARY FOR ALL GAAT W-9 SLUDGE SAMPLES (Continued)

\begin{tabular}{|c|c|c|c|c|c|c|c|}
\hline \multicolumn{4}{|c|}{ Sample } & \multirow[b]{2}{*}{$\begin{array}{l}\text { PulsAir and Flygt } \\
\text { mixer status }\end{array}$} & \multirow[b]{2}{*}{$\begin{array}{l}\text { SCS } \\
\text { status }\end{array}$} & \multirow[b]{2}{*}{$\begin{array}{c}\text { Analysis } \\
\text { requested }^{b}\end{array}$} & \multirow[b]{2}{*}{$\begin{array}{c}\text { Data } \\
\text { summary }^{c}\end{array}$} \\
\hline $\begin{array}{c}\text { Collection } \\
\text { date }\end{array}$ & No. & Type & Location $^{a}$ & & & & \\
\hline $9-20-99$ & $\begin{array}{l}\text { GAAT- } \\
\text { W9-052 }\end{array}$ & Grab & $\begin{array}{l}\text { Isolok sampler, } \\
\text { SP-1 }\end{array}$ & $\begin{array}{l}\text { Supply tank at } \sim 48 \mathrm{psig} \\
\text { Dwell time, } 12 \mathrm{~s} \\
\text { Injection time, } 0.5 \mathrm{~s} \\
\text { Total run time, } 5.5 \mathrm{~h} \\
\text { Flygt mixer tests }\end{array}$ & $\begin{array}{l}\text { Normal } \\
\text { system } \\
\text { operation }\end{array}$ & $\begin{array}{l}\text { Density, pH, TS, } \\
\text { TSS, TSS50 }\end{array}$ & $\begin{array}{l}\text { Density }=1.045 \\
\text { pH }=9.73 \\
\text { TS }=59,100 \\
\text { TSS }=40,300 \mathrm{~L}\end{array}$ \\
\hline $9-20-99$ & $\begin{array}{l}\text { GAAT- } \\
\text { W9-053 }\end{array}$ & Grab & $\begin{array}{l}\text { Isolok sampler, } \\
\text { SP-2 }\end{array}$ & $\begin{array}{l}\text { Supply tank at } \sim 48 \mathrm{psig} \\
\text { Dwell time, } 12 \mathrm{~s} \\
\text { Injection time, } 0.5 \mathrm{~s} \\
\text { Total run time, } 5.5 \mathrm{~h} \\
\text { Flygt mixer tests }\end{array}$ & $\begin{array}{l}\text { Normal } \\
\text { system } \\
\text { operation }\end{array}$ & $\begin{array}{l}\text { Density, pH, TS, } \\
\text { TSS, TSS50 }\end{array}$ & $\begin{array}{l}\text { Density }=1.047 \\
\text { pH }=9.76 \\
\text { TS }=64,000 \\
\text { TSS }=44,300\end{array}$ \\
\hline $9-23-99$ & $\begin{array}{l}\text { GAAT- } \\
\text { W9-054 }\end{array}$ & Grab & $\begin{array}{l}\text { Isolok sampler, } \\
\text { SP-1 }\end{array}$ & $\begin{array}{l}\text { Supply tank at } \sim 48 \text { psig } \\
\text { Dwell time, } 12 \mathrm{~s} \\
\text { Injection time, } 0.5 \mathrm{~s} \\
\text { Total run time, } 75 \mathrm{~h} \\
\text { Flygt mixer tests }\end{array}$ & $\begin{array}{l}\text { Normal } \\
\text { system } \\
\text { operation }\end{array}$ & $\begin{array}{l}\text { Density, } \mathrm{pH}, \mathrm{TS}, \\
\text { TSS, micron filter }\end{array}$ & $\begin{array}{l}\text { Density }=1.039 \\
\mathrm{pH}=9.90 \\
\mathrm{TS}=38,500 \\
\mathrm{TSS}=19,600 \\
\text { Micron filter }=60\end{array}$ \\
\hline $9-23-99$ & $\begin{array}{l}\text { GAAT- } \\
\text { W9-055 }\end{array}$ & Grab & $\begin{array}{l}\text { Isolok sampler, } \\
\text { SP-2 }\end{array}$ & $\begin{array}{l}\text { Supply tank at } \sim 48 \text { psig } \\
\text { Dwell time, } 12 \mathrm{~s} \\
\text { Injection time, } 0.5 \mathrm{~s} \\
\text { Total run time, } 75 \mathrm{~h} \\
\text { Flygt mixer tests }\end{array}$ & $\begin{array}{l}\text { Normal } \\
\text { system } \\
\text { operation }\end{array}$ & $\begin{array}{l}\text { Density, } \mathrm{pH}, \mathrm{TS}, \\
\text { TSS, micron filter }\end{array}$ & $\begin{array}{l}\text { Density }=1.037 \\
\text { pH }=9.92 \\
\text { TS }=43,900 \\
\text { TSS }=24,800 \\
\text { Micron filter }=1,050\end{array}$ \\
\hline $9-28-99$ & $\begin{array}{l}\text { GAAT- } \\
\text { W9-056 }\end{array}$ & Grab & $\begin{array}{l}\text { Isolok sampler, } \\
\text { SP-2 }\end{array}$ & $\begin{array}{l}\text { Supply tank at } \sim 48 \mathrm{psig} \\
\text { Dwell time, } 12 \mathrm{~s} \\
\text { Injection time, } 0.5 \mathrm{~s} \\
\text { Total run time, } 198 \mathrm{~h} \\
\text { Flygt mixer on } \sim 6.5 \mathrm{~h} \\
\end{array}$ & $\begin{array}{l}\text { Normal } \\
\text { system } \\
\text { operation }\end{array}$ & $\begin{array}{l}\text { Density, pH, TS, } \\
\text { TSS, TSS50 }\end{array}$ & $\begin{array}{l}\text { Density }=1.039 \\
\text { pH }=9.90 \\
\text { TS }=38,500 \\
\text { TSS }=19,600 \\
\text { TSS50 }=460\end{array}$ \\
\hline $10-26-99$ & $\begin{array}{l}\text { GAAT- } \\
\text { W9-057 }\end{array}$ & Grab & $\begin{array}{l}\text { Isolok sampler, } \\
\text { SP-2 }\end{array}$ & $\begin{array}{l}\text { Supply tank at } \sim 48 \mathrm{psig} \\
\text { Dwell time, } 14 \mathrm{~s} \\
\text { Injection time, } 0.5 \mathrm{~s} \\
\text { Total run time, } \sim 99 \mathrm{~h} \\
\text { Flygt mixer on } \sim 30 \mathrm{~h}\end{array}$ & $\begin{array}{l}\text { Normal } \\
\text { system } \\
\text { operation }\end{array}$ & $\begin{array}{l}\text { Density, pH, TS, } \\
\text { TSS, TSS50 }\end{array}$ & $\begin{array}{l}\text { Density }=1.055 \\
\text { pH }=9.86 \\
\text { TS }=68,400 \\
\text { TSS }=49,800 \\
\text { TSS50 }=1,000\end{array}$ \\
\hline
\end{tabular}


SAMPLE ANALYSIS DATA SUMMARY FOR ALL GAAT W-9 SLUDGE SAMPLES (Continued)

\begin{tabular}{|c|c|c|c|c|c|c|c|}
\hline \multicolumn{4}{|c|}{ Sample } & \multirow[b]{2}{*}{$\begin{array}{l}\text { PulsAir and Flygt } \\
\text { mixer status }\end{array}$} & \multirow[b]{2}{*}{$\begin{array}{l}\text { SCS } \\
\text { status }\end{array}$} & \multirow[b]{2}{*}{$\begin{array}{c}\text { Analysis } \\
\text { requested }^{b}\end{array}$} & \multirow[b]{2}{*}{$\begin{array}{c}\text { Data } \\
\text { summary }\end{array}$} \\
\hline $\begin{array}{c}\text { Collection } \\
\text { date }\end{array}$ & No. & Type & Location $^{a}$ & & & & \\
\hline $11-17-99$ & $\begin{array}{l}\text { GAAT- } \\
\text { W9-058 }\end{array}$ & Grab & $\begin{array}{l}\text { Isolok sampler, } \\
\text { SP-2 }\end{array}$ & $\begin{array}{l}\text { Supply tank at } \sim 60 \mathrm{psig} \\
\text { Dwell time, } 14 \mathrm{~s} \\
\text { Injection time, } 0.5 \mathrm{~s} \\
\text { Total run time, } \sim 456 \mathrm{~h} \\
\text { Flygt mixer on } \sim 24 \mathrm{~h}\end{array}$ & $\begin{array}{l}\text { Normal } \\
\text { system } \\
\text { operation }\end{array}$ & $\begin{array}{l}\text { Density, pH, TS, } \\
\text { TSS, TSS50 }\end{array}$ & $\begin{array}{l}\text { Density }=1.028 \\
\text { pH }=9.85 \\
\text { TS }=56,400 \\
\text { TSS }=39,300 \\
\text { TSS50 }=860\end{array}$ \\
\hline $12-7-99$ & $\begin{array}{l}\text { GAAT- } \\
\text { W9-059 }\end{array}$ & Grab & $\begin{array}{l}\text { Isolok sampler, } \\
\text { SP-2 }\end{array}$ & $\begin{array}{l}\text { Supply tank at } \sim 60 \mathrm{psig} \\
\text { Dwell time, } 12 \mathrm{~s} \\
\text { Injection time, } 0.5 \mathrm{~s} \\
\text { Total run time, } \sim 144 \mathrm{~h} \\
\text { Flygt mixer not started } \\
\text { until transfer date }\end{array}$ & $\begin{array}{l}\text { Normal } \\
\text { system } \\
\text { operation }\end{array}$ & $\begin{array}{l}\text { Density, pH, TS, } \\
\text { TSS, TSS50 }\end{array}$ & $\begin{array}{l}\text { Density }=1.024 \\
\text { pH }=9.77 \\
\text { TS }=51,500 \\
\text { TSS }=36,400 \\
\text { TSS50 }=180\end{array}$ \\
\hline $1-13-00$ & $\begin{array}{l}\text { GAAT- } \\
\text { W9-060 }\end{array}$ & Grab & $\begin{array}{l}\text { Isolok sampler, } \\
\text { SP-2 }\end{array}$ & $\begin{array}{l}\text { Supply tank at } \sim 20 \text { psig, } \\
\text { Dwell time, } 7 \mathrm{~s} \\
\text { Injection time, } 1 \mathrm{~s} \\
\text { Total run time, } \sim 100 \mathrm{~h} \text {, } \\
\text { but intermittent. (See } \\
\text { logbook) } \\
\text { FLYGT MIXER } \\
\text { TOTAL RUN TIME } \\
\sim 19 \text { H PRIOR TO } \\
\text { TRANSFER }\end{array}$ & $\begin{array}{l}\text { Normal } \\
\text { system } \\
\text { operation }\end{array}$ & $\begin{array}{l}\text { Density, TS, TSS, } \\
\text { TSS50 }\end{array}$ & $\begin{array}{l}\text { Density }=1.045 \\
\text { TS }=58,200 \\
\text { TSS }=44,500 \\
\text { TSS50 }=1,340\end{array}$ \\
\hline $1-20-00$ & $\begin{array}{l}\text { GAAT- } \\
\text { W9-061 }\end{array}$ & Grab & $\begin{array}{l}\text { Isolok sampler, } \\
\text { SP-2 }\end{array}$ & $\begin{array}{l}\text { Supply tank at } \sim 20 \text { psig } \\
\text { Dwell time, } 7 \mathrm{~s} \\
\text { Injection time, } 1 \mathrm{~s} \\
\text { Total run time, } \sim 48 \mathrm{~h} \\
\text { FLYGT MIXER } \\
\quad \text { TOTAL RUN TIME } \\
\sim 0 \text { H PRIOR TO } \\
\quad \text { TRANSFER }\end{array}$ & $\begin{array}{l}\text { Normal } \\
\text { system } \\
\text { operation }\end{array}$ & $\begin{array}{l}\text { Density, pH, TS, } \\
\text { TSS, TSS50 }\end{array}$ & $\begin{array}{l}\text { Density }=1.042 \\
\text { pH }=9.75 \\
\text { TS }=50,600 \\
\text { TSS }=31,500 \\
\text { TSS50 }=950\end{array}$ \\
\hline
\end{tabular}


SAMPLE ANALYSIS DATA SUMMARY FOR ALL GAAT W-9 SLUDGE SAMPLES (Continued)

\begin{tabular}{|c|c|c|c|c|c|c|c|}
\hline \multicolumn{4}{|c|}{ Sample } & \multirow[b]{2}{*}{$\begin{array}{l}\text { PulsAir and Flygt } \\
\text { mixer status }\end{array}$} & \multirow[b]{2}{*}{$\begin{array}{c}\text { SCS } \\
\text { status }\end{array}$} & \multirow[b]{2}{*}{$\begin{array}{l}\text { Analysis } \\
\text { requested }^{b}\end{array}$} & \multirow[b]{2}{*}{$\begin{array}{c}\text { Data } \\
\text { summary }^{c} \\
\end{array}$} \\
\hline $\begin{array}{c}\text { Collection } \\
\text { date }\end{array}$ & No. & Type & Location $^{a}$ & & & & \\
\hline $2-11-00$ & $\begin{array}{l}\text { GAAT- } \\
\text { W9-062 }\end{array}$ & Grab & $\begin{array}{l}\text { Isolok sampler, } \\
\text { SP-2 }\end{array}$ & $\begin{array}{l}\text { Supply tank at } \sim 20 \text { psig } \\
\text { Dwell time, } 7 \mathrm{~s} \\
\text { Injection time, } 1 \mathrm{~s} \\
\text { Total run time, } \sim 48 \mathrm{~h} \\
\text { FLYGT MIXER } \\
\text { TOTAL RUN TIME } \\
\sim 0 \text { H PRIOR TO } \\
\text { TRANSFER }\end{array}$ & $\begin{array}{l}\text { Normal } \\
\text { system } \\
\text { operation }\end{array}$ & $\begin{array}{l}\text { Density, pH, TS, } \\
\text { TSS, TSS50 }\end{array}$ & $\begin{array}{l}\text { Density }=1.042 \\
\mathrm{pH}=9.75 \\
\mathrm{TS}=50,600 \\
\mathrm{TSS}=31,500 \\
\text { TSS50 }=950\end{array}$ \\
\hline $2-18-00$ & $\begin{array}{l}\text { GAAT } \\
\text { W9-063 }\end{array}$ & Grab & $\begin{array}{l}\text { Isolok sampler, } \\
\text { SP-2 }\end{array}$ & $\begin{array}{l}\text { Supply tank at } \sim 33 \text { psig } \\
\text { Dwell time, } 10 \mathrm{~s} \\
\text { Injection time, } 1 \mathrm{~s} \\
\text { Total run time, } \sim 96 \mathrm{~h} \\
\text { FLYGT MIXER } \\
\text { TOTAL RUN TIME } \\
\sim 48 \text { H PRIOR TO } \\
\text { TRANSFER }\end{array}$ & $\begin{array}{l}\text { Normal } \\
\text { system } \\
\text { operation }\end{array}$ & $\begin{array}{c}\text { Density, pH, TS, } \\
\text { TSS, TSS100 }\end{array}$ & $\begin{array}{l}\text { Density }=1.042 \\
\text { pH }=9.72 \\
\text { TS }=59,600 \\
\text { TSS }=35,800 \\
\text { TSS } 100=350\end{array}$ \\
\hline $3-15-00$ & $\begin{array}{l}\text { GAAT- } \\
\text { W9-064 }\end{array}$ & Grab & $\begin{array}{l}\text { Isolok sampler, } \\
\text { SP-2 }\end{array}$ & $\begin{array}{l}\text { Supply tank at } \sim 20 \text { psig } \\
\text { Dwell time, } 8 \mathrm{~s} \\
\text { Injection time, } 1 \mathrm{~s} \\
\text { Total run time, } \sim 150 \mathrm{~h} \\
\text { FLYGT MIXER } \\
\text { TOTAL RUN TIME } \\
\sim 48 \text { H PRIOR TO } \\
\text { TRANSFER }\end{array}$ & $\begin{array}{l}\text { Normal } \\
\text { system } \\
\text { operation }\end{array}$ & $\begin{array}{c}\text { Density, pH, TS, } \\
\text { TSS, TSS100 }\end{array}$ & $\begin{array}{l}\text { Density = 1.029 } \\
\mathrm{pH}=9.57 \\
\mathrm{TS}=39,200 \mathrm{mg} / \mathrm{L} \\
\mathrm{TSS}=23,000 \mathrm{mg} / \mathrm{L} \\
\mathrm{TSS} 100=130\end{array}$ \\
\hline
\end{tabular}


SAMPLE ANALYSIS DATA SUMMARY FOR ALL GAAT W-9 SLUDGE SAMPLES (Continued)

\begin{tabular}{|c|c|c|c|c|c|c|c|}
\hline \multicolumn{4}{|c|}{ Sample } & \multirow[b]{2}{*}{$\begin{array}{l}\text { PulsAir and Flygt } \\
\text { mixer status }\end{array}$} & \multirow[b]{2}{*}{$\begin{array}{l}\text { SCS } \\
\text { status }\end{array}$} & \multirow[b]{2}{*}{$\begin{array}{c}\text { Analysis } \\
\text { requested }^{b}\end{array}$} & \multirow[b]{2}{*}{$\begin{array}{c}\text { Data } \\
\text { summary }^{c}\end{array}$} \\
\hline $\begin{array}{c}\text { Collection } \\
\text { date }\end{array}$ & No. & Tyре & Location $^{a}$ & & & & \\
\hline $3-23-00$ & $\begin{array}{l}\text { GAAT } \\
\text { W9-065 }\end{array}$ & Grab & $\begin{array}{l}\text { Isolok sampler, } \\
\text { SP-2 }\end{array}$ & $\begin{array}{l}\text { Supply tank at } \sim 70 \text { psig } \\
\text { Dwell time, } 5 \mathrm{~s} \\
\text { Injection time, } 1 \mathrm{~s} \\
\text { Total run time, } \sim 348 \mathrm{~h} \\
\text { FLYGT MIXER } \\
\text { TOTAL RUN TIME } \\
\sim 48 \text { H PRIOR TO } \\
\text { TRANSFER }\end{array}$ & $\begin{array}{l}\text { Normal } \\
\text { system } \\
\text { operation, } \\
\text { new } \\
\text { restrictor } \\
\text { for PulsAir } \\
\text { System }\end{array}$ & $\begin{array}{l}\text { Density, pH, TS, } \\
\text { TSS, TSS100 }\end{array}$ & $\begin{array}{l}\text { Density }=1.039 \\
\text { pH }=9.53 \\
\text { TS }=49,900 \\
\text { TSS }=25,800 \\
\text { TSS } 100=160\end{array}$ \\
\hline $3-30-00$ & $\begin{array}{l}\text { GAAT } \\
\text { W9-066 }\end{array}$ & Grab & $\begin{array}{l}\text { Isolok sampler } \\
\text { SP-2 }\end{array}$ & $\begin{array}{l}\text { Supply tank at } \sim 65 \text { to } 73 \\
\text { psig } \\
\text { Dwell time, } 5 \mathrm{~s} \\
\text { Injection time, } 1 \mathrm{~s} \\
\text { Total run time, } \sim 185 \mathrm{~h} \\
\text { FLYGT MIXER } \\
\text { OPERATED AT } 40 \\
\text { HZ FOR A TOTAL } \\
\text { RUN TIME } \sim 24 \mathrm{H} \\
\text { PRIOR TO } \\
\text { TRANSFER }\end{array}$ & $\begin{array}{l}\text { Normal } \\
\text { system } \\
\text { operation } \\
\text { with W-9 } \\
\text { pressure at } \\
-0.4 \text { to } \\
-0.25 \text { in. } \\
\text { water }\end{array}$ & $\begin{array}{c}\text { Density, pH, TS, } \\
\text { TSS, TSS100 }\end{array}$ & $\begin{array}{l}\text { Density }=1.026 \\
\text { pH }=9.54 \\
\text { TS }=54,000 \\
\text { TSS }=30,900 \\
\text { TSS } 100=480\end{array}$ \\
\hline
\end{tabular}

${ }^{a} \mathrm{ND}=\mathrm{No}$ data

${ }^{b}$ G-beta = gross-beta radioactivity, G-alpha = gross-alpha radioactivity. TS = Total solids, TSS = Total suspended solids, TDS = Total dissolved solids.

${ }^{c}$ TDS, TS, TSS, TSS100, and TSS50 measurements are $\mathrm{mg} / \mathrm{L}$. Density measurements are $\mathrm{g} / \mathrm{mL}$. Isotopic and radioactivity measurements are $\mathrm{Bq} / \mathrm{g}$. 